\title{
Quantum Dynamical Systems
}

\author{
Claude-Alain PILLET \\ CPT-CNRS (UMR 6207) \\ Université du Sud, Toulon-Var \\ F-83957 La Garde, Cedex
}

1 Introduction $\ldots \ldots \ldots \ldots \ldots \ldots \ldots \ldots \ldots \ldots \ldots \ldots \ldots \ldots \ldots \ldots \ldots \ldots$

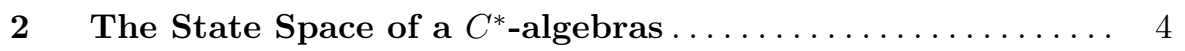

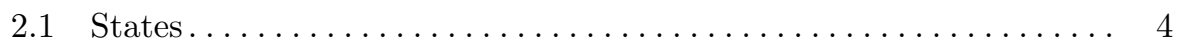

2.2 The GNS Representation $\ldots \ldots \ldots \ldots \ldots \ldots \ldots \ldots \ldots \ldots \ldots \ldots \ldots$

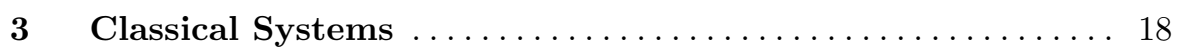

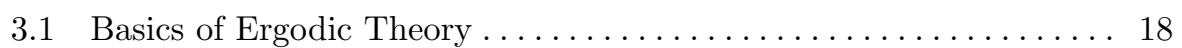

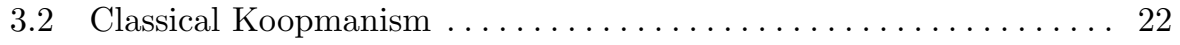

4 Quantum Systems ........................ 25

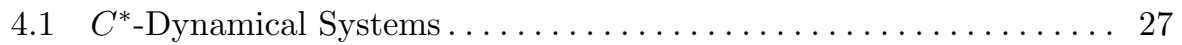

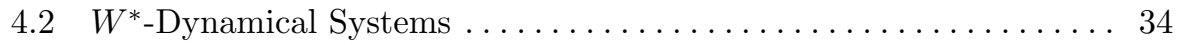

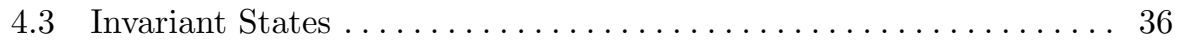

4.4 Quantum Dynamical Systems . . . . . . . . . . . . . . . . . 37

4.5 Standard Forms . . . . . . . . . . . . . . . . . . . . . . . 43

4.6 Ergodic Properties of Quantum Dynamical Systems . . . . . . . . . . 49

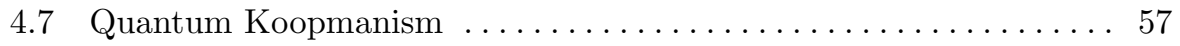

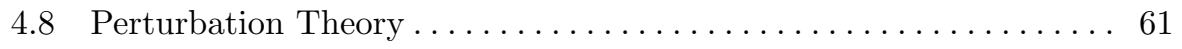

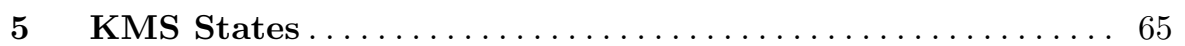

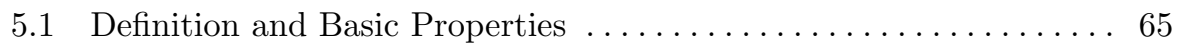

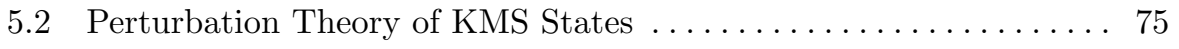

References ............................ 77

\section{Introduction}

Many problems of classical and quantum physics can be formulated in the mathematical framework of dynamical systems. Within this framework ergodic theory provides a probabilistic interpretation of dynamics which is suit- 
able to study the statistical properties of the evolution of a mechanical system over large time scales. The conceptual foundation of ergodic theory is intimately related to the birth of statistical mechanics and goes back to Boltzmann and Gibbs. It started to develop as a mathematical theory with the pioneering works of von Neumann [48] and Birkhoff [10]. It is now a beautiful cross-disciplinary part of Mathematics with numerous connections to analysis and probability, geometry, algebra, number theory, combinatorics...

The Koopman-von Neumann approach to ergodic theory [34] provides an effective way to translate ergodic properties of dynamical systems into spectral properties of some associated linear operator (which I shall call Liouvillean). The resulting spectral approach to dynamics is particularly well adapted to the study of open systems. During the last decade, this spectral approach has been successfully applied to the problem of return to equilibrium $[27,8,16$, $22,21]$ and to the construction of non-equilibrium steady states for quantum open systems [29]. The reader should consult [30] for an introduction to these problems.

The aim of this lecture is to provide a short introduction to quantum dynamical systems and their ergodic properties with particular emphasis on the quantum Koopman-von Neumann spectral theory. However, I shall not discuss the spectral analysis of the resulting Liouvillean operators. The interested reader should consult the above mentioned references. For other approaches based on scattering ideas see $[40,6,26]$.

Ergodic theory also played an important role in the development of the algebraic approach to quantum field theory and quantum statistical mechanics, mainly in connection with the analysis of symmetries. Most of the results obtained in this framework rely on some kind of asymptotic abelianness hypothesis which is often inappropriate in the context of dynamical systems. The reader should consult Chapter 4 and in particular Sections 4.3 and the corresponding notes in [11] for an introduction to the results.

I have assumed that the reader is familiar with the material covered in the first Lectures of this Volume [33, 32, 7]. Besides that, the notes are reasonably self-contained and most of the proofs are given. Numerous examples should provide the reader with a minimal toolbox to construct basic models of quantum open systems.

These notes are organized as follows.

Section 2 is an extension of Subsection 3.3 in Lecture [7]. It consists of two parts. In Subsections 2.1, I review some topological properties of von Neumann algebras. I also introduce the notions of support and central support of a normal state. Subsection 2.2 explores some elementary consequences of the GNS construction.

In Section 3, I briefly review some basic facts of the ergodic theory of classical dynamical systems. The discussion is centered around two simple properties: ergodicity and mixing. As a motivation for the transposition of these concepts to quantum mechanics I discuss the classical Koopman-von 
Neumann approach in Subsection 3.2. General references for Section 3 are [15], [35], [49] and [5].

The main part of these notes is contained in Section 4 which deals with the ergodic theory of quantum systems. The basic concepts of the algebraic theory of quantum dynamics $-C^{*}$ - and $W^{*}$-dynamical systems and their invariant states - are introduced in Subsections 4.1-4.3.

In Subsection 4.4, I define a more general notion of quantum dynamical system. The GNS construction provides an efficient way to bring such systems into normal form. This normal form plays an essential role in quantum ergodic theory. In particular it allows to define a Liouvillean which will be the central object of the quantum Koopman-von Neumann theory. In Subsection 4.5, I introduce the related notion of standard form of a quantum dynamical system.

The ergodic properties - ergodicity and mixing - of quantum dynamical systems are defined and studied in Subsection 4.6. The quantum Koopmanvon Neumann spectral theory is developed in Subsection 4.7.

In many physical applications, and in particular in simple models of open systems, the dynamics is constructed by coupling elementary subsystems. Perturbation theory provides a powerful tool to analyze such models. In Subsection 4.8, I discuss a simple adaptation of the Dyson-Schwinger-Tomonaga time dependent perturbation theory - which played an important role in the early development of quantum electrodynamics - to $C^{*}$ - and $W^{*}$-dynamical systems.

General references for the material in Section 4 are Chapters 2.5, 3.1 and 3.2 in [11] as well as [47], [46] and [44]. More examples of dynamical systems can be found in [9].

Among the invariant states of a $C^{*}$ - or $W^{*}$-dynamical system, the KMS states introduced in Section 5 form a distinguished class, from the physical as well as from the mathematical point of view. On the physical side, KMS states play the role of thermodynamical equilibrium states. As such, they are basic building blocks for an important class of models of open quantum systems where the reservoirs are at thermal equilibrium. On the mathematical side, KMS states appear naturally in the modular structure associated with faithful normal states (or more generally semi-finite weight) on von Neumann algebras. They are thus intimately connected with their mathematical structure. This tight relation between dynamics and the structure of the observable algebra is one of the magical feature of quantum mechanics which has no classical counterpart.

In Subsection 5.2, I discuss the perturbation theory of KMS states. For bounded perturbations, the theory is due to Araki [2] and [4] (see also Section 5.4.1 in [12]). Extensions to unbounded perturbations have been developed in $[43],[19]$ and [17]. This subject being very technical, I only give some plausibility argument and state the main results without proofs.

Acknowledgments. I wish to thank Jan Dereziński and Vojkan Jakšić for fruitful discussions related to the material covered by these notes. I am par- 
ticularly grateful to Stephan De Bièvre for his constructive comments on an early version of the manuscript.

\section{The State Space of a $C^{*}$-algebras}

This section is a complement to Lecture [7] and contains a few additions that are needed to develop the ergodic theory of quantum dynamical systems. It consists of two parts.

In the first part I present the basic properties of normal states on a von Neumann algebra $\mathfrak{M}$. In particular I discuss the connection between the $\sigma$ weak topology on $\mathfrak{M}$ and its algebraic structure. I also introduce the very useful concepts of support and central support of a normal state.

The second part of the section deals with the GNS construction and its consequences on the structure of the state space of a subsection $C^{*}$-algebra: enveloping von Neumann algebra and folium of a state, relative normality and orthogonality of states. I also discuss the special features of the GNS representation associated with a normal state.

The material covered by this section is standard and the reader already familiar with the above concepts may skip it.

\section{Warning: All $C^{*}$-algebras in this lecture have a unit $I$.}

\subsection{States}

A linear functional $\omega$ on a $C^{*}$-algebra $\mathfrak{A}$ is positive if $\omega\left(A^{*} A\right) \geq 0$ for all $A \in \mathfrak{A}$. Such a functional is automatically continuous and $\|\omega\|=\omega(I)$ (see Proposition 5 in Lecture [7]). If $\mu, \nu$ are two positive linear functionals such that $\nu-\mu$ is a positive linear functional then we write $\mu \leq \nu$.

A state is a normalized $(\|\omega\|=1)$ positive linear functional. A state $\omega$ is faithful if $\omega\left(A^{*} A\right)=0$ implies $A=0$.

Denote by $\mathfrak{A}_{1}^{\star}$ the unit ball of the Banach space dual $\mathfrak{A}^{\star}$. By the BanachAlaoglu theorem, $\mathfrak{A}_{1}^{\star}$ is compact in the weak- $\star$ topology. The set of all states on $\mathfrak{A}$ is given by

$$
E(\mathfrak{A})=\left\{\omega \in \mathfrak{A}_{1}^{\star} \mid \omega\left(A^{*} A\right) \geqslant 0 \text { for all } A \in \mathfrak{A}\right\},
$$

and it immediately follows that it is a weak-^ compact, convex subset of $\mathfrak{A}^{\star}$.

\section{Normal States}

Recall (Subsection 3.1 in Lecture [7]) that the $\sigma$-weak topology on a von Neumann algebra $\mathfrak{M} \subset \mathfrak{B}(\mathcal{H})$ is the locally convex topology generated by the semi-norms 


$$
A \mapsto \sum_{n \in \mathbb{N}}\left|\left(\psi_{n}, A \phi_{n}\right)\right|,
$$

for sequences $\psi_{n}, \phi_{n} \in \mathcal{H}$ such that $\sum_{n}\left\|\psi_{n}\right\|^{2}<\infty$ and $\sum_{n}\left\|\phi_{n}\right\|^{2}<\infty$. The $\sigma$-strong and $\sigma$-strong* topologies are defined similarly by the semi-norms

$$
A \mapsto\left(\sum_{n \in \mathbb{N}}\left\|A \psi_{n}\right\|^{2}\right)^{1 / 2}, \quad A \mapsto\left(\sum_{n \in \mathbb{N}}\left\|A \psi_{n}\right\|^{2}+\sum_{n \in \mathbb{N}}\left\|A^{*} \psi_{n}\right\|^{2}\right)^{1 / 2},
$$

with $\sum_{n}\left\|\psi_{n}\right\|^{2}<\infty$. Note that, except when $\mathcal{H}$ is finite dimensional, these topologies are not first countable. Therefore, the use of nets (directed sets) is mandatory.

As a Banach space, the von Neumann algebra $\mathfrak{M}$ is the dual of the space $\mathfrak{M}_{\star}$ of all $\sigma$-weakly continuous linear functionals on $\mathfrak{M}$. In particular, the predual $\mathfrak{M}_{\star}$ is a norm-closed subspace of the dual $\mathfrak{M}^{\star}=\left(\mathfrak{M}_{\star}\right)^{\star \star}$.

Exercise 1. Show that the $\sigma$-strong* topology is stronger than the $\sigma$-strong topology which is stronger than the $\sigma$-weak topology. Show also that the $\sigma$-strong (resp. $\sigma$-weak) topology is stronger than the strong (resp. weak) topology and that these two topologies coincide on norm bounded subsets.

Exercise 2. Adapt the proof of Proposition 8.ii in Lecture [7] to show that a linear functional $\omega$ on $\mathfrak{M}$ is $\sigma$-weakly continuous if and only if it is $\sigma$-strongly continuous. Using Corollary 2 in Lecture [7] and the Hahn-Banach theorem show that $\omega$ is $\sigma$-weakly continuous if and only if there exists a trace class operator $T$ on $\mathcal{H}$ such that $\omega(A)=\operatorname{Tr}(T A)$ for all $A \in \mathfrak{M}$.

The von Neumann density theorem (Theorem 13 in Lecture [7]) asserts that a $*$-subalgebra $\mathcal{D} \subset \mathfrak{B}(\mathcal{H})$ containing $I$ is dense in $\mathcal{D}^{\prime \prime}$ in the weak and strong topologies. In fact one can prove more (see for example Corollary 2.4.15 in [11]).

Theorem 1. (Von Neumann density theorem) $A *$-subalgebra $\mathcal{D} \subset \mathfrak{B}(\mathcal{H})$ containing $I$ is $\sigma$-strongly* dense in $\mathcal{D}^{\prime \prime}$.

Thus, any element $A$ of the von Neumann algebra generated by $\mathfrak{D}$ is the $\sigma$-strong* limit of a net $A_{\alpha}$ in $\mathfrak{D}$. By Exercise 1 the net $A_{\alpha}$ also approximates $A$ in the $\sigma$-strong, $\sigma$-weak, strong and weak topologies. In particular, $\mathfrak{D}^{\prime \prime}$ coincides with the closure of $\mathfrak{D}$ in all these topologies. It is often useful to approximate $A$ by a bounded net in $\mathfrak{D}$. That this is also possible is a simple consequence of the following theorem (see Theorem 2.4.16 in [11]).

Theorem 2. (Kaplansky density theorem) Let $\mathcal{D} \subset \mathfrak{B}(\mathcal{H})$ be a $*$-subalgebra and denote by $\overline{\mathfrak{D}}$ its weak closure. Then $\mathfrak{D}_{r} \equiv\{A \in \mathfrak{D} \mid\|A\| \leq r\}$ is $\sigma$-strongly* dense in $\overline{\mathfrak{D}}_{r} \equiv\{A \in \overline{\mathfrak{D}} \mid\|A\| \leq r\}$ for any $r>0$. 
Recall also that a self-adjoint element $A$ of a $C^{*}$-algebra $\mathfrak{A}$ is positive if its spectrum is a subset of $[0, \infty[$ (see Theorem 5 in Lecture [7]). This definition induces a partial order on the set of self-adjoint elements of $\mathfrak{A}: A \leq B$ if and only if $B-A$ is positive. Moreover, one writes $A<B$ if $A \leq B$ and $A \neq B$. The relation $\leq$ is clearly a purely algebraic concept, i.e., it is independent on the action of $\mathfrak{A}$ on some Hilbert space. However, if $\mathfrak{A}$ acts on a Hilbert space $\mathcal{H}$ then its positive elements are characterized by the fact that $(\psi, A \psi) \geq 0$ for all $\psi$ in a dense subspace of $\mathcal{H}$. In particular, $A \leq B$ if and only if $(\psi, A \psi) \leq(\psi, B \psi)$ for all $\psi$ in such a subspace.

Let $A_{\alpha}$ be a bounded increasing net of self-adjoint elements of $\mathfrak{B}(\mathcal{H})$, i.e., such that $A_{\alpha} \geq A_{\beta}$ for $\alpha \succ \beta$ and $\sup _{\alpha}\left\|A_{\alpha}\right\|<\infty$. Then, for any $\psi \in \mathcal{H}$, one has $\sup _{\alpha}\left(\bar{\psi}, A_{\alpha} \psi\right)<\infty$ and since a self-adjoint element $A$ of $\mathfrak{B}(\mathcal{H})$ is completely determined by its quadratic form $\psi \mapsto(\psi, A \psi)$, there exists a unique $A \in \mathfrak{B}(\mathcal{H})$ such that

$$
(\psi, A \psi) \equiv \lim _{\alpha}\left(\psi, A_{\alpha} \psi\right)=\sup _{\alpha}\left(\psi, A_{\alpha} \psi\right) .
$$

It follows immediately from this definition that $A=\sup _{\alpha} A_{\alpha}$. Since one has $0 \leq A-A_{\alpha} \leq A$, the estimate

$$
\begin{aligned}
\left\|\left(A-A_{\alpha}\right) \psi\right\|^{2} & =\left(\psi,\left(A-A_{\alpha}\right)^{2} \psi\right) \\
& \leq\left\|A-A_{\alpha}\right\|\left\|\left(A-A_{\alpha}\right)^{1 / 2} \psi\right\|^{2} \\
& \leq\|A\|\left(\psi,\left(A-A_{\alpha}\right) \psi\right),
\end{aligned}
$$

further shows that $A_{\alpha}$ converges strongly to $A$. Moreover, since the net $A_{\alpha}$ is bounded, one also has $\lim _{\alpha} A_{\alpha}=A$ in the $\sigma$-strong and $\sigma$-weak topologies (Exercise 1). In particular, if $\mathfrak{M} \subset \mathfrak{B}(\mathcal{H})$ is a von Neumann algebra and $A_{\alpha} \in \mathfrak{M}$, then $A \in \mathfrak{M}$. Finally, we note that if $B \in \mathfrak{M}$ then

$$
\sup _{\alpha}\left(B^{*} A_{\alpha} B\right)=B^{*}\left(\sup _{\alpha} A_{\alpha}\right) B .
$$

Definition 1. A positive linear functional $\omega$ on a von Neumann algebra $\mathfrak{M}$ is called normal if, for all bounded increasing net $A_{\alpha}$ of self-adjoint elements of $\mathfrak{M}$, one has

$$
\omega\left(\sup _{\alpha} A_{\alpha}\right)=\sup _{\alpha} \omega\left(A_{\alpha}\right) .
$$

In particular, a normal state is a normalized, normal, positive linear functional.

Remark 1. This definition differs from the one given in Section 3.3 of Lecture [7]. However, Theorem 3 below shows that these two definitions are equivalent.

Note that the concept of normality only depends on the partial order relation $\leq$ and hence on the algebraic structure of $\mathfrak{M}$. Since by Exercise 2 any $\sigma$-weakly continuous linear functional on $\mathfrak{M}$ is of the form $A \mapsto \operatorname{Tr}(T A)$ for some trace class operator $T$, it is a finite linear combination of positive, 
$\sigma$-weakly continuous linear functionals (because $T$ is a linear combination of 4 positive trace class operators). Thus, the following theorem characterizes the $\sigma$-weak topology on $\mathfrak{M}$ in a purely algebraic way.

Theorem 3. A positive linear functional on a von Neumann algebra is normal if and only if it is $\sigma$-weakly continuous.

Proof. If $\omega$ is a $\sigma$-weakly continuous positive linear functional on the von Neumann algebra $\mathfrak{M}$ and $A_{\alpha}$ a bounded increasing net of self-adjoint elements of $\mathfrak{M}$ one has, in the $\sigma$-weak topology,

$$
\omega\left(\sup _{\alpha} A_{\alpha}\right)=\omega\left(\lim _{\alpha} A_{\alpha}\right)=\lim _{\alpha} \omega\left(A_{\alpha}\right)=\sup _{\alpha} \omega\left(A_{\alpha}\right) .
$$

Hence, $\omega$ is normal.

To prove the reverse statement let $\omega$ be a normal positive linear functional and consider the set

$$
\mathcal{A} \equiv\left\{A \in \mathfrak{M} \mid 0 \leq A \leq I, \omega_{A} \in \mathfrak{M}_{\star}\right\},
$$

where $\omega_{A}(X) \equiv \omega(X A)$. If $0 \leq B \leq A \leq I$ then the Cauchy-Schwarz inequality,

$$
\begin{aligned}
\left|\omega_{A}(X)-\omega_{B}(X)\right|^{2} & =|\omega(X(A-B))|^{2} \\
& \leq \omega\left(X(A-B) X^{*}\right) \omega(A-B) \\
& \leq\|X\|^{2} \omega(A-B),
\end{aligned}
$$

yields that

$$
\left\|\omega_{A}-\omega_{B}\right\|^{2} \leq \omega(A)-\omega(B) .
$$

Let $A_{\alpha}$ be an increasing net in $\mathcal{A}$ and set $A \equiv \sup _{\alpha} A_{\alpha}$. One clearly has $0 \leq A_{\alpha} \leq A \leq I$ and since $\omega$ is normal Equ. (2) shows that $\omega_{A_{\alpha}}$ converges in norm to $\omega_{A} \cdot \mathfrak{M}_{\star}$ being a norm-closed subspace of $\mathfrak{M}^{\star}$ one has $\omega_{A} \in \mathfrak{M}_{\star}$ and we conclude that $A \in \mathcal{A}$. Thus, $\mathcal{A}$ is inductively ordered and by Zorn's lemma there exists a maximal element $N \in \mathcal{A}$. We set $M \equiv I-N$ and note that if $\omega(M)=0$ then Equ. (2) shows that $\omega=\omega_{N} \in \mathfrak{M}_{\star}$.

To conclude the proof we assume that $\omega(M)>0$ and show that this leads to a contradiction. Since $M>0$ we can pick $\psi \in \mathcal{H}$ such that $\omega(M)<$ $(\psi, M \psi)$. Consider an increasing net $B_{\alpha}$ in the set

$$
\mathcal{B} \equiv\{B \in \mathfrak{M} \mid 0 \leq B \leq M, \omega(B) \geq(\psi, B \psi)\},
$$

and let $B \equiv \sup _{\alpha} B_{\alpha}$. Then $0 \leq B_{\alpha} \leq B \leq M$ and since $\omega$ is normal

$$
\omega(B)=\sup _{\alpha} \omega\left(B_{\alpha}\right) \geq \sup _{\alpha}\left(\psi, B_{\alpha} \psi\right)=(\psi, B \psi),
$$

shows that $B \in \mathcal{B}$. Hence $\mathcal{B}$ is inductively ordered. Let $S$ be a maximal element of $\mathcal{B}$. Remark that $M \notin \mathcal{B}$ since $\omega(M)<(\psi, M \psi)$. This means that 


$$
T \equiv M-S>0 .
$$

Next we note that if $0 \leq B \leq T$ and $\omega(B) \geq(\psi, B \psi)$ then $B+S \in \mathcal{B}$ and the maximality of $S$ yields that $B=0$. It follows that $0 \leq B \leq T$ implies $\omega(B) \leq(\psi, B \psi)$. Since for any $B \in \mathfrak{M}$ such that $\|B\| \leq 1$ one has

$$
T B^{*} B T \leq T^{2} \leq T,
$$

we can conclude that

$$
\omega\left(T B^{*} B T\right) \leq\left(\psi, T B^{*} B T \psi\right)=\|B T \psi\|^{2} .
$$

By Cauchy-Schwarz inequality we further get

$$
\left|\omega_{T}(B)\right|^{2}=|\omega(I B T)|^{2} \leq \omega(I) \omega\left(T B^{*} B T\right) \leq\|B T \psi\|^{2} .
$$

This inequality extends by homogeneity to all $B \in \mathfrak{M}$ and shows that $\omega_{T}$ is $\sigma$-strongly continuous and hence, by Exercise $2, \sigma$-weakly continuous. Finally, we note that $\omega_{N+T}=\omega_{N}+\omega_{T} \in \mathfrak{M}_{\star}$ and by Equ. (3),

$$
N<N+T=N+(M-S)=I-S \leq I,
$$

a contradiction to the maximality of $N$.

Thus, the set of normal states on a von Neumann algebra $\mathfrak{M}$ coincides with the set of $\sigma$-weakly continuous states and with the set of $\sigma$-strongly continuous states. It is given by

$$
N(\mathfrak{M})=\mathfrak{M}_{\star} \cap E(\mathfrak{M}) \subset E(\mathfrak{M}),
$$

and is clearly a norm closed subset of $E(\mathfrak{M})$. If $\mathfrak{M}$ acts on the Hilbert space $\mathcal{H}$ then, according to Exercise 2, a normal state $\omega$ on $\mathfrak{M}$ is described by a density matrix, i.e., a non-negative trace class operator $\rho$ on $\mathcal{H}$ such that $\operatorname{Tr} \rho=1$ and $\omega(A)=\operatorname{Tr}(\rho A)$.

Lemma 1. Let $\mathfrak{M} \subset \mathfrak{B}(\mathcal{H})$ be a von Neumann algebra. The set of vector states $V(\mathfrak{M}) \equiv\{(\Psi,(\cdot) \Psi) \mid \Psi \in \mathcal{H},\|\Psi\|=1\}$ is total in $N(\mathfrak{M})$ i.e., finite convex linear combinations of elements of $V(\mathfrak{M})$ are norm dense in $N(\mathfrak{M})$.

Proof. We first note that if $\mu, \nu \in N(\mathfrak{M})$ are given by density matrices $\rho, \sigma$ then

$$
|\mu(A)-\nu(A)|=|\operatorname{Tr}((\rho-\sigma) A)| \leq\|\rho-\sigma\|_{1}\|A\|,
$$

where $\|T\|_{1} \equiv \operatorname{Tr}\left(T^{*} T\right)^{1 / 2}$ denotes the trace norm. Hence $\|\mu-\nu\| \leq\|\rho-\sigma\|_{1}$. Let $\mu \in N(\mathfrak{M})$ and $\rho$ a corresponding density matrix. Denote by

$$
\rho=\sum_{n} p_{n} \psi_{n}\left(\psi_{n}, \cdot\right)
$$


its spectral decomposition, i.e., $\left(\psi_{n}, \psi_{k}\right)=\delta_{n, k}, 0<p_{n} \leq 1, \sum_{n} p_{n}=1$. From the trace norm estimate

$$
\left\|\rho-\sum_{n=1}^{N-1} p_{n} \psi_{n}\left(\psi_{n}, \cdot\right)\right\|_{1}=\left\|\sum_{n \geq N} p_{n} \psi_{n}\left(\psi_{n}, \cdot\right)\right\|_{1}=\sum_{n \geq N} p_{n} \equiv q_{N},
$$

it follows that

$$
\left\|\mu-\left(\sum_{n=1}^{N-1} p_{n} \mu_{n}+q_{N} \mu_{N}\right)\right\| \leq 2 q_{N}
$$

where $\mu_{n}=\left(\psi_{n},(\cdot) \psi_{n}\right) \in V(\mathfrak{M})$. Since $\lim _{N} q_{N}=0$ we conclude that finite convex linear combinations of vector states are norm dense in $N(\mathfrak{M})$.

Exercise 3. (Complement to Lemma 1) Let $\mathcal{M} \subset \mathcal{H}$ be a dense subspace. Show that the set of vector states $V_{\mathcal{D}}(\mathfrak{M}) \equiv\{(\Psi,(\cdot) \Psi) \mid \Psi \in \mathcal{D},\|\Psi\|=1\}$ is total in $N(\mathfrak{M})$.

Exercise 4. Show that a net $A_{\alpha}$ in a von Neumann algebra $\mathfrak{M}$ converges $\sigma$-weakly (resp. $\sigma$-strongly) to 0 if and only if, for all $\omega \in N(\mathfrak{M})$ one has $\lim _{\alpha} \omega\left(A_{\alpha}\right)=0\left(\right.$ resp. $\left.\lim _{\alpha} \omega\left(A_{\alpha}^{*} A_{\alpha}\right)=0\right)$.

Lemma 2. Let $\mathfrak{M}, \mathfrak{N}$ be von Neumann algebras. $A *$-morphism $\phi: \mathfrak{M} \rightarrow \mathfrak{N}$ is $\sigma$-weakly continuous if and only if it is $\sigma$-strongly continuous.

Proof. Suppose that $\phi$ is $\sigma$-weakly continuous and that the net $A_{\alpha}$ converges $\sigma$-strongly to 0 . By Exercise $4, A_{\alpha}^{*} A_{\alpha}$ converges $\sigma$-weakly to 0 . It follows that $\phi\left(A_{\alpha}\right)^{*} \phi\left(A_{\alpha}\right)=\phi\left(A_{\alpha}^{*} A_{\alpha}\right)$ converges $\sigma$-weakly to zero and hence, by Exercise 4 again, that $\phi\left(A_{\alpha}\right)$ converges $\sigma$-strongly to 0 .

Suppose now that $\phi$ is $\sigma$-strongly continuous. Since any $\omega \in N(\mathfrak{N})$ is $\sigma$ strongly continuous, so is the state $\omega \circ \phi$. This means that $\omega \circ \phi$ is $\sigma$-weakly continuous for all $\omega \in N(\mathfrak{N})$ and hence that $\phi$ itself is $\sigma$-weakly continuous.

Corollary 1. A *-isomorphism between two von Neumann algebras is $\sigma$ weakly and $\sigma$-strongly continuous.

Proof. Let $\mathfrak{M}, \mathfrak{N}$ be von Neumann algebras and $\phi: \mathfrak{M} \rightarrow \mathfrak{N}$ a $*$-isomorphism. If $A_{\alpha}$ is a bounded increasing net of self-adjoint elements in $\mathfrak{M}$ then so is $\phi\left(A_{\alpha}\right)$ in $\mathfrak{N}$. Set $A \equiv \sup _{\alpha} A_{\alpha}$. Since $\phi$ preserves positivity one has $\phi\left(A_{\alpha}\right) \leq \phi(A)$ and hence $\sup _{\alpha} \phi\left(A_{\alpha}\right) \leq \phi(A)$. Moreover, since $\phi$ is surjective there exists $B \in \mathfrak{M}$ such that $\sup _{\alpha} \phi\left(A_{\alpha}\right)=\phi(B)$ and

$$
\phi\left(A_{\alpha}\right) \leq \phi(B) \leq \phi(A),
$$

holds for all $\alpha$. These inequalities and the injectivity of $\phi$ further yield

$$
A_{\alpha} \leq B \leq A,
$$


for all $\alpha$. Thus, we conclude that $B=A$, that is,

$$
\sup _{\alpha} \phi\left(A_{\alpha}\right)=\phi\left(\sup _{\alpha} A_{\alpha}\right) .
$$

By Theorem 3, any $\omega \in N(\mathfrak{N})$ is normal and Equ. (4) yields

$$
\sup _{\alpha} \omega\left(\phi\left(A_{\alpha}\right)\right)=\omega\left(\sup _{\alpha} \phi\left(A_{\alpha}\right)\right)=\omega\left(\phi\left(\sup _{\alpha} A_{\alpha}\right)\right),
$$

which shows that $\omega \circ \phi$ is normal and hence $\sigma$-weakly continuous. It follows that $\phi$ itself is $\sigma$-weakly continuous and, by Lemma $2, \sigma$-strongly continuous.

\section{Functional Calculus}

Let $\mathfrak{A}$ be a $C^{*}$-algebra and $A \in \mathfrak{A}$ a self-adjoint element. By Theorem 4 in Lecture [7] there is a unique $*$-morphism $\pi_{A}: C(\sigma(A)) \rightarrow \mathfrak{A}$ such that $\pi_{A}(f)=A$ if $f(x)=x$. Accordingly, if $f$ is continuous we write $f(A) \equiv \pi_{A}(f)$.

When dealing with a von Neumann algebra $\mathfrak{M} \subset \mathfrak{B}(\mathcal{H})$, it is necessary to extend this morphism to a larger class of functions. This can be done with the help of Theorem 7 and Remark 10 in Lecture [32]. Let $A \in \mathfrak{B}(\mathcal{H})$ be self-adjoint and denote by $\mathcal{B}(\mathbb{R})$ the $*$-algebra of bounded Borel functions on $\mathbb{R}$. Then there exists a unique $*$-morphism $\Pi_{A}: \mathcal{B}(\mathbb{R}) \rightarrow \mathfrak{B}(\mathcal{H})$ such that

(i) $\Pi_{A}(f)=f(A)$ if $f \in C(\sigma(A))$.

(ii) If $f, f_{n} \in \mathcal{B}(\mathbb{R})$ are such that $\lim _{n} f_{n}(x)=f(x)$ for all $x \in \mathbb{R}$ and $\sup _{n, x \in \mathbb{R}}\left|f_{n}(x)\right|<\infty$ then $\Pi_{A}\left(f_{n}\right) \rightarrow \Pi_{A}(f)$ strongly.

Again we write $f(A) \equiv \Pi_{A}(f)$ for $f \in \mathcal{B}(\mathbb{R})$. Thus, if $A \in \mathfrak{M}$ is self-adjoint then $f(A) \in \mathfrak{M}$ for any continuous function $f$. More generally, assume that $f \in \mathcal{B}(\mathcal{R})$ can be approximated by a sequence $f_{n}$ of continuous functions such that (ii) holds. Since $\mathfrak{M}$ is strongly closed it follows that $f(A) \in \mathfrak{M}$.

In particular, if $\chi_{I}$ denotes the characteristic function of an interval $I \subset \mathbb{R}$ then $\chi_{I}(A)$ is the spectral projection of $A$ for the interval $I$ and one has $\chi_{I}(A) \in \mathfrak{M}$.

\section{The Support of a Normal State}

Exercise 5. Let $P_{\alpha}$ be an increasing net of orthogonal projections of the Hilbert space $\mathcal{H}$. Denote by $P$ the orthogonal projection on the smallest closed subspace of $\mathcal{H}$ containing all the subspaces $\operatorname{Ran} P_{\alpha}$. Show that

$$
\mathrm{s}-\lim _{\alpha} P_{\alpha}=\sup _{\alpha} P_{\alpha}=P .
$$

Exercise 6. Let $P$ and $Q$ be two orthogonal projections on the Hilbert space $\mathcal{H}$. Denote by $P \wedge Q$ the orthogonal projection on $\operatorname{Ran} P \cap \operatorname{Ran} Q$ and by $P \vee Q$ the orthogonal projection on $\operatorname{Ran} P+\operatorname{Ran} Q$. 
$i$. Show that $I-P \vee Q=(I-P) \wedge(I-Q)$.

ii. Show that $P \vee Q \leq P+Q \leq I+P \wedge Q$.

iii. Set $T \equiv P Q P$ and show that $\operatorname{Ran} P \cap \operatorname{Ran} Q=\operatorname{Ker}(I-T)$. Mimic the proof of Theorem 7 to show that

$$
P \wedge Q=\mathrm{s}-\lim _{n \rightarrow \infty} T^{n} .
$$

$i v$. Show that if $\mathfrak{M}$ is a von Neumann algebra on $\mathcal{H}$ and if $P, Q \in \mathfrak{M}$ then $P \wedge Q \in \mathfrak{M}$ and $P \vee Q \in \mathfrak{M}$.

$v$. Show that if $\omega \in E(\mathfrak{M})$ then $\omega(P \vee Q)=0$ if and only if $\omega(P)=\omega(Q)=0$ and $\omega(P \wedge Q)=1$ if and only if $\omega(P)=\omega(Q)=1$.

Let $\omega$ be a normal state on the von Neumann algebra $\mathfrak{M}$. We denote by $\mathfrak{M}^{P}$ the set of orthogonal projections in $\mathfrak{M}$. Exercise 5 shows that the nonempty set $\mathfrak{P}_{\omega} \equiv\left\{P \in \mathfrak{M}^{P} \mid \omega(P)=0\right\}$ is inductively ordered: any increasing net $P_{\alpha}$ in $\mathfrak{P}_{\omega}$ has a least upper bound

$$
\sup _{\alpha} P_{\alpha}=\mathrm{s}-\lim _{\alpha} P_{\alpha} \in \mathfrak{P}_{\omega} .
$$

By Zorn's lemma, $\mathfrak{P}_{\omega}$ has a maximal element $\bar{P}_{\omega}$. For any $P \in \mathfrak{P}_{\omega}$ one has $P \vee \bar{P}_{\omega} \geq \bar{P}_{\omega}$ and, by exercise $6, P \vee \bar{P}_{\omega} \in \mathfrak{P}_{\omega}$. The maximality of $\bar{P}_{\omega}$ yields $P \vee \bar{P}_{\omega}=\bar{P}_{\omega}$ from which we can conclude that $P \leq \bar{P}_{\omega}$. Thus, one has $\bar{P}_{\omega}=\sup \mathfrak{P}_{\omega}$. The complementary projection

$$
s_{\omega} \equiv I-\bar{P}_{\omega}=\inf \left\{P \in \mathfrak{M}^{P} \mid \omega(P)=1\right\},
$$

is called the support of $\omega$. For any normal state $\omega$ and any $A \in \mathfrak{M}$ one has, by the Cauchy-Schwarz inequality

$$
\left|\omega\left(A\left(I-s_{\omega}\right)\right)\right| \leq \omega\left(A A^{*}\right)^{1 / 2} \omega\left(I-s_{\omega}\right)^{1 / 2}=0,
$$

from which we conclude that $\omega(A)=\omega\left(A s_{\omega}\right)=\omega\left(s_{\omega} A\right)$.

Exercise 7. Show that $\omega\left(A^{*} A\right)=0$ if and only if $A s_{\omega}=0$. Conclude that the state $\omega$ is faithful if and only if $s_{\omega}=I$.

Hint: if $A s_{\omega} \neq 0$ there exists $\epsilon>0$ and a non-zero $P_{\epsilon} \in \mathfrak{M}^{P}$ such that $s A^{*} A s \geq \epsilon P_{\epsilon}$ and $P_{\epsilon}=P_{\epsilon} s_{\omega}$.

Exercise 8. Let $\mathfrak{M} \subset \mathfrak{B}(\mathcal{H})$ be a von Neumann algebra. If $\mathcal{K} \subset \mathcal{H}$ is a vector subspace, denote by $[\mathcal{K}]$ the orthogonal projection on its closure $\overline{\mathcal{K}}$. Use Proposition 10 in Lecture [7] to show that, for any subset $\mathcal{M} \subset \mathcal{H}$, $\left[\mathfrak{M}^{\prime} \mathcal{M}\right] \in \mathfrak{M}$.

Lemma 3. Let $\mathfrak{M} \subset \mathfrak{B}(\mathcal{H})$ be a von Neumann algebra. The support of the state $\omega \in N(\mathfrak{M})$ is given by

$$
s_{\omega}=\left[\mathfrak{M}^{\prime} \operatorname{Ran} \rho\right],
$$


where $\rho$ is any density matrix on $\mathcal{H}$ such that $\omega(A)=\operatorname{Tr}(\rho A)$ for all $A \in \mathfrak{M}$. In particular, the support of the vector state $\omega_{\Phi}(A)=(\Phi, A \Phi)$ is given by

$$
s_{\omega_{\Phi}}=\left[\mathfrak{M}^{\prime} \Phi\right] .
$$

$\omega_{\Phi}$ is faithful if and only if $\Phi$ is cyclic for $\mathfrak{M}^{\prime}$.

Proof. Set $P=\left[\mathfrak{M}^{\prime} \operatorname{Ran} \rho\right]$ and note that $P \in \mathfrak{M}$ by Exercise 8 . On the one hand $I \in \mathfrak{M}^{\prime}$ implies $\operatorname{Ran} \rho \subset \mathfrak{M}^{\prime} \operatorname{Ran} \rho$ and hence $P \operatorname{Ran} \rho=\operatorname{Ran} \rho$. Thus, $P \rho=\rho$ and $\omega(P)=\operatorname{Tr}(P \rho)=\operatorname{Tr}(\rho)=1$, from which we conclude that $P \geq s_{\omega}$. On the other hand,

$$
0=\omega\left(I-s_{\omega}\right)=\operatorname{Tr}\left(\rho\left(I-s_{\omega}\right)\right)=\operatorname{Tr}\left(\rho^{1 / 2}\left(I-s_{\omega}\right) \rho^{1 / 2}\right)=\left\|\left(I-s_{\omega}\right) \rho^{1 / 2}\right\|_{2}^{2},
$$

yields $\left(I-s_{\omega}\right) \rho=0$. It follows that $s_{\omega} \operatorname{Ran} \rho=\operatorname{Ran} \rho$ and

$$
s_{\omega} \mathfrak{M}^{\prime} \operatorname{Ran} \rho=\mathfrak{M}^{\prime} s_{\omega} \operatorname{Ran} \rho=\mathfrak{M}^{\prime} \operatorname{Ran} \rho,
$$

implies $s_{\omega} P=P$, that is $P \leq s_{\omega}$.

\section{The Central Support of a Normal State}

The center of a von Neumann algebra $\mathfrak{M}$ is the Abelian von Neumann subalgebra $\mathfrak{Z}(\mathfrak{M}) \equiv \mathfrak{M} \cap \mathfrak{M}^{\prime}$. One easily sees that $\mathfrak{Z}(\mathfrak{M})=\left(\mathfrak{M} \cup \mathfrak{M}^{\prime}\right)^{\prime}$ so that $\mathfrak{Z}(\mathfrak{M})^{\prime}=\mathfrak{M} \vee \mathfrak{M}^{\prime}$ is the smallest von Neumann algebra containing $\mathfrak{M}$ and $\mathfrak{M}^{\prime}$. The elementary proof of the following lemma is left to the reader.

Lemma 4. Assume that $\mathfrak{M}$ and $\mathfrak{N}$ are two von Neumann algebras and let $\phi: \mathfrak{M} \rightarrow \mathfrak{N}$ be a $*$-morphism.

(i) If $\phi$ is surjective then $\phi(\mathfrak{Z}(\mathfrak{M})) \subset \mathfrak{Z}(\mathfrak{N})$.

(ii) If $\phi$ is injective then $\phi^{-1}(\mathfrak{Z}(\mathfrak{N})) \subset \mathfrak{Z}(\mathfrak{M})$.

(iii) If $\phi$ is bijective then $\phi(\mathfrak{Z}(\mathfrak{M}))=\mathfrak{Z}(\mathfrak{N})$.

$\mathfrak{M}$ is a factor if $\mathfrak{Z}(\mathfrak{M})=\mathbb{C} I$ or equivalently $\mathfrak{M} \vee \mathfrak{M}^{\prime}=\mathfrak{B}(\mathcal{H})$.

The central support of a normal state $\omega$ on $\mathfrak{M}$ is the support of its restriction to the center of $\mathfrak{M}$,

$$
z_{\omega} \equiv \inf \left\{P \in \mathfrak{Z}(\mathfrak{M}) \cap \mathfrak{M}^{P} \mid \omega(P)=1\right\} .
$$

For any normal state $\omega$ one clearly has $0<s_{\omega} \leq z_{\omega} \leq I$ and hence $\omega(A)=$ $\omega\left(A z_{\omega}\right)=\omega\left(z_{\omega} A\right)$ for all $A \in \mathfrak{M}$. The state $\omega$ is centrally faithful if $z_{\omega}=I$. Lemma 3 shows that the central support of the vector state $\omega_{\Phi}$ is

$$
z_{\omega_{\Phi}}=\left[\mathfrak{M} \vee \mathfrak{M}^{\prime} \Phi\right] .
$$

In particular, if $\Phi$ is cyclic for $\mathfrak{M}$ or $\mathfrak{M}^{\prime}$ or if $\mathfrak{M}$ is a factor, then $\omega_{\Phi}$ is centrally faithful. More generally one has 
Lemma 5. Let $\omega$ be a normal state on the von Neumann algebra $\mathfrak{M} \subset \mathfrak{B}(\mathcal{H})$. If $s_{\omega}$ is the support of $\omega$, its central support is given by

$$
z_{\omega}=\left[\mathfrak{M} s_{\omega} \mathcal{H}\right]
$$

Proof. Set $\mathcal{K} \equiv \overline{\mathfrak{M} s_{\omega} \mathcal{H}}$ and denote by $P$ the orthogonal projection on $\mathcal{K}$. We first claim that $P \in \mathfrak{Z}(\mathfrak{M})$. Using Proposition 10 in [7] and the relation $\mathfrak{Z}(\mathfrak{M})^{\prime}=\left(\mathfrak{M} \cup \mathfrak{M}^{\prime}\right)^{\prime \prime}$, this follows from the fact that $\mathfrak{M} s_{\omega} \mathcal{H}$ and hence $\mathcal{K}$ are invariant under $\mathfrak{M}$ and $\mathfrak{M}^{\prime}$. Next we note that $\operatorname{Ran} s_{\omega} \subset \mathcal{K}$ implies $P \geq s_{\omega}$ and hence $\omega(P)=1$. Finally, if $Q \in \mathfrak{M}^{P} \cap \mathfrak{Z}(\mathfrak{M})$ is such that $\omega(Q)=1$, CauchySchwarz inequality yields that $\omega(A(I-Q))=0$ for all $A \in \mathfrak{M}$. Exercise 7 further leads to $(I-Q) A s_{\omega}=A(I-Q) s_{\omega}=0$ for all $A \in \mathfrak{M}$. This shows that $(I-Q) P=0$, i.e., $Q \geq P$ and Equ. (5) yields that $P=z_{\omega}$.

By Corollary 1 , a $*$-automorphism $\tau$ of a von Neumann algebra is automatically continuous in the $\sigma$-weak topology. In particular $\omega \circ \tau$ is a normal state for any normal state $\omega$. It immediately follows from the definitions that $s_{\omega \circ \tau}=\tau^{-1}\left(s_{\omega}\right)$ and $z_{\omega \circ \tau}=\tau^{-1}\left(z_{\omega}\right)$.

\subsection{The GNS Representation}

Let $\mathfrak{A}$ be a $C^{*}$-algebra and $\omega \in E(\mathfrak{A})$. Throughout these notes I shall use the standard notation $\left(\mathcal{H}_{\omega}, \pi_{\omega}, \Omega_{\omega}\right)$ for the GNS representation of $\mathfrak{A}$ associated to the state $\omega$ (Theorem 8 in Lecture [7]).

\section{Enveloping von Neumann Algebra and Folium of a State}

Since $\Omega_{\omega}$ is cyclic for $\pi_{\omega}(\mathfrak{A})$,

$$
\hat{\omega}(A) \equiv\left(\Omega_{\omega}, A \Omega_{\omega}\right),
$$

defines a centrally faithful normal state on the von Neumann algebra $\pi_{\omega}(\mathfrak{A})^{\prime \prime}$.

By the von Neumann density theorem, $\pi_{\omega}(\mathfrak{A})$ is $\sigma$-weakly dense in $\pi_{\omega}(\mathfrak{A})^{\prime \prime}$ and hence we have a canonical injection

$$
\begin{aligned}
N\left(\pi_{\omega}(\mathfrak{A})^{\prime \prime}\right) & \rightarrow E(\mathfrak{A}) \\
\tilde{\nu} \hookrightarrow & \pi_{\omega}^{\star}(\tilde{\nu})=\tilde{\nu} \circ \pi_{\omega} .
\end{aligned}
$$

Thus, we can identify $N\left(\pi_{\omega}(\mathfrak{A})^{\prime \prime}\right)$ with a subset $N(\mathfrak{A}, \omega)$ of $E(\mathfrak{A})$. Explicitly, $\nu \in N(\mathfrak{A}, \omega)$ if and only if there exists a density matrix $\rho$ on $\mathcal{H}_{\omega}$ and a corresponding normal state $\tilde{\nu}$ on $\pi_{\omega}(\mathfrak{A})^{\prime \prime}$ such that

$$
\nu(A)=\tilde{\nu} \circ \pi_{\omega}(A)=\operatorname{Tr}\left(\rho \pi_{\omega}(A)\right) .
$$

Definition 2. Let $\mathfrak{A}$ be a $C^{*}$-algebra and $\omega \in E(\mathfrak{A})$.

(i) $\mathfrak{A}_{\omega} \equiv \pi_{\omega}(\mathfrak{A})^{\prime \prime} \subset \mathfrak{B}\left(\mathcal{H}_{\omega}\right)$ is the enveloping von Neumann algebra of $\mathfrak{A}$ associated to $\omega$. 
(ii) $N(\mathfrak{A}, \omega) \subset E(\mathfrak{A})$ is the folium of the state $\omega$. It is the image under $\pi_{\omega}^{\star}$ of the set of states on $\pi_{\omega}(\mathfrak{A})$ which have a unique normal extension to the enveloping algebra $\mathfrak{A}_{\omega}$. Its elements are said to be normal relative to $\omega$, or simply $\omega$-normal.

Note that $\hat{\omega}$ is the unique normal extension of the state $\pi_{\omega}(A) \mapsto \omega(A)$ from $\pi_{\omega}(\mathfrak{A})$ to its weak closure $\mathfrak{A}_{\omega}$. By a slight abuse of language I shall say that $\hat{\omega}$ is the normal extension of $\omega$ to $\mathfrak{A}_{\omega}$. Similarly, if $\nu=\tilde{\nu} \circ \pi_{\omega} \in N(\mathfrak{A}, \omega)$ I shall say that $\tilde{\nu}$ is the normal extension of $\nu$ to $\mathfrak{A}_{\omega}$. I further denote by $s_{\nu \mid \omega}$ the support of $\tilde{\nu}$ and by $z_{\nu \mid \omega}$ its central support. Abusing notation, I also set $s_{\omega} \equiv s_{\omega \mid \omega}=s_{\hat{\omega}}$.

Definition 3. Let $\omega, \nu$ be two states on the $C^{*}$-algebra $\mathfrak{A}$.

(i) $\nu, \omega$ are quasi-equivalent, written $\nu \approx \omega$, if $N(\mathfrak{A}, \nu)=N(\mathfrak{A}, \omega)$.

(ii) They are orthogonal, written $\nu \perp \omega$, if $\lambda \mu \leq \nu$ and $\lambda \mu \leq \omega$ for some $\mu \in E(\mathfrak{A})$ and $\lambda \geq 0$ implies $\lambda=0$.

(iii) They are disjoint if $N(\mathfrak{A}, \nu) \cap N(\mathfrak{A}, \omega)=\varnothing$.

\section{The GNS Representation of a Normal State}

In this subsection we study the special features of the GNS representation associated to a normal state $\omega$ on the von Neumann algebra $\mathfrak{M} \subset \mathfrak{B}(\mathcal{H})$.

The first result relates the central support $z_{\omega}$ of the state $\omega$ to the kernel of the $*$-morphism $\pi_{\omega}$. Before stating this relation let me make the following remark.

Remark 2. If $P \in \mathfrak{Z}(\mathfrak{M})$ is an orthogonal projection then $Q \equiv I-P \in \mathfrak{Z}(\mathfrak{M})$ and since $\mathfrak{M P H}=P \mathfrak{M H} \subset P \mathcal{H}$ and $\mathfrak{M} Q \mathcal{H}=Q \mathfrak{M H} \subset Q \mathcal{H}$, any element of $\mathfrak{M}$ can be written, according to the orthogonal decomposition $\mathcal{H}=P \mathcal{H} \oplus Q \mathcal{H}$, as a $2 \times 2$-matrix

$$
A=\left(\begin{array}{cc}
B & 0 \\
0 & C
\end{array}\right)
$$

where $B \in \mathfrak{B}(P \mathcal{H})$ and $C \in \mathfrak{B}(Q \mathcal{H})$. Using the injection

$$
P \mathfrak{M} \ni\left(\begin{array}{cc}
B & 0 \\
0 & 0
\end{array}\right) \hookrightarrow B \in \mathfrak{B}(P \mathcal{H})
$$

we can identify $P \mathfrak{M}$ with a von Neumann algebra on $P \mathcal{H}$ and similarly for $Q \mathfrak{M}$. We then write $\mathfrak{M}=P \mathfrak{M} \oplus Q \mathfrak{M}$, and say that $\mathfrak{M}$ is the direct sum of the von Neumann algebras $P \mathfrak{M}$ and $Q \mathfrak{M}$. Of course the same argument applies to the commutant and we also have $\mathfrak{M}^{\prime}=P \mathfrak{M}^{\prime} \oplus Q \mathfrak{M}^{\prime}$. It follows immediately that $P \mathfrak{M}^{\prime}=(P \mathfrak{M})^{\prime}$ and $Q \mathfrak{M}^{\prime}=(Q \mathfrak{M})^{\prime}$ as von Neumann algebras on $P \mathcal{H}$ and $Q \mathcal{H}$. With the same interpretation we can write $\mathfrak{Z}(\mathfrak{M})=P \mathfrak{Z}(\mathfrak{M}) \oplus Q \mathfrak{Z}(\mathfrak{M})$ and $\mathfrak{Z}(P \mathfrak{M})=P \mathfrak{Z}(\mathfrak{M}), \mathfrak{Z}(Q \mathfrak{M})=Q \mathfrak{Z}(\mathfrak{M})$. 
Lemma 6. If $\omega \in N(\mathfrak{M})$ then $\operatorname{Ker}\left(\pi_{\omega}\right)=\left(I-z_{\omega}\right) \mathfrak{M}$. In particular, $\pi_{\omega}$ is faithful (i.e., is a *-isomorphism from $\mathfrak{M}$ onto $\pi_{\omega}(\mathfrak{M})$ ) if and only if $\omega$ is centrally faithful. More generally, the map

$$
\hat{\pi}_{\omega}: A z_{\omega} \mapsto \pi_{\omega}(A),
$$

defines a $*$-isomorphism from the von Neumann algebra $z_{\omega} \mathfrak{M}$ onto $\pi_{\omega}(\mathfrak{M})$ such that, for all $A \in \mathfrak{M}$ and all $B \in \pi_{\omega}(\mathfrak{M})$ one has $\pi_{\omega} \circ \hat{\pi}_{\omega}^{-1}(B)=B$ and $\hat{\pi}_{\omega}^{-1} \circ \pi_{\omega}(A)=z_{\omega} A$.

Proof. For $A, B \in \mathfrak{M}$ one has

$$
\left\|\pi_{\omega}\left(A\left(I-z_{\omega}\right)\right) \pi_{\omega}(B) \Omega_{\omega}\right\|^{2}=\omega\left(B^{*} A^{*} A B\left(I-z_{\omega}\right)\right)=0 .
$$

Since $\pi_{\omega}(\mathfrak{M}) \Omega_{\omega}$ is dense in $\mathcal{H}_{\omega}$ one concludes that $\pi_{\omega}\left(A\left(I-z_{\omega}\right)\right)=0$, i.e., $\mathfrak{M}\left(I-z_{\omega}\right) \subset \operatorname{Ker}\left(\pi_{\omega}\right)$.

To prove the reverse inclusion note that $\pi_{\omega}(A)=0$ implies that

$$
\omega\left(B^{*} A^{*} A B\right)=\left\|\pi_{\omega}(A) \pi_{\omega}(B) \Omega_{\omega}\right\|^{2}=0,
$$

for all $B \in \mathfrak{M}$. Exercise 7 further gives $A B s_{\omega}=0$ for all $B \in \mathfrak{M}$ and Lemma 5 yields $A z_{\omega}=0$, i.e., $A=A\left(I-z_{\omega}\right)$. The proof of the last statement of the lemma is easy and left to the reader

Corollary 2. Let $\mathfrak{M}$ be a von Neumann algebra and $\omega, \nu \in N(\mathfrak{M})$. Then $\nu$ is $\omega$-normal if and only if $s_{\nu} \leq z_{\omega}$.

Proof. Suppose that $\nu=\hat{\nu} \circ \pi_{\omega}$ for some $\hat{\nu} \in N\left(\mathfrak{M}_{\omega}\right)$. By Lemma 6 we have

$$
\nu\left(I-z_{\omega}\right)=\hat{\nu}\left(\pi_{\omega}\left(I-z_{\omega}\right)\right)=\hat{\nu}(0)=0 .
$$

Thus, $\nu\left(z_{\omega}\right)=1$ from which we conclude that $s_{\nu} \leq z_{\omega}$.

Suppose now that $s_{\nu} \leq z_{\omega}$ and set $\hat{\nu} \equiv \nu \circ \hat{\pi}_{\omega}^{-1}$. Since $\hat{\pi}_{\omega}$ is a $*$-isomorphism $\hat{\nu}$ is normal. Moreover, from $\nu(A)=\nu\left(z_{\omega} A\right)$ we conclude that

$$
\hat{\nu} \circ \pi_{\omega}(A)=\nu\left(\hat{\pi}_{\omega}^{-1}\left(\pi_{\omega}(A)\right)\right)=\nu\left(z_{\omega} A\right)=\nu(A) .
$$

The continuity properties of $\pi_{\omega}$ follow from the simple lemma:

Lemma 7. The map $\pi_{\omega}$ is normal i.e., for any bounded increasing net $A_{\alpha}$ of self-adjoint elements of $\mathfrak{M}$ one has

$$
\sup _{\alpha} \pi_{\omega}\left(A_{\alpha}\right)=\pi_{\omega}\left(\sup _{\alpha} A_{\alpha}\right) .
$$


Proof. For any $B \in \mathfrak{M}$ one has $\omega\left(B^{*} A_{\alpha} B\right)=\left(\pi_{\omega}(B) \Omega_{\omega}, \pi_{\omega}\left(A_{\alpha}\right) \pi_{\omega}(B) \Omega_{\omega}\right)$ and Equ. (1) allows us to write

$$
\begin{aligned}
\left(\pi_{\omega}(B) \Omega_{\omega}, \sup _{\alpha} \pi_{\omega}\left(A_{\alpha}\right) \pi_{\omega}(B) \Omega_{\omega}\right) & =\sup _{\alpha}\left(\pi_{\omega}(B) \Omega_{\omega}, \pi_{\omega}\left(A_{\alpha}\right) \pi_{\omega}(B) \Omega_{\omega}\right) \\
& =\sup _{\alpha} \omega\left(B^{*} A_{\alpha} B\right) \\
& =\omega\left(B^{*}\left(\sup _{\alpha} A_{\alpha}\right) B\right) \\
& =\left(\pi_{\omega}(B) \Omega_{\omega}, \pi_{\omega}\left(\sup _{\alpha} A_{\alpha}\right) \pi_{\omega}(B) \Omega_{\omega}\right) .
\end{aligned}
$$

Since $\pi_{\omega}(\mathfrak{M}) \Omega_{\omega}$ is dense in $\mathcal{H}_{\omega}$ the claim follows.

Exercise 9. Prove the following lemma using Lemma 7 and following the proof of Corollary 1.

Lemma 8. If $\omega \in N(\mathfrak{M})$ then $\pi_{\omega}$ is $\sigma$-weakly and $\sigma$-strongly continuous.

Lemma 9. If $\omega \in N(\mathfrak{M})$ then $\pi_{\omega}(\mathfrak{M})$ is a von Neumann algebra in $\mathfrak{B}\left(\mathcal{H}_{\omega}\right)$, i.e., $\mathfrak{M}_{\omega}=\pi_{\omega}(\mathfrak{M})$.

Proof. Since $\pi_{\omega}(\mathfrak{M})=\hat{\pi}_{\omega}\left(\mathfrak{M} z_{\omega}\right)$ we can assume, without loss of generality, that $\pi_{\omega}$ is faithful and hence isometric (Proposition 4 in Lecture [7]). Let $B \in \pi_{\omega}(\mathfrak{M})^{\prime \prime}$. By the Kaplansky density theorem there exists a net $A_{\alpha}$ in $\mathfrak{M}$ such that $\left\|\pi_{\omega}\left(A_{\alpha}\right)\right\| \leq\|B\|$ and $\pi_{\omega}\left(A_{\alpha}\right)$ converges $\sigma$-weakly to $B$. Since

$$
\left\|A_{\alpha}\right\|=\left\|\pi_{\omega}\left(A_{\alpha}\right)\right\| \leq\|B\|
$$

it follows from the Banach-Alaoglu theorem that there exists a subnet $A_{\beta}$ of the net $A_{\alpha}$ which converges $\sigma$-weakly to some $A \in \mathfrak{M}$. Since $\pi_{\omega}$ is $\sigma$-weakly continuous one has

$$
\pi_{\omega}(A)=\lim _{\beta} \pi_{\omega}\left(A_{\beta}\right)=\lim _{\alpha} \pi_{\omega}\left(A_{\alpha}\right)=B,
$$

and hence $B \in \pi_{\omega}(\mathfrak{M})$.

Lemma 10. If $\omega \in N(\mathfrak{M})$ then

$$
N(\mathfrak{M}, \omega)=\left\{\nu \in N(\mathfrak{M}) \mid s_{\nu} \leq z_{\omega}\right\} \subset N(\mathfrak{M}) .
$$

In particular, if $\omega$ is centrally faithful then $N(\mathfrak{M}, \omega)=N(\mathfrak{M})$.

Proof. By Lemma 8, if $\omega \in N(\mathfrak{M})$ then $\pi_{\omega}$ is $\sigma$-weakly continuous. Hence $N(\mathfrak{M}, \omega) \subset N(\mathfrak{M})$ and Corollary 2 apply.

As an application of the above results let us prove the following characterization of the relative normality of two states on a $C^{*}$-algebra. 
Theorem 4. Let $\mathfrak{A}$ be a $C^{*}$-algebra and $\omega, \mu \in E(\mathfrak{A})$. Denote the induced GNS representations by $\left(\mathcal{H}_{\omega}, \pi_{\omega}, \Omega_{\omega}\right),\left(\mathcal{H}_{\mu}, \pi_{\mu}, \Omega_{\mu}\right)$ and the corresponding enveloping von Neumann algebras by $\mathfrak{A}_{\omega}, \mathfrak{A}_{\mu}$. Then $\mu \in N(\mathfrak{A}, \omega)$ if and only if there exists a $\sigma$-weakly continuous $*$-morphism $\pi_{\mu \mid \omega}: \mathfrak{A}_{\omega} \rightarrow \mathfrak{A}_{\mu}$ such that $\pi_{\mu}=\pi_{\mu \mid \omega} \circ \pi_{\omega}$. If this is the case then the following also hold.

(i) $N(\mathfrak{A}, \mu) \subset N(\mathfrak{A}, \omega)$.

(ii) If $\nu \in N(\mathfrak{A}, \mu)$ has the normal extension $\tilde{\nu}$ to $\mathfrak{A}_{\mu}$ then $\tilde{\nu} \circ \pi_{\mu \mid \omega}$ is its normal extension to $\mathfrak{A}_{\omega}$.

(iii) $\pi_{\mu \mid \omega}$ is $\sigma$-strongly continuous.

(iv) $\hat{\pi}_{\mu \mid \omega}: z_{\mu \mid \omega} A \mapsto \pi_{\mu \mid \omega}(A)$ is a *-isomorphism from $z_{\mu \mid \omega} \mathfrak{A}_{\omega}$ onto $\mathfrak{A}_{\mu}$.

(v) $\operatorname{Ker} \pi_{\mu \mid \omega}=\left(I-z_{\mu \mid \omega}\right) \mathfrak{A}_{\omega}$

(vi) $\pi_{\mu \mid \omega}\left(s_{\mu \mid \omega}\right)=s_{\mu}$.

(vii) If $\tilde{\mu}$ denotes the normal extension of $\mu$ to $\mathfrak{A}_{\omega}$ then $\left(\mathcal{H}_{\mu}, \pi_{\mu \mid \omega}, \Omega_{\mu}\right)$ is the induced GNS representation of $\mathfrak{A}_{\omega}$. In particular $\mathfrak{A}_{\mu}=\left(\mathfrak{A}_{\omega}\right)_{\hat{\mu}}$.

Proof. Let $\hat{\mu}(A) \equiv\left(\Omega_{\mu}, A \Omega_{\mu}\right)$ be the normal extension of $\mu$ to $\mathfrak{A}_{\mu}$. If the morphism $\pi_{\mu \mid \omega}$ exists then one has $\mu=\hat{\mu} \circ \pi_{\mu}=\left(\hat{\mu} \circ \pi_{\mu \mid \omega}\right) \circ \pi_{\omega}$. Since $\pi_{\mu \mid \omega}$ is $\sigma$-weakly continuous $\hat{\mu} \circ \pi_{\mu \mid \omega} \in N\left(\mathfrak{A}_{\omega}\right)$ and we conclude that $\mu \in N(\mathfrak{A}, \omega)$.

Assume now that $\mu \in N(\mathfrak{A}, \omega)$, i.e., that $\mu=\tilde{\mu} \circ \pi_{\omega}$ with $\tilde{\mu} \in N\left(\mathfrak{A}_{\omega}\right)$. Consider the GNS representation $(\mathcal{K}, \phi, \Psi)$ of $\mathfrak{A}_{\omega}$ induced by $\tilde{\mu}$. By Lemma 8 $\phi$ is $\sigma$-weakly and $\sigma$-strongly continuous. Since by the von Neumann density theorem $\pi_{\omega}(\mathfrak{A})$ is $\sigma$-strongly dense in $\mathfrak{A}_{\omega}$, we get

$$
\mathcal{K}=\overline{\phi\left(\mathfrak{A}_{\omega}\right) \Psi}=\overline{\phi\left(\pi_{\omega}(\mathfrak{A})\right) \Psi} .
$$

Finally, for any $A \in \mathfrak{A}$ one has

$$
\mu(A)=\tilde{\mu}\left(\pi_{\omega}(A)\right)=\left(\Psi, \phi\left(\pi_{\omega}(A)\right) \Psi\right),
$$

and we can conclude that $\left(\mathcal{K}, \phi \circ \pi_{\omega}, \Psi\right)$ is a GNS representation of $\mathfrak{A}$ induced by $\mu$. By the unicity, up to unitary equivalence, of such representations there exists a unitary $U: \mathcal{K} \rightarrow \mathcal{H}_{\mu}$ such that $\pi_{\mu}(A)=U \phi\left(\pi_{\omega}(A)\right) U^{*}$ and $\Omega_{\mu}=U \Psi$. Set $\pi_{\mu \mid \omega}(A) \equiv U \phi(A) U^{*}$ then $\left(\mathcal{H}_{\mu}, \pi_{\mu \mid \omega}, \Omega_{\mu}\right)$ is another GNS representation of $\mathfrak{A}_{\omega}$ induced by $\tilde{\mu}$ and $\pi_{\mu}=\pi_{\mu \mid \omega} \circ \pi_{\omega}$. Since $\pi_{\mu \mid \omega}$ is also $\sigma$-weakly and $\sigma$-strongly continuous Lemma 9 and the von Neumann density theorem yield

$$
\left(\mathfrak{A}_{\omega}\right)_{\tilde{\mu}}=\pi_{\mu \mid \omega}\left(\mathfrak{A}_{\omega}\right)=\pi_{\mu \mid \omega}\left(\pi_{\omega}(\mathfrak{A})\right)^{\prime \prime}=\pi_{\mu}(\mathfrak{A})^{\prime \prime}=\mathfrak{A}_{\mu} .
$$

This proves the existence of $\pi_{\mu \mid \omega}$ with Properties (iii) and (vii).

To prove Properties (i) and (ii) let $\nu \in N(\mathfrak{A}, \mu)$ and denote by $\tilde{\nu}$ its normal extension to $\mathfrak{A}_{\mu}$. One has $\nu=\tilde{\nu} \circ \pi_{\mu}=\tilde{\nu} \circ \pi_{\mu \mid \omega} \circ \pi_{\omega}$ and it follows that $\nu \in N(\mathfrak{A}, \omega)$ and that its normal extension to $\mathfrak{A}_{\omega}$ is $\tilde{\nu} \circ \pi_{\mu \mid \omega}$. Properties (iv) and (v) follow directly from Lemma 6 . By Lemma $7, \pi_{\mu \mid \omega}$ is normal and hence

$$
\begin{aligned}
\pi_{\mu \mid \omega}\left(s_{\mu \mid \omega}\right) & =\pi_{\mu \mid \omega}\left(\inf \left\{P \in \mathfrak{A}_{\omega}^{P} \mid \tilde{\mu}(P)=1\right\}\right) \\
& =\inf \left\{\pi_{\mu \mid \omega}(P) \mid P \in \mathfrak{A}_{\omega}^{P}, \hat{\mu}\left(\pi_{\mu \mid \omega}(P)\right)=1\right\} \\
& =\inf \left\{Q \in \mathfrak{A}_{\mu}^{P} \mid \hat{\mu}(Q)=1\right\} \\
& =s_{\mu},
\end{aligned}
$$


proves Property (vi).

\section{Classical Systems}

\subsection{Basics of Ergodic Theory}

Let $X$ be a measurable space, i.e., a set equipped with a $\sigma$-field $\mathcal{F}$. A dynamics on $X$ is a family of maps $\varphi_{t}: X \rightarrow X$, indexed by a time $t$ running in $\mathbb{R}$, such that $(x, t) \mapsto \varphi_{t}(x)$ is measurable and the group properties

$$
\varphi_{0}(x)=x, \quad \varphi_{t} \circ \varphi_{s}=\varphi_{t+s}
$$

hold. In particular, the map $\varphi_{t}$ is one to one with inverse $\varphi_{-t}$. Given $x \in X$, we set $x_{t}=\varphi_{t}(x)$ and call $\left(x_{t}\right)_{t \in \mathbb{R}} \subset X$ the orbit or trajectory starting at $x$. Observables are bounded measurable functions $f: X \rightarrow \mathbb{C}$.

Instead of considering individual orbits, think of the initial configuration $x$ as a random variable distributed according to a probability measure ${ }^{1} \mu$ on $X$. Then $\left(x_{t}\right)_{t \in \mathbb{R}}$ becomes a stochastic process and we denote by $\mathbb{E}_{\mu}$ the corresponding mathematical expectation. If $f$ is an observable, $\mathbb{E}_{\mu}\left[f\left(x_{0}\right)\right]=$ $\mu(f)=\int f \mathrm{~d} \mu$ is the expectation of $f$ at time zero. Its expectation at time $t$ is $\mu_{t}(f) \equiv \mathbb{E}_{\mu}\left[f\left(x_{t}\right)\right]=\mu\left(f \circ \varphi_{t}\right)$ which defines the evolution $\mu_{t}$ of the measure $\mu$. A measure $\mu$ is called invariant if the corresponding process is stationary, i.e., if $\mu_{t}=\mu$ for all $t$. Such an invariant measure describes a stationary regime of the system, or an equilibrium state.

Invariant probabilities may fail to exist (see Exercise I.8.6 in [35]) but most physical systems of interest have a lot of them. For example, if $x \in X$ is periodic of period $T$, then

$$
\mu_{x} \equiv \frac{1}{T} \int_{0}^{T} \delta_{x_{t}} \mathrm{~d} t
$$

is invariant, supported by the orbit of $x$. Under additional topological assumptions, one can prove that there is at least one invariant measure.

Exercise 10. Let $M$ be a compact metric space and $\varphi_{t}$ a continuous dynamics on $M$, i.e., assume that the map $(t, x) \mapsto \varphi_{t}(x)$ is continuous. Recall the following facts: The set of continuous observables $C(M)$ is a separable Banach space. Its dual $C(M)^{\star}$ is the set of Baire measures on $M$. The set of

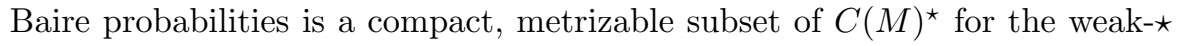
topology. Every Baire measure has a unique regular Borel extension.

Show that for any Baire probability $\mu$ on $M$, there exists a sequence $T_{n} \rightarrow$ $+\infty$, such that the weak- $\star$ limit

\footnotetext{
${ }^{1}$ All measures in this section are probabilities, so I use the words measure and probability interchangeably.
} 


$$
\mu^{+}=\mathrm{w}^{\star}-\lim \frac{1}{T_{n}} \int_{0}^{T_{n}} \mu_{t} \mathrm{~d} t
$$

exists and defines an invariant probability for $\varphi_{t}$ on $M$.

Definition 4. A classical dynamical system is a triple $\left(X, \varphi_{t}, \mu\right)$ where $X$, the phase space of the system, is a measurable space, $\varphi_{t}$ a dynamics on $X$ and $\mu$ an invariant measure for $\varphi_{t}$.

If $\left(X, \varphi_{t}, \mu\right)$ is a classical dynamical system, then $U^{t} f \equiv f \circ \varphi_{t}$ defines a group of isometries on each Banach space $L^{p}(X, \mathrm{~d} \mu)$. Indeed, for any $f \in$ $L^{p}(X, \mathrm{~d} \mu)$ we have $U^{0} f=f \circ \varphi_{0}=f$,

$$
U^{t} U^{s} f=U^{t}\left(f \circ \varphi_{s}\right)=\left(f \circ \varphi_{s}\right) \circ \varphi_{t}=f \circ\left(\varphi_{s} \circ \varphi_{t}\right)=f \circ \varphi_{t+s}=U^{t+s} f,
$$

and

$$
\left\|U^{t} f\right\|_{p}^{p}=\mu\left(\left|f \circ \varphi_{t}\right|^{p}\right)=\mu\left(|f|^{p} \circ \varphi_{t}\right)=\mu_{t}\left(|f|^{p}\right)=\mu\left(|f|^{p}\right)=\|f\|^{p} .
$$

A function $f \in L^{p}(X, \mathrm{~d} \mu)$ is invariant if $U^{t} f=f$ for all $t$. A measurable set $A \subset X$ is invariant modulo $\mu$ if its characteristic function $\chi_{A}$ is invariant, that is

$$
0=\left|\chi_{A}-U^{t} \chi_{A}\right|=\left|\chi_{A}-\chi_{\varphi_{t}^{-1}(A)}\right|=\chi_{A \Delta \varphi_{t}^{-1}(A)},
$$

which is equivalent to $\mu\left(A \Delta \varphi_{t}^{-1}(A)\right)=0$.

Ergodic theory deals with the study of invariant measures and their connections with the large time behavior of dynamical system. The cornerstone of ergodic theory is the following, so called Birkhoff or individual ergodic theorem.

Theorem 5. Let $\left(X, \varphi_{t}, \mu\right)$ be a classical dynamical system. Then for any $f \in L^{1}(X, \mathrm{~d} \mu)$, the two limits

$$
\left(\mathbb{P}_{\mu} f\right)(x)=\lim _{T \rightarrow \infty} \frac{1}{T} \int_{0}^{T} f \circ \varphi_{ \pm t}(x) \mathrm{d} t,
$$

exist and coincide for $\mu$-almost all $x \in X$. They define a linear contraction $\mathbb{P}_{\mu}$ on $L^{1}(X, \mathrm{~d} \mu)$ with the following properties:

(i) $\mathbb{P}_{\mu} f \geq 0$ if $f \geq 0$,

(ii) $\mathbb{P}_{\mu}^{2}=\mathbb{P}_{\mu}$,

(iii) $U^{t} \mathbb{P}_{\mu}=\mathbb{P}_{\mu} U^{t}=\mathbb{P}_{\mu}$ for all $t$,

(iv) $\mu(f)=\mu\left(\mathbb{P}_{\mu} f\right)$,

(v) if $g \in L^{1}(X, \mathrm{~d} \mu)$ is invariant, then $\mathbb{P}_{\mu} g f=g \mathbb{P}_{\mu} f$. In particular, $\mathbb{P}_{\mu} g=g$.

Remark 3. $\mathbb{P}_{\mu}$ is a conditional expectation with respect to the $\sigma$-field of invariant sets modulo $\mu$. 
Even in the case of a smooth (continuous or analytic) dynamical system, the conditional expectation $\mathbb{P}_{\mu} f(x)$ can display a weird dependence on the starting point $x$, reflecting the complexity of the orbits. Of special interest are the measures $\mu$ for which $\mathbb{P}_{\mu} f(x)$ is $\mu$-almost surely independent of $x$.

Definition 5. The dynamical system $\left(X, \varphi_{t}, \mu\right)$ is called ergodic if, for all $f \in L^{1}(X, \mathrm{~d} \mu)$, one has $\mathbb{P}_{\mu} f(x)=\mu(f)$ for $\mu$-almost all $x \in X$. In this case, we also say that $\mu$ is an ergodic measure for $\varphi_{t}$, or that the dynamics $\varphi_{t}$ is ergodic for $\mu$.

Proposition 1. The following propositions are equivalent.

(i) $\left(X, \varphi_{t}, \mu\right)$ is ergodic.

(ii) For any measurable set $A$ invariant modulo $\mu$, one has $\mu(A) \in\{0,1\}$.

(iii) For any invariant $f \in L^{1}(X, \mathrm{~d} \mu)$ one has $f=\mu(f)$.

(iv) For any $\mu$-absolutely continuous probability $\rho$ one has

$$
\lim _{T \rightarrow \infty} \frac{1}{T} \int_{0}^{T} \rho_{t}(f) \mathrm{d} t=\mu(f),
$$

for all $f \in L^{\infty}(X, \mathrm{~d} \mu)$.

Proof. Let us first show that (i), (ii) and (iii) are equivalent.

(i) $\Rightarrow$ (ii). If $A$ is invariant modulo $\mu$ then its indicator function $\chi_{A}$ is invariant. Property $(\mathrm{v})$ of Theorem 5 shows that $\chi_{A}=\mathbb{P}_{\mu} \chi_{A}$. If $\mu$ is ergodic we further have $\mathbb{P}_{\mu} \chi_{A}=\mu(A)$. Therefore, $\chi_{A}=\mu(A)$ and we conclude that $\mu(A) \in\{0,1\}$.

(ii) $\Rightarrow$ (iii). If $f \in L^{1}(X, \mathrm{~d} \mu)$ is invariant so are its real and imaginary parts. Without loss of generality we can assume that $f$ is real valued. Then the set $\{x \mid f(x)>a\}$ is invariant modulo $\mu$. Therefore, the distribution function $F_{f}(a)=\mu(\{x \mid f(x)>a\})$ takes values in the set $\{0,1\}$ from which we conclude that $f$ is constant $\mu$-almost everywhere.

(iii) $\Rightarrow\left(\right.$ i). If $f \in L^{1}(X, \mathrm{~d} \mu)$ then $\mathbb{P}_{\mu} f$ is invariant by property (iii) of Theorem 5 . Therefore, $\mathbb{P}_{\mu} f=\mu\left(\mathbb{P}_{\mu} f\right)$ and by property (iv) of Theorem 5 this is equal to $\mu(f)$.

Now we consider property (iv). Denote by $g \in L^{1}(X, \mathrm{~d} \mu)$ the RadonNikodym derivative of $\rho$ with respect to $\mu$. Then, for any $f \in L^{\infty}(X, \mathrm{~d} \mu)$, Fubini Theorem and the invariance of $\mu$ give

$$
\begin{aligned}
\frac{1}{T} \int_{0}^{T} \rho_{t}(f) \mathrm{d} t & =\frac{1}{T} \int_{0}^{T}\left(\int g(x) f \circ \varphi_{t}(x) \mathrm{d} \mu(x)\right) \mathrm{d} t \\
& =\int\left(\frac{1}{T} \int_{0}^{T} g \circ \varphi_{-t}(x) \mathrm{d} t\right) f(x) \mathrm{d} \mu(x) .
\end{aligned}
$$

Birkhoff Theorem together with Lebesgue dominated convergence Theorem further lead to 


$$
\rho^{+}(f) \equiv \lim _{T \rightarrow \infty} \frac{1}{T} \int_{0}^{T} \rho_{t}(f) \mathrm{d} t=\mu\left(\left(\mathbb{P}_{\mu} g\right) f\right) .
$$

Therefore, if $\mu$ is ergodic, we obtain $\rho^{+}(f)=\mu(\mu(g) f)=\mu(f)$. Reciprocally, if $\rho^{+}=\mu$ for all $\mu$-absolutely continuous probabilities $\rho$, we can conclude that for all $g \in L^{1}(X, \mathrm{~d} \mu)$ such that $\mu(g)=1$ one has $\mathbb{P}_{\mu} g=1=\mu(g)$. This clearly implies that $\mu$ is ergodic.

The following ergodic decomposition theorem shows that ergodic measures are elementary building blocks of invariant measures.

Theorem 6. Let $M$ be a compact metric space and $\varphi_{t}$ a continuous dynamics on $M$. Then the set $E_{\varphi}(M)$ of invariant probabilities for $\varphi_{t}$ is a non-empty,

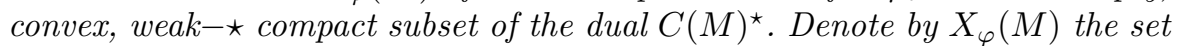
of extremal points ${ }^{2}$ of $E_{\varphi}(M)$ :

(i) $\mu \in X_{\varphi}(M)$ if and only if it is ergodic for $\varphi_{t}$.

(ii) If $\mu, \nu \in X_{\varphi}(M)$ and $\mu \neq \nu$, then $\mu$ and $\nu$ are mutually singular.

(iii) For any $\mu \in E_{\varphi}(M)$ there exists a probability measure $\rho$ on $X_{\varphi}(M)$ such that

$$
\mu=\int \nu \mathrm{d} \rho(\nu)
$$

The relevance of an ergodic measure $\mu$ in the study of the large time behavior of the system comes from the fact that the time average of an observable along a generic orbit is given by the ensemble average described by $\mu$ :

$$
\lim _{T \rightarrow \infty} \frac{1}{T} \int_{0}^{T} f\left(x_{t}\right) \mathrm{d} t=\mu(f),
$$

for $\mu$-almost all $x$. Note however that, depending on the nature of $\mu$, generic orbits may fill a very small portion of the phase space (as shown by the above example of a periodic orbit). Here small refers to some additional feature that the physical problem may induce on the space $X$. For example if $X$ is a smooth manifold, small could mean of measure zero with respect to some (any) Riemannian volume on $X$ (see [39] for a more detailed discussion of this important point).

A more precise information on the large time asymptotics of the system is given by the following mixing condition.

Definition 6. A dynamical system $\left(X, \varphi_{t}, \mu\right)$ is mixing if, for any $\mu$-absolutely continuous measure $\rho$ and all observables $f \in L^{\infty}(X, \mathrm{~d} \mu)$, one has

$$
\lim _{t \rightarrow \infty} \rho_{t}(f)=\mu(f) .
$$

One also says that $\mu$ is mixing, or $\varphi_{t}$ is mixing for $\mu$.

\footnotetext{
${ }^{2} \mu$ is extremal if it can not be expressed as a non-trivial convex linear combination of two distinct measures: $\mu=\alpha \mu_{1}+(1-\alpha) \mu_{2}$ with $\left.\alpha \in\right] 0,1\left[\right.$ and $\mu_{i} \in E_{\varphi}(M)$ implies $\mu_{1}=\mu_{2}=\mu$.
} 
If we think of an invariant measure $\mu$ as describing an equilibrium state of the system then $\mu$ is mixing if all initial measures $\rho$ which are not too far from equilibrium, i.e., which are absolutely continuous with respect to $\mu$, converge to $\mu$ as $t \rightarrow \infty$. For this reason, the mixing condition is often referred to as return to equilibrium.

If $\mu$ is mixing, it is obviously ergodic from Property (iv) in Theorem 1. Here is another proof. If $A$ is an invariant set modulo $\mu$ such that $\mu(A)>0$, then $\rho(f)=\mu\left(f \chi_{A}\right) / \mu(A)$ defines a $\mu$-absolutely continuous invariant measure with $\rho(A)=1$. Therefore, if $\mu$ is mixing we have

$$
1=\rho(A)=\rho_{t}(A)=\lim _{t \rightarrow \infty} \rho_{t}(A)=\mu(A) .
$$

From Theorem 1 we conclude that $\mu$ is ergodic. Thus mixing implies ergodicity, but the reverse is not true in general.

Exercise 11. Let $X=\mathbb{R} / \mathbb{Z}$ be the one dimensional torus, $\mu$ the measure induced on $X$ by the Lebesgue measure on $\mathbb{R}$ and $\varphi_{t}(x)=x+t(\bmod 1)$. Show that $\left(X, \varphi_{t}, \mu\right)$ is ergodic but not mixing.

Exercise 12. Show that $\left(X, \varphi_{t}, \mu\right)$ is mixing if and only if, for all measurable subsets $A, B \subset X$ one has

$$
\lim _{t \rightarrow \infty} \mu\left(\varphi_{t}^{-1}(A) \cap B\right)=\mu(A) \mu(B) .
$$

Ergodicity and mixing are only two elements of the so called ergodic hierarchy which contains many other properties like exponential mixing, $K$ system... The interested reader should consult the general references given in the Introduction.

\subsection{Classical Koopmanism}

Ergodicity and mixing can be quite difficult to prove in concrete situations. One of the more powerful tools to do it is the Koopman-von Neumann spectral theory that I shall now introduce. The Koopman space of the dynamical system $\left(X, \varphi_{t}, \mu\right)$ is the Hilbert space $\mathcal{H}=L^{2}(X, \mathrm{~d} \mu)$ on which the Koopman operators $U^{t}$ are defined by

$$
U^{t} f \equiv f \circ \varphi_{t}
$$

In the following, I shall always assume that the Koopman space is separable. This is the case for example if $X$ is a (locally) compact metric space equipped with its natural Borel structure.

Lemma 11. (Koopman Lemma) If $\mathcal{H}$ is separable, $U^{t}$ is a strongly continuous group of unitary operators on $\mathcal{H}$. 
Proof. We have already shown that $U^{t}$ is a group of isometries on $\mathcal{H}$. Since $U^{t} U^{-t}=I$ we have $\operatorname{Ran} U^{t}=\mathcal{H}$ and therefore $U^{t}$ is unitary. Finally, since $t \mapsto\left(f, U^{t} g\right)$ is measurable and $\mathcal{H}$ is separable, it follows from a well known result of von Neumann (Theorem VIII.9 in [41]) that the map $t \mapsto U^{t}$ is strongly continuous.

Remark 4. The separability condition is satisfied if $X$ is a finite dimensional manifold. In infinite dimensional cases it can often be replaced by a weak continuity assumption. Indeed, if the map $t \mapsto \int f(x) f\left(\varphi_{t}(x)\right) \mathrm{d} \mu(x)$ is continuous for all $f \in L^{2}(X, \mathrm{~d} \mu)$, then $U^{t}$ is strongly continuous since

$$
\left\|U^{t} f-f\right\|^{2}=2\left(\|f\|^{2}-R e\left(f, U^{t} f\right)\right) .
$$

By the Stone Theorem, there exists a self-adjoint operator $L$ on $\mathcal{H}$ such that

$$
U^{t}=\mathrm{e}^{-i t L} .
$$

We call $L$ the Liouvillean of the system. Note that $1 \in \mathcal{H}$ and $U^{t} 1=1$ for all $t$, from which we conclude that $1 \in \mathrm{D}(L)$ and $L 1=0$. In other words, 0 is always an eigenvalue of the Liouvillean, with the associated eigenfunction 1. The connection between Ker $L$ and ergodic theory is the content of the following von Neumann or mean ergodic theorem.

Theorem 7. Let $U^{t}=\mathrm{e}^{-i t A}$ be a strongly continuous group of unitaries on a Hilbert space $\mathcal{H}$ and denote by $P$ the orthogonal projection on $\operatorname{Ker} A$. Then, for any $f \in \mathcal{H}$,

$$
\lim _{T \rightarrow \infty} \frac{1}{T} \int_{0}^{T} U^{t} f \mathrm{~d} t=P f
$$

holds in $\mathcal{H}$.

Proof. Since $U^{t}$ is strongly continuous,

$$
\langle f\rangle_{T}=\frac{1}{T} \int_{0}^{T} U^{t} f \mathrm{~d} t
$$

is well defined as a Riemann integral. We first remark that for $f \in \operatorname{Ran} A$, we have

$$
U^{t} f=U^{t} A g=i \partial_{t} U^{t} g
$$

for some $g \in \mathrm{D}(A)$. Hence an explicit integration gives

$$
\lim _{T \rightarrow \infty}\langle f\rangle_{T}=\lim _{T \rightarrow \infty} \frac{i}{T}\left(U^{T}-I\right) g=0 .
$$

Using the simple estimate $\left\|\langle u\rangle_{T}-\langle v\rangle_{T}\right\| \leq\|u-v\|$, this result immediately extends to all $f \in \overline{\operatorname{Ran} A}=\operatorname{Ker} A^{\perp}$. Since for $f \in \operatorname{Ker} A$ we have $\langle f\rangle_{T}=f$, we get for arbitrary $f \in \mathcal{H}$

$$
\lim _{T \rightarrow \infty}\langle f\rangle_{T}=\lim _{T \rightarrow \infty}\langle P f\rangle_{T}+\lim _{T \rightarrow \infty}\langle(I-P) f\rangle_{T}=P f .
$$


Theorem 8. (Koopman Ergodicity Criterion) A dynamical system is ergodic if and only if 0 is a simple eigenvalue of its Liouvillean $L$.

Proof. Let $f \in \operatorname{Ker} L$, then $f$ is an invariant function in $L^{1}(X, \mathrm{~d} \mu)$ and, by Theorem 1, if $\mu$ is ergodic we must have $f=\mu(f) 1$. Thus, $\operatorname{Ker} L$ is one dimensional.

Assume now that $\operatorname{Ker} L$ is one dimensional. Let $A$ be an invariant set modulo $\mu$. It follows that $\chi_{A} \in \mathcal{H}$ is invariant and hence belongs to $\operatorname{Ker} L$. Thus, we have $\chi_{A}=\mu(A) 1$, from which we may conclude that $\mu(A) \in\{0,1\}$. Ergodicity of $\mu$ follows from Theorem 1 .

Theorem 9. (Koopman Mixing Criterion) A dynamical system is mixing if and only if

$$
\mathrm{w}-\lim _{t \rightarrow \infty} U^{t}=(1, \cdot) 1
$$

In particular, if the spectrum of the Liouvillean $L$ is purely absolutely continuous on $\{1\}^{\perp}$, then the system is mixing.

Proof. Assume first that the system is mixing, and set

$$
\mathcal{H}_{1+}=\{g \in \mathcal{H} \mid g \geq 0, \mu(g)=1\} .
$$

Since any $g \in \mathcal{H}_{1+}$ is the Radon-Nikodym derivative of some $\mu$-absolutely continuous probability $\rho$, we get for any $f \in L^{\infty}(X, \mathrm{~d} \mu)$

$$
\left(g, U^{t} f\right)=\rho_{t}(f) \rightarrow \mu(f)=(g, 1)(1, f),
$$

as $t \rightarrow \infty$. Since any $g \in \mathcal{H}$ is a finite linear combination of elements of $\mathcal{H}_{1+}$, Equ. (9) actually holds for all $g \in \mathcal{H}$ and $f \in L^{\infty}(X, \mathrm{~d} \mu)$. Finally, since both side of Equ. (9) are $\mathcal{H}$-continuous in $f$ uniformly in $t$, Equ. (8) follows from the fact that $L^{\infty}(M, \mathrm{~d} \mu)$ is dense in $\mathcal{H}$.

The reverse statement is proved in an analogous way. Suppose $\rho$ is a $\mu$-absolutely continuous probability and denote by $g$ its Radon-Nikodym derivative. Assuming for a while that $g \in \mathcal{H}$, we get from Equ. (8) that $\lim _{t \rightarrow \infty} \rho_{t}(f)=\mu(f)$ for all $f \in L^{\infty}(M, \mathrm{~d} \mu)$. Since $\rho_{t}(f)$ is $L^{1}$-continuous in $g$, uniformly in $t$, the desired result follows from the fact that $\mathcal{H}$ is dense in $L^{1}(M, \mathrm{~d} \mu)$.

To prove the last statement, we first remark that to obtain Equ. (8) it suffices to show that

$$
\lim _{t \rightarrow \infty}\left(f, U^{t} f\right)=0
$$

for all $f \in\{1\}^{\perp}$ (use the orthogonal decomposition $\mathcal{H}=\mathbb{C} \cdot 1 \oplus\{1\}^{\perp}$ and the polarization identity). If $L$ has purely absolutely continuous spectrum on $\{1\}^{\perp}$ then its spectral measure associated to $f \in\{1\}^{\perp}$ can be written as $d \nu_{f}(\lambda)=g(\lambda) \mathrm{d} \lambda$ for some function $g \in L^{1}(\mathbb{R})$. Therefore,

$$
\left(f, U^{t} f\right)=\int \mathrm{e}^{i t \lambda} g(\lambda) \mathrm{d} \lambda \rightarrow 0,
$$

as $t \rightarrow \infty$ by the Riemann-Lebesgue Lemma. 
Note that $\left(f, U^{t} f\right)$ is the Fourier transform of the spectral measure of $L$ associated to $f$. It is clear from the above proof that a dynamical system is mixing if and only if the Fourier transform of the spectral measure of its Liouvillean associated to any vector in $\{1\}^{\perp}$ vanishes at infinity. See Subsection 2.5 in Lecture [25].

Exercise 13. On the $n$-dimensional torus $\mathbb{T}^{n}=\mathbb{R}^{n} / \mathbb{Z}^{n}$, consider the dynam$\operatorname{ics} \varphi_{t}(x)=x+t \omega(\bmod 1)$ where $\omega=\left(\omega_{1}, \cdots, \omega_{n}\right) \in \mathbb{R}^{n}$. Show that the (Haar) measure $d \mu(x)=d x_{1} \cdots d x_{n}$ is invariant and compute the Liouvillean $L$ of $\left(\mathbb{T}^{n}, \varphi_{t}, \mu\right)$. Determine the spectrum of $L$ and discuss the ergodic properties of the system.

\section{Quantum Systems}

In the traditional description of quantum mechanics, a quantum system is completely determined by its Hilbert space $\mathcal{H}$ and its Hamiltonian $H$, a selfadjoint operator on $\mathcal{H}$. The Hilbert space $\mathcal{H}$ determines both, the set of observables and the set of states of the system. The Hamiltonian $H$ specifies its dynamics.

An observable is a bounded linear operator on $\mathcal{H}$. A states is specified by a "wave function", a unit vector $\Psi \in \mathcal{H}$, or more generally by a "density matrix", a non-negative, trace class operator $\rho$ on $\mathcal{H}$ with $\operatorname{Tr} \rho=1$. The state associated with the density matrix $\rho$ is the linear functional

$$
A \mapsto \rho(A) \equiv \operatorname{Tr}(\rho A),
$$

on the set of observables. As a state, a unit vector $\Psi$ is equivalent to the density matrix $\rho=(\Psi, \cdot) \Psi$. Such density matrices are characterized by $\rho^{2}=\rho$ and the corresponding states

$$
A \mapsto(\Psi, A \Psi),
$$

are called vector states.

The role played by quantum mechanical states is somewhat similar to the role played by probability distributions in classical dynamical systems. However, since there is no quantum mechanical phase space, there is nothing like a trajectory and no state corresponding to the Dirac measures $\delta_{x}$ of classical dynamical systems.

To any self-adjoint observable $A$, a state $\rho$ associate a probability measure on the spectrum of $A$

$$
\mathrm{d} \rho_{A}(a)=\rho\left(E_{A}(\mathrm{~d} a)\right),
$$

where $E_{A}(\cdot)$ is the projection valued spectral measure of $A$ given by the spectral theorem. The measure $\rho_{A}$ specifies the probability distribution for the outcome of a measure of the observable $A$. In particular, the expectation value of $A$ in the state $\rho$ is 


$$
\int a \mathrm{~d} \rho_{A}(a)=\rho(A)
$$

If two or more self-adjoint observables $A, B, \ldots$, commute, they have a joint spectral measure and a similar formula gives the joint probability distribution for the simultaneous measurement of $A, B, \ldots$ However, if $A$ and $B$ do not commute, it is not possible to measure them simultaneously and there is no joint probability distribution for them (see also Lecture [33] in this Volume and [13] or any textbook on quantum mechanics for more details).

By the spectral decomposition theorem for compact operators, a density matrix $\rho$ can be written as

$$
\rho=\sum_{j} p_{j}\left(\Psi_{j}, \cdot\right) \Psi_{j}
$$

where the $\Psi_{j}$ are eigenvectors of $\rho$ and form an orthonormal basis of $\mathcal{H}$ and the coefficients $p_{j}$ are the corresponding eigenvalues which satisfy

$$
0 \leq p_{j} \leq 1, \quad \sum_{j} p_{j}=1 .
$$

Thus, a general state is a convex linear combination of vector states. $p_{j}$ is the probability for the system to be in the vector state associated to $\Psi_{j}$. In the physics literature, such a state is sometimes called statistical mixture (or incoherent superposition) of the states $\Psi_{j}$ with the statistical weights $p_{j}$.

A wave function $\Psi$ evolves according to the Schrödinger equation of motion

$$
i \partial_{t} \Psi_{t}=H \Psi_{t} .
$$

Since $H$ is self-adjoint, the solution of this equation, with initial value $\Psi_{0}=\Psi$, is given by

$$
\Psi_{t}=\mathrm{e}^{-i t H} \Psi .
$$

According to the decomposition (11), a density matrix $\rho$ evolves as

$$
\rho_{t}=\mathrm{e}^{-i t H} \rho \mathrm{e}^{i t H},
$$

and thus, satisfies the quantum Liouville equation

$$
\partial_{t} \rho_{t}=i\left[\rho_{t}, H\right] .
$$

The expectation value of an observable $A$ at time $t$ is then

$$
\rho_{t}(A)=\operatorname{Tr}\left(\rho_{t} A\right) .
$$

This is the so-called Schrödinger picture of quantum dynamics. Since the cyclicity of the trace implies that

$$
\operatorname{Tr}\left(\rho_{t} A\right)=\operatorname{Tr}\left(\mathrm{e}^{-i t H} \rho \mathrm{e}^{i t H} A\right)=\operatorname{Tr}\left(\rho \mathrm{e}^{i t H} A \mathrm{e}^{-i t H}\right),
$$


we can alternatively keep the state fixed and let observables evolve according to

$$
A_{t}=\mathrm{e}^{i t H} A \mathrm{e}^{-i t H} .
$$

Such time evolved observables satisfy the Heisenberg equation of motion

$$
\partial_{t} A_{t}=i\left[H, A_{t}\right]
$$

We obtain in this way the Heisenberg picture of quantum dynamics. Since

$$
\rho_{t}(A)=\rho\left(A_{t}\right),
$$

the Schrödinger and the Heisenberg pictures are obviously equivalent.

For systems with a finite number of degrees of freedom, this Hilbert space description of a quantum system is good enough because it is essentially unique (a precise form of this statement is the Stone-von Neumann uniqueness theorem, see Lecture [36]). However, for systems with an infinite number of degrees of freedom, (i.e., quantum fields) this is no more the case. An intrinsic description, centered around the $C^{*}$-algebra of observables becomes more convenient.

\section{1 $C^{*}$-Dynamical Systems}

Definition 7. A $C^{*}$-dynamical system is a pair $\left(\mathfrak{A}, \tau^{t}\right)$ where $\mathfrak{A}$ is a $C^{*}$ algebra with a unit and $\tau^{t}$ a strongly continuous group of $*$-automorphisms of $\mathfrak{A}$.

Since $\tau^{t}\left((z-A)^{-1}\right)=\left(z-\tau^{t}(A)\right)^{-1}$, a $*$-automorphism $\tau^{t}$ clearly preserves the spectrum. Hence, given the relation between the norm and the spectral radius (see the proof of Theorem 3 in Lecture [7]), it is isometric and in particular norm continuous. Strong continuity of $\tau^{t}$ means that, for any $A \in \mathfrak{A}$, the map $t \mapsto \tau^{t}(A)$ is continuous in the norm topology of $\mathfrak{A}$.

From the general theory of strongly continuous semi-groups, there exists a densely defined, norm closed linear operator $\delta$ on $\mathfrak{A}$ such that $\tau^{t}=\mathrm{e}^{t \delta}$. Since $\tau^{t}(1)=1$, if follows that $1 \in \mathrm{D}(\delta)$ and $\delta(1)=0$. Differentiation of the identities $\tau^{t}(A B)=\tau^{t}(A) \tau^{t}(B)$ and $\tau^{t}\left(A^{*}\right)=\tau^{t}(A)^{*}$ for $A, B \in \mathrm{D}(\delta)$ further show that the generator $\delta$ is a $*$-derivation as defined by the following

Definition 8. Let $\mathfrak{A}$ be $a *$-algebra and $\mathfrak{D} \subset \mathfrak{A}$ a subspace. A linear operator $\delta: \mathfrak{D} \rightarrow \mathfrak{A}$ is called $*$-derivation if

(i) $\mathfrak{D}$ is a $*$-subalgebra of $\mathfrak{A}$.

(ii) $\delta(A B)=\delta(A) B+A \delta(B)$ for all $A, B \in \mathfrak{D}$.

(iii) $\delta\left(A^{*}\right)=\delta(A)^{*}$ for all $A \in \mathfrak{D}$.

Generators of strongly continuous groups of $*$-automorphisms are characterized by the following simple adaptation of the Hille-Yosida Theorem (see [11], Theorem 3.2.50). 
Proposition 2. Let $\mathfrak{A}$ be a $C^{*}$-algebra with a unit. A densely defined, closed operator $\delta$ on $\mathfrak{A}$ generates a strongly continuous group of $*$-automorphisms of $\mathfrak{A}$ if and only if:

(i) $\delta$ is a *-derivation.

(ii) $\operatorname{Ran}(\operatorname{Id}+\lambda \delta)=\mathfrak{A}$ for all $\lambda \in \mathbb{R} \backslash\{0\}$.

(iii) $\|A+\lambda \delta(A)\| \geqslant\|A\|$ for all $\lambda \in \mathbb{R}$ and $A \in \mathrm{D}(\delta)$.

If the $C^{*}$-algebra $\mathfrak{A}$ acts on a Hilbert space $\mathcal{H}$ then a dynamical group $\tau^{t}$ can be constructed from a group of unitary operators $U^{t}$ on $\mathcal{H}$

$$
\tau^{t}(A)=U^{t} A U^{t *} .
$$

Such $*$-automorphisms are called spatial. They are particularly pleasant since it is possible to lift most of their analysis to the Hilbert space $\mathcal{H}$ itself.

Example 1. (Finite quantum systems) Consider the quantum system with a finite number of degrees of freedom determined by the data $\mathcal{H}$ and $H$ as described at the beginning of this section. On the $C^{*}$-algebra $\mathfrak{A}=\mathfrak{B}(\mathcal{H})$ the dynamics is given by

$$
\tau^{t}(A)=\mathrm{e}^{i t H} A \mathrm{e}^{-i t H} .
$$

Exercise 14. Show that the group $\tau^{t}$ is strongly continuous if and only if $H$ is bounded.

Thus, if $\tau^{t}$ is strongly continuous it is automatically uniformly continuous and its generator is the bounded $*$-derivation

$$
\delta(A)=i[H, A],
$$

on $\mathfrak{B}(\mathcal{H})$. More specific examples are:

1. $N$-levels systems: $\mathcal{H}=\mathbb{C}^{N}$ and, without loss of generality, the Hamiltonian is a $N \times N$ diagonal matrix $H_{i j}=\epsilon_{i} \delta_{i j}$.

2. Lattice quantum systems: $\mathcal{H}=l^{2}\left(\mathbb{Z}^{d}\right)$ and the Hamiltonian is of tightbinding type

$$
(H \psi)(x)=\frac{1}{2 d} \sum_{|x-y|=1} \psi(y)+V(x) \psi(x),
$$

where $|x|$ denotes the Euclidean norm of $x=\left(x_{1}, \cdots, x_{d}\right) \in \mathbb{Z}^{d}$ and $V \in$ $l^{\infty}\left(\mathbb{Z}^{d}\right)$. See Example 4 in Subsection 5.2 of Lecture [6] for a continuation of this example.

Example 2. (The ideal Fermi gas) Let $\mathfrak{h}$ be a Hilbert space and $\Gamma_{-}(\mathfrak{h})$ the Fermionic Fock space over $\mathfrak{h}$. Recall that

$$
\Gamma_{-}(\mathfrak{h})=\bigoplus_{n \in \mathbb{N}} \Gamma_{-}^{(n)}(\mathfrak{h}),
$$


where $\Gamma_{-}^{(0)}(\mathfrak{h})=\mathbb{C}$ and $\Gamma_{-}^{(n)}(\mathfrak{h})=\mathfrak{h} \wedge \mathfrak{h} \wedge \cdots \wedge \mathfrak{h}$ is the $n$-fold totally antisymmetric tensor product of $\mathfrak{h}$. For $f \in \mathfrak{h}$, the action of the Fermionic creation operator $a^{*}(f)$ on a Slater determinant $f_{1} \wedge f_{2} \wedge \cdots \wedge f_{n} \in \Gamma_{-}^{(n)}(\mathfrak{h})$ is defined by

$$
a^{*}(f) f_{1} \wedge f_{2} \wedge \cdots \wedge f_{n}=\sqrt{n+1} f_{1} \wedge f_{2} \wedge \cdots \wedge f_{n} \wedge f,
$$

and is extended by linearity to the dense subspace

$$
\Gamma_{\text {fin }-}(\mathfrak{h})=\bigcup_{k \in \mathbb{N}} \bigoplus_{n=0}^{k} \Gamma_{-}^{(n)}(\mathfrak{h})
$$

of $\Gamma_{-}(\mathfrak{h})$. The annihilation operators are defined in a similar way starting from

$$
a(f) f_{1} \wedge f_{2} \wedge \cdots \wedge f_{n}=\sqrt{n} \sum_{k=1}^{n}\left(f, f_{k}\right) f_{1} \wedge \cdots f_{k-1} \wedge f_{k+1} \cdots \wedge f_{n} .
$$

A simple calculation shows that these operators satisfy $a^{*}(f) \psi=a(f)^{*} \psi$ as well as the Canonical Anti-commutation Relations (CAR)

$$
\begin{aligned}
\left\{a(f), a^{*}(g)\right\} \psi & =(f, g) \psi, \\
\left\{a^{*}(f), a^{*}(g)\right\} \psi & =0,
\end{aligned}
$$

for all $f, g \in \mathfrak{h}$ and $\psi \in \Gamma_{\text {fin }-}(\mathfrak{h})$. It immediately follows that

$$
\|a(f) \psi\|^{2}+\left\|a^{*}(f) \psi\right\|^{2}=\|f\|^{2}\|\psi\|^{2},
$$

from which we conclude that the closures of $a(f)$ and $a^{*}(f)$ are bounded operators on $\Gamma_{-}(\mathfrak{h})$. If we denote these extensions by the same symbols then $a^{*}(f)=a(f)^{*}$ and the canonical anti-commutation relations hold for all $\psi \in$ $\Gamma_{-}(\mathfrak{h})$.

Exercise 15. Prove that $\|a(f)\|=\left\|a^{*}(f)\right\|=\|f\|$ (Hint: Use the CAR to compute $\left(a^{*}(f) a(f)\right)^{2}$ and the $C^{*}$-property of the norm). Compute the spectrum of $a(f), a^{*}(f)$ and $a^{*}(f) a(f)$.

Let $\mathfrak{u}$ be a subspace of $\mathfrak{h}$, not necessarily closed, and denote by CAR $(\mathfrak{u})$ the $C^{*}$-algebra generated by the family $\{a(f) \mid f \in \mathfrak{u}\}$, i.e., the norm closure in $\mathfrak{B}\left(\Gamma_{-}(\mathfrak{h})\right)$ of the linear span of monomials $a^{*}\left(f_{1}\right) \cdots a^{*}\left(f_{n}\right) a\left(f_{n+1}\right) \cdots a\left(f_{m}\right)$ with $f_{j} \in \mathfrak{u}$. It follows from the CAR that this algebra has a unit. To any selfadjoint operator $h$ on $\mathfrak{h}$ we can associate the second quantization $U^{t}=\Gamma\left(\mathrm{e}^{i h t}\right)$ on the Fock space. By definition

$$
U^{t} f_{1} \wedge \cdots \wedge f_{n} \equiv \mathrm{e}^{i t h} f_{1} \wedge \cdots \wedge \mathrm{e}^{i t h} f_{n}
$$

and therefore $U^{t}$ is a strongly continuous unitary group on $\Gamma_{-}(\mathfrak{h})$. Denote by $\tau^{t}$ the corresponding group of spatial $*$ - automorphisms of $\mathfrak{B}\left(\Gamma_{-}(\mathfrak{h})\right)$. 
Exercise 16. Show that the generator $H=\mathrm{d} \Gamma(h)$ of $U^{t}$ is bounded if and only if $h$ is trace class.

Hence, by Exercise 14, if $h$ is not trace class then $\tau^{t}$ is not strongly continuous on $\mathfrak{B}\left(\Gamma_{-}(\mathfrak{h})\right)$. Let me show that its restriction to $\operatorname{CAR}(\mathfrak{u})$ is strongly continuous.

It follows from Equ. (12) that

$$
\tau^{t}\left(a^{*}(f)\right)=U^{t} a^{*}(f) U^{t *}=a^{*}\left(\mathrm{e}^{i t h} f\right) .
$$

Therefore, for any monomial $A=a^{\#}\left(f_{1}\right) \cdots a^{\#}\left(f_{m}\right)$, where $a^{\#}$ stands for either $a$ or $a^{*}$, we get a telescopic expansion

$$
\begin{aligned}
\tau^{t}(A) & -A \\
& =\sum_{k=1}^{m} a^{\#}\left(\mathrm{e}^{i t h} f_{1}\right) \cdots a^{\#}\left(\mathrm{e}^{i t h} f_{k-1}\right) a^{\#}\left(\mathrm{e}^{i t h} f_{k}-f_{k}\right) a^{\#}\left(f_{k+1}\right) \cdots a^{\#}\left(f_{m}\right),
\end{aligned}
$$

which, together with Exercise 15, leads to the estimate

$$
\left\|\tau^{t}(A)-A\right\| \leqslant m\left(\max _{1 \leqslant k \leqslant m}\left\|f_{k}\right\|\right)^{m-1} \max _{1 \leqslant k \leqslant m}\left\|\mathrm{e}^{i t h} f_{k}-f_{k}\right\| .
$$

We conclude that $\lim _{t \rightarrow 0}\left\|\tau^{t}(A)-A\right\|=0$ for all such monomials and hence for arbitrary polynomials. Since these polynomials are norm dense in $\operatorname{CAR}(\mathfrak{u})$ and $\tau^{t}$ is isometric, the result follows from an $\varepsilon / 3$-argument.

The simplest non-trivial example of strongly continuous group of *automorphisms of $\operatorname{CAR}(\mathfrak{u})$ is the gauge group $\vartheta^{t}$ obtained by setting $h=I$, i.e.,

$$
\vartheta^{t}\left(a^{\#}(f)\right)=a^{\#}\left(\mathrm{e}^{i t} f\right) .
$$

The corresponding operator $N \equiv \mathrm{d} \Gamma(I)$ is the number operator.

The one particle Hilbert space for an infinite $d$-dimensional Fermi gas is $\mathfrak{h}=L^{2}\left(\mathbb{R}^{d}, \mathrm{~d} x\right)$. The non-relativistic Hamiltonian is $h=-\Delta$. To any compact region $\Lambda \Subset \mathbb{R}^{d}$ one can associate the Hilbert space $\mathfrak{h}_{\Lambda}=L^{2}(\Lambda, \mathrm{d} x)$ which is canonically embedded in $\mathfrak{h}$. Accordingly, the corresponding $C^{*}$-algebra $\operatorname{CAR}\left(\mathfrak{h}_{\Lambda}\right)$ can be identified with a subalgebra of $\operatorname{CAR}(\mathfrak{h})$. Elements of

$$
\mathfrak{A}_{\mathrm{loc}}=\bigcup_{\Lambda \Subset \mathbb{R}^{d}} \operatorname{CAR}\left(\mathfrak{h}_{\Lambda}\right),
$$

are called local and we say that $A \in \mathrm{CAR}\left(\mathfrak{h}_{\Lambda}\right)$ is supported by $\Lambda$.

Exercise 17. Show that $\mathfrak{A}_{\text {loc }}$ is a dense $*$-subalgebra of CAR $(\mathfrak{h})$.

From a physical point of view, local observables supported by disjoint subsets should be simultaneously measurable, i.e., they should commute. However, it follows from the fact that 


$$
\left\{A B, C^{\prime}\right\}=A\left\{B, C^{\prime}\right\}-\left\{A, C^{\prime}\right\} B
$$

that $\operatorname{CAR}\left(\mathfrak{h}_{\Lambda}\right)$ and $\operatorname{CAR}\left(\mathfrak{h}_{\Lambda^{\prime}}\right)$ anti-commute if $\Lambda \cap \Lambda^{\prime}=\varnothing$ and will generally not commute. Thus the full CAR algebra is in a sense too big. To obtain an algebra fulfilling the above locality requirement, one introduces the so called even subalgebra $\mathrm{CAR}^{+}(\mathfrak{h})$ generated by monomials of even degrees in $a$ and $a^{*}$. It follows from

$$
\left[A B, C^{\prime} D^{\prime}\right]=\left\{A, C^{\prime}\right\} D^{\prime} B+A\left\{B, C^{\prime}\right\} D^{\prime}-C^{\prime}\left\{D^{\prime}, A\right\} B-A C^{\prime}\left\{B, D^{\prime}\right\},
$$

that two elements of this subalgebra supported by disjoint subset commute.

Another way to characterize the even subalgebra is to consider the *morphism of $\operatorname{CAR}(\mathfrak{h})$ defined by $\theta(a(f))=-a(f)$. Then one clearly has

$$
\mathrm{CAR}^{+}(\mathfrak{h})=\{A \in \operatorname{CAR}(\mathfrak{h}) \mid \theta(A)=A\} .
$$

Since $\tau^{t}$ commutes with $\theta$, it leaves $\mathrm{CAR}^{+}(\mathfrak{h})$ invariant and $\left(\mathrm{CAR}^{+}(\mathfrak{h}), \tau^{t}\right)$ is a $C^{*}$-dynamical system which describes an ideal Fermi gas.

When the $C^{*}$-algebra $\mathfrak{A}$ does not act naturally on a Hilbert space, the situation is more involved and Banach space techniques must be used. I will only mention a simple example based on the powerful technique of analytic vectors (see Chapter 3.1 in [11], Section 5 in [32] and Chapter 6.2 in [12] for a more systematic exposition of these techniques).

Definition 9. Let $T$ be an operator on a Banach space $\mathcal{B}$. $A$ vector $x \in \mathcal{B}$ is called analytic for $T$ if $x \in \cap_{n \in \mathbb{N}} \mathrm{D}\left(T^{n}\right)$ and if there exists $\rho_{x}>0$ such that the power series

$$
\sum_{n=0}^{\infty} \frac{z^{n}}{n !}\left\|T^{n} x\right\|
$$

defines an analytic function in $\left\{z \in \mathbb{C}|| z \mid<\rho_{x}\right\}$.

Note that the set of analytic vectors of an operator $T$ is a subspace. If $x$ is analytic for $T$ one can define

$$
\mathrm{e}^{t T} x \equiv \sum_{n=0}^{\infty} \frac{t^{n}}{n !} T^{n} x
$$

for $|t|<\rho_{x}$. Moreover, if $T$ is closed and $t<\rho_{x}$ then it is easy to show that $\mathrm{e}^{t T} x$ is analytic for $T$ and a simple manipulation of norm convergent series yields that $\mathrm{e}^{s T} \mathrm{e}^{t T} x=\mathrm{e}^{(s+t) T} x$ as long as $|s|+|t|<\rho_{x}$. If the subspace of analytic vectors is dense in $\mathcal{B}$ and if one can prove that the linear operator $\mathrm{e}^{t T}$ defined in this way is bounded (this is usually done with the help of some dissipativity estimate), then it extends to all of $\mathcal{B}$ and using the group property its definition can be extended to all $t \in \mathbb{R}$. 
Example 3. (Quantum spin systems) Let $\Gamma$ be an infinite lattice (for example $\Gamma=\mathbb{Z}^{d}$, with $d \geqslant 1$ ) and to each $x \in \Gamma$ associate a copy $\mathfrak{h}_{x}$ of a finite dimensional Hilbert space $\mathfrak{h}$. For finite subsets $\Lambda \subset \Gamma$ set $\mathfrak{h}_{\Lambda} \equiv \otimes_{x \in \Lambda} \mathfrak{h}_{x}$ and define the local $C^{*}$-algebra

$$
\mathfrak{A}_{\Lambda} \equiv \mathfrak{B}\left(\mathfrak{h}_{\Lambda}\right) .
$$

If $\Lambda \subset \Lambda^{\prime}$, the natural injection $A \mapsto A \otimes I_{\mathfrak{h}_{\Lambda^{\prime} \backslash \Lambda}}$ allows to identify $\mathfrak{A}_{\Lambda}$ with a subalgebra of $\mathfrak{A}_{\Lambda^{\prime}}$. Therefore, a $C^{*}$-norm can be defined on $\mathfrak{A}_{\text {loc }} \equiv \cup_{\Lambda \subset \Gamma} \mathfrak{A}_{\Lambda}$, the union being over finite subsets of $\Gamma$. Denote by $\mathfrak{A}$ the $C^{*}$-algebra obtained as norm closure of $\mathfrak{A}_{\text {loc }}$. Each local algebra $\mathfrak{A}_{\Lambda}$ is identified with the corresponding subalgebra of $\mathfrak{A}$. $C^{*}$-algebras of this type are called uniformly hyperfinite (UHF). Interpreting $\mathfrak{h}$ as the Hilbert space of a single spin, $\mathfrak{A}$ describes the observables of a quantum spin system on the lattice $\Gamma$.

An interaction is a map $X \mapsto \phi(X)$ which, to any finite subset $X$ of the lattice $\Gamma$, associates a self-adjoint element $\phi(X)$ of $\mathfrak{A}_{X}$ describing the interaction energy of the degrees of freedom inside $X$. For example, in the spin interpretation, if $X=\{x\}$ then $\phi(X)$ is the energy of the spin at $x$ due to the coupling of its magnetic moment with an external magnetic field. If $X=\{x, y\}$ then $\phi(X)$ is the coupling energy due to the pair interaction between the corresponding magnetic moments.

Given an interaction $\phi$, the local Hamiltonian for a finite region $\Lambda \subset \Gamma$ is the self-adjoint element of $\mathfrak{A}_{\Lambda}$ given by

$$
H_{\Lambda} \equiv \sum_{X \subset \Lambda} \phi(X)
$$

and a $C^{*}$-dynamical system is defined on $\mathfrak{A}$ by

$$
\tau_{\Lambda}^{t}(A) \equiv \mathrm{e}^{i t H_{\Lambda}} A \mathrm{e}^{-i t H_{\Lambda}} .
$$

Assume that the interaction $\phi$ has sufficiently short range, more precisely that

$$
\|\phi\|_{\sigma} \equiv \sup _{x \in \Gamma} \sum_{X \ni x}\|\phi(X)\| \mathrm{e}^{2 \sigma|X|}<\infty
$$

for some $\sigma>0$, where $|X|$ denotes the cardinality of the subset $X$. We shall show that the limit

$$
\tau^{t}(A) \equiv \lim _{\Lambda \uparrow \Gamma} \tau_{\Lambda}^{t}(A)
$$

exists for all $A \in \mathfrak{A}, t \in \mathbb{R}$ and defines a strongly continuous group of $*-$ morphisms.

The generator of $\tau_{\Lambda}^{t}$ is the bounded derivation

$$
\delta_{\Lambda}(A)=i\left[H_{\Lambda}, A\right],
$$

hence we have a norm convergent expansion

$$
\tau_{\Lambda}^{t}(A)=\sum_{n=0}^{\infty} \frac{t^{n}}{n !} \delta_{\Lambda}^{n}(A)
$$


For $A \in \mathfrak{A}_{\Lambda_{0}}$, we can further write

$$
\delta_{\Lambda}^{n}(A)=\sum_{X_{1}, \cdots, X_{n} \subset \Lambda} i\left[\phi\left(X_{n}\right), i\left[\phi\left(X_{n-1}\right), \cdots, i\left[\phi\left(X_{1}\right), A\right] \cdots\right]\right] .
$$

Since local algebras corresponding to disjoint subsets of $\Gamma$ commute, this sum can be restricted by the condition $X_{j} \cap \Lambda_{j-1} \neq \varnothing$ with $\Lambda_{j-1}=\Lambda_{0} \cup X_{1} \cdots \cup$ $X_{j-1}$. We proceed to estimate the norm of $\delta_{\Lambda}^{n}(A)$

$$
\begin{aligned}
& \left\|\delta_{\Lambda}^{n}(A)\right\| \leqslant \sum_{x_{1} \in \Lambda_{0}} \sum_{X_{1} \ni x_{1}} \sum_{x_{2} \in \Lambda_{1}} \sum_{X_{2} \ni x_{2}} \cdots \sum_{x_{n} \in \Lambda_{n-1}} \sum_{X_{n} \ni x_{n}} 2^{n}\|A\| \prod_{i=1}^{n}\left\|\phi\left(X_{i}\right)\right\| \\
& \leqslant 2^{n}\|A\|\left|\Lambda_{0}\right| \sup _{x_{1}} \sum_{X_{1} \ni x_{1}}\left|\Lambda_{1}\right| \sup _{x_{2}} \sum_{X_{2} \ni x_{2}} \cdots\left|\Lambda_{n-1}\right| \sup _{x_{n}} \sum_{X_{n} \ni x_{n}} \prod_{i=1}^{n}\left\|\phi\left(X_{i}\right)\right\| \\
& \leqslant 2^{n}\|A\| \sup _{x_{1}, \cdots, x_{n}} \sum_{X_{1} \ni x_{1}} \cdots \sum_{X_{n} \ni x_{n}}\left(\left|\Lambda_{0}\right|+\left|X_{1}\right|+\cdots+\left|X_{n}\right|\right)^{n} \prod_{i=1}^{n}\left\|\phi\left(X_{i}\right)\right\| .
\end{aligned}
$$

Using the inequality $\mathrm{e}^{2 \sigma x} \geqslant(2 \sigma x)^{n} / n$ ! with $x=\left|\Lambda_{0}\right|+\left|X_{1}\right|+\cdots+\left|X_{n}\right|$ we finally get the following uniform estimate in $\Lambda$

$$
\left\|\delta_{\Lambda}^{n}(A)\right\| \leq \frac{n !}{\sigma^{n}} \mathrm{e}^{2 \sigma\left|\Lambda_{0}\right|}\|A\|\|\phi\|_{\sigma}^{n} .
$$

From this we conclude that

$$
\delta^{(n)}(A)=\lim _{\Lambda} \delta_{\Lambda}^{n}(A)=\sum_{X_{1}, \cdots, X_{n}} i\left[\phi\left(X_{n}\right), i\left[\phi\left(X_{n-1}\right), \cdots, i\left[\phi\left(X_{1}\right), A\right] \cdots\right]\right],
$$

exists and satisfies the same estimate (16). It is also clear from this argument that

$$
\lim _{\Lambda} \delta^{(n)}\left(\delta_{\Lambda}^{k}(A)\right)=\delta^{(n+k)}(A) .
$$

Introducing (16) into the expansion (15), we conclude that the limit

$$
\tau^{t}(A)=\lim _{\Lambda} \tau_{\Lambda}^{t}(A)=\sum_{n=0}^{\infty} \frac{t^{n}}{n !} \delta^{(n)}(A),
$$

exists for $|t|<\sigma /\|\phi\|_{\sigma}$.

Since $\tau_{\Lambda}^{t}$ is isometric from any local algebra $\mathfrak{A}_{\Lambda_{0}}$ into $\mathfrak{A}$, it follows that the limit $\tau^{t}$ is norm continuous and extends by continuity to all of $\mathfrak{A}$. Furthermore, as a norm limit of $*$-morphisms, $\tau^{t}$ is a $*$-morphism.

For $|s|+|t|<\sigma /\|\phi\|_{\sigma}$ and $A \in \mathfrak{A}_{\text {loc }}$ the continuity of $\tau^{t}$ yields

$$
\lim _{\Lambda} \tau^{t}\left(\tau_{\Lambda}^{s}(A)\right)=\tau^{t}\left(\tau^{s}(A)\right)
$$

while Equ. (17) and the expansions (15)(18) lead, after a simple manipulation to 


$$
\lim _{\Lambda} \tau^{t}\left(\tau_{\Lambda}^{s}(A)\right)=\tau^{t+s}(A)
$$

Thus $\tau^{t}$ satisfies the local group property, and in particular is a $*$-isomorphism. Finally $\tau^{t}$ can be extended to a group by setting $\tau^{n t_{0}+t}=\left(\tau^{t_{0}}\right)^{n} \circ \tau^{t}$ and relation (14) then extends to all $t \in \mathbb{R}$ and $A \in \mathfrak{A}$.

Example 4. (Continuous classical dynamical system) Let $M$ be a compact metric space and $\varphi_{t}$ a continuous dynamics on $M$. Then the space $C(M)$ of continuous functions on $M$ is a commutative $C^{*}$-algebra with a unit on which the map $\tau^{t}(f)=f \circ \varphi_{t}$ defines a strongly continuous group of $*$-automorphisms.

\section{$4.2 W^{*}$-Dynamical Systems}

We have seen in Example 1 that even the simplest quantum mechanical system leads to a dynamics which is not strongly continuous when its Hamiltonian is unbounded. Thus, the notion of $C^{*}$-dynamical system is too restrictive for our purposes and we need to consider weaker topologies on the algebra of observables.

Let $\mathfrak{M} \subset \mathfrak{B}(\mathcal{H})$ be a von Neumann algebra. A group of $*$-automorphisms of $\mathfrak{M}$ is $\sigma$-weakly continuous if for all $A \in \mathfrak{M}$ the map $t \mapsto \tau^{t}(A)$ is continuous in the $\sigma$-weak topology. This means that for any $A \in \mathfrak{M}$ and any trace class operator $T$ on $\mathcal{H}$ the map $t \mapsto \operatorname{Tr}\left(T \tau^{t}(A)\right)$ is continuous.

Definition 10. $A W^{*}$-dynamical system is a pair $\left(\mathfrak{M}, \tau^{t}\right)$ where $\mathfrak{M}$ is a von Neumann algebra acting on a Hilbert space $\mathcal{H}$ and $\tau^{t}$ is a $\sigma$-weakly continuous group of *-automorphisms of $\mathfrak{M}$.

Remark 5. More generally, $\mathfrak{M}$ could be an abstract $W^{*}$-algebra, i.e., a $C^{*}$ algebra which is the dual Banach space of a Banach space $\mathfrak{M}_{\star}$, and $\tau^{t}$ a weak* continuous group of $*$-automorphisms. Since by Sakai theorem (see [42], Theorem 1.16.7) a $W^{*}$-algebra is $*$-isomorphic to a von Neumann subalgebra of $\mathfrak{B}(\mathcal{H})$ for some Hilbert space $\mathcal{H}$, I will only consider this particular situation. The predual $\mathfrak{M}_{\star}$ is then canonically identified with the quotient $\mathcal{L}^{1}(\mathcal{H}) / \mathfrak{M}^{\perp}$ where $\mathcal{L}^{1}(\mathcal{H})$ is the Banach space of trace class operators on $\mathcal{H}$ and

$$
\mathfrak{M}^{\perp}=\left\{T \in \mathcal{L}^{1}(\mathcal{H}) \mid \operatorname{Tr}(T A)=0 \text { for all } A \in \mathfrak{M}\right\},
$$

the annihilator of $\mathfrak{M}$. The weak- $\star$ topology on $\mathfrak{M}$ induced by $\mathfrak{M}_{\star}$ coincides with the $\sigma$-weak topology and elements of the predual corresponds to $\sigma$-weakly continuous linear functionals on $\mathfrak{M}$.

We have seen in Subsection 2.1 (Corollary 1) that a $*$-automorphism of a von Neumann algebra is $\sigma$-weakly continuous. A $\sigma$-weakly continuous group of $\sigma$-weakly continuous linear operators is characterized by its generator, as in the strongly continuous case (see Chapter 3 in [11] for details). Thus, one can write $\tau^{t}=\mathrm{e}^{t \delta}$ and there is a characterization, parallel to Proposition 2, of $W^{*}$-dynamical systems (see Theorem 3.2.51 in [11]). 
Proposition 3. Let $\mathfrak{M}$ be a von Neumann algebra. A $\sigma$-weakly densely defined and closed linear operator $\delta$ on $\mathfrak{M}$ generates a $\sigma$-weakly continuous group of *-automorphisms of $\mathfrak{M}$ if and only if:

(i) $\delta$ is a $*$-derivation and $1 \in \mathrm{D}(\delta)$.

(ii) $\operatorname{Ran}(\operatorname{Id}+\lambda \delta)=\mathfrak{M}$ for all $\lambda \in \mathbb{R} \backslash\{0\}$.

(iii) $\|A+\lambda \delta(A)\| \geqslant\|A\|$ for all $\lambda \in \mathbb{R}$ and $A \in \mathrm{D}(\delta)$.

Example 5. (Finite quantum systems, continuation of Example 1) Consider now the case of an unbounded Hamiltonian $H$ on the Hilbert space $\mathcal{H}$. Clearly $\tau^{t}(A)=\mathrm{e}^{i t H} A \mathrm{e}^{-i t H}$ defines a group of $*$-automorphisms of the von Neumann algebra $\mathfrak{M}=\mathfrak{B}(\mathcal{H})$. For any unit vectors $\Phi, \Psi \in \mathcal{H}$ the function

$$
t \mapsto\left(\Phi, \tau^{t}(A) \Psi\right)=\left(\mathrm{e}^{-i t H} \Phi, A \mathrm{e}^{-i t H} \Psi\right)
$$

is continuous and uniformly bounded in $t$ by $\|A\|$. If $T$ is a trace class operator on $\mathcal{H}$ then one has $T=\sum_{n} t_{n}\left(\Phi_{n}, \cdot\right) \Psi_{n}$ with $\left\|\Psi_{n}\right\|=\left\|\Phi_{n}\right\|=1$ and $\sum_{n}\left|t_{n}\right|<$ $\infty$. It follows that

$$
t \mapsto \operatorname{Tr}\left(T \tau^{t}(A)\right)=\sum_{n} t_{n}\left(\Phi_{n}, \tau^{t}(A) \Psi_{n}\right),
$$

is continuous (as a uniformly convergent series of continuous functions). Thus $\tau^{t}$ is $\sigma$-weakly continuous and $\left(\mathfrak{M}, \tau^{t}\right)$ is a $W^{*}$-dynamical system.

Example 6. (Ideal Bose gas) Let $\mathfrak{h}$ be a Hilbert space and $\Gamma_{+}(\mathfrak{h})$ the Bosonic Fock space over $\mathfrak{h}$ (see Section 2 in Lecture [36]). For $f \in \mathfrak{h}$, denote by

$$
\Phi(f)=\frac{1}{\sqrt{2}}\left(a^{*}(f)+a(f)\right),
$$

the self-adjoint Segal field operator. Since $\Phi(f)$ is unbounded for $f \neq 0$, it is more convenient to use the unitary Weyl operators $W(f)=\mathrm{e}^{i \Phi(f)}$. They define a projective representation of the additive group $\mathfrak{h}$ on $\Gamma_{+}(\mathfrak{h})$ satisfying the Weyl relations

$$
W(f) W(g)=\mathrm{e}^{-i \operatorname{Im}(f, g) / 2} W(f+g) .
$$

To any self-adjoint operator $h$ on $\mathfrak{h}$ we can, as in the Fermionic case, associate the second quantized strongly continuous unitary group $U^{t}=\Gamma\left(\mathrm{e}^{i t h}\right)$ on $\Gamma_{+}(\mathfrak{h})$. The action of this group on Weyl operators is given by

$$
U^{t} W(f) U^{t *}=W\left(\mathrm{e}^{i t h} f\right) .
$$

It follows from Equ. (19) that, for $f \neq 0$ and $g=i \theta f /\|f\|^{2}$,

$$
W(g)^{*} W(f) W(g)=\mathrm{e}^{-i \theta} W(f) .
$$

This shows that the spectrum of $W(f)$ is the full unit circle and therefore 


$$
\|W(f)-W(g)\|=\left\|W(f-g)-\mathrm{e}^{-i \operatorname{Im}(f, g) / 2}\right\|=2,
$$

for $f \neq g$. This makes clear that in the Bosonic framework there is no chance to obtain a strongly continuous group from Equ. (20). On the other hand it follows from Exercise 5 that the von Neumann algebra

$$
\mathfrak{W}(\mathfrak{h})=\{W(f) \mid f \in \mathfrak{h}\}^{\prime \prime},
$$

together with the group

$$
\tau^{t}(A)=U^{t} A U^{t *}
$$

form a $W^{*}$-dynamical system.

Exercise 18. Show that the system $\{W(f) \mid f \in \mathfrak{h}\}$ is irreducible, i.e., that

$$
\{W(f) \mid f \in \mathfrak{h}\}^{\prime}=\mathbb{C} I .
$$

Conclude that $\mathfrak{W}(\mathfrak{h})=\mathfrak{B}\left(\Gamma_{+}(\mathfrak{h})\right)$. (Hint: see Subsection 2.4 in Lecture [36])

\subsection{Invariant States}

Definition 11. If $\tau^{t}$ is a group of *-automorphisms on a $C^{*}$-algebra $\mathfrak{A}$, a state $\mu$ on $\mathfrak{A}$ is called $\tau^{t}$-invariant if $\mu \circ \tau^{t}=\mu$ for all $t \in \mathbb{R}$. We denote by $E\left(\mathfrak{A}, \tau^{t}\right) \subset E(\mathfrak{A})$ the set of $\tau^{t}$-invariant states.

As in the case of classical dynamical systems, invariant states play a important role in the study of quantum dynamics.

Theorem 10. Let $\tau^{t}$ be a group of $*$-automorphisms of the $C^{*}$-algebra $\mathfrak{A}$. If there exists a state $\omega$ on $\mathfrak{A}$ such that the function $t \mapsto \omega\left(\tau^{t}(A)\right)$ is continuous for all $A \in \mathfrak{A}$ then $E\left(\mathfrak{A}, \tau^{t}\right)$ is a non-empty, convex and weak- $\star$ compact subset of $\mathfrak{A}^{\star}$. In particular, these conclusions hold if $\left(\mathfrak{A}, \tau^{t}\right)$ is a $C^{*}$ - or $W^{*}$-dynamical system.

Proof. To show that $E\left(\mathfrak{A}, \tau^{t}\right)$ is not empty, we follow the strategy of Exercise 10. For all $A \in \mathfrak{A}$ consider the expression

$$
\omega_{T}(A) \equiv \frac{1}{T} \int_{0}^{T} \omega \circ \tau^{s}(A) \mathrm{d} s .
$$

Since the function $s \mapsto \omega \circ \tau^{s}(A)$ is continuous, the integral is well defined and we clearly have $\omega_{T} \in E(\mathfrak{A})$ for all $T>0$. Since $E(\mathfrak{A})$ is weak- $\star$ compact, the net $\left(\omega_{T}\right)_{T>0}$ has a weak- $\star$ convergent subnet. The formula

$$
\omega_{T}\left(\tau^{t}(A)\right)=\omega_{T}(A)-\frac{1}{T} \int_{0}^{t} \omega \circ \tau^{t}(A) \mathrm{d} s+\frac{1}{T} \int_{T}^{T+t} \omega \circ \tau^{s}(A) \mathrm{d} s
$$

leads to the estimate 


$$
\left|\omega_{T}\left(\tau^{t}(A)\right)-\omega_{T}(A)\right| \leqslant 2\|A\| \frac{|t|}{T},
$$

from which it follows that the limit of any convergent subnet of $\left(\omega_{T}\right)_{T>0}$ is $\tau^{t}$-invariant.

It is clear that the set of $\tau^{t}$-invariant states is convex and weak- $\star$ closed.

Definition 12. If $\tau^{t}$ is a group of *-automorphisms of the von Neumann algebra $\mathfrak{M}$ we denote by $N\left(\mathfrak{M}, \tau^{t}\right) \equiv E\left(\mathfrak{M}, \tau^{t}\right) \cap N(\mathfrak{M})$ the set of normal $\tau^{t}$ invariant states.

Ii immediately follows from the last paragraph in Subsection 2.1 that if $\omega \in N\left(\mathfrak{M}, \tau^{t}\right)$ then $\tau^{t}\left(s_{\omega}\right)=s_{\omega}$ and $\tau^{t}\left(z_{\omega}\right)=z_{\omega}$.

We note that for a $W^{*}$-dynamical system $\left(\mathfrak{M}, \tau^{t}\right)$ the compactness argument used in the proof of Theorem 10 breaks down if we replace $E(\mathfrak{M})$ by $N(\mathfrak{M})$. There is no general existence result for normal invariant states of $W^{*}$-dynamical systems. In fact, there exists $W^{*}$-dynamical systems without normal invariant states (see Exercise 22). However, we shall see below that any invariant state of a $C^{*}$ - or $W^{*}$-dynamical system can be described as a normal invariant state of some associated $W^{*}$-dynamical system. For this reason, normal invariant states play an important role in quantum dynamics.

\subsection{Quantum Dynamical Systems}

Definition 13. If $\mathfrak{C}$ is a $C^{*}$-algebra and $\tau^{t}$ a group of $*$-automorphisms of $\mathfrak{C}$ we define

$$
\mathcal{E}\left(\mathfrak{C}, \tau^{t}\right) \equiv\left\{\mu \in E\left(\mathfrak{C}, \tau^{t}\right) \mid t \mapsto \mu\left(A^{*} \tau^{t}(A)\right) \text { is continuous for all } A \in \mathfrak{C}\right\} .
$$

If $\mu \in \mathcal{E}(\mathfrak{C}, \tau)$ we say that $\left(\mathfrak{C}, \tau^{t}, \mu\right)$ is a quantum dynamical system.

Example \%. If $\left(\mathfrak{A}, \tau^{t}\right)$ is a $C^{*}$-dynamical system then $\mathcal{E}(\mathfrak{A}, \tau)=E(\mathfrak{A}, \tau)$ and $\left(\mathfrak{A}, \tau^{t}, \mu\right)$ is a quantum dynamical system for any $\tau^{t}$-invariant state $\mu$.

Example 8. If $\left(\mathfrak{M}, \tau^{t}\right)$ is a $W^{*}$-dynamical system then $N(\mathfrak{A}, \tau) \subset \mathcal{E}(\mathfrak{A}, \tau)$ and $\left(\mathfrak{M}, \tau^{t}, \mu\right)$ is a quantum dynamical system for any $\tau^{t}$-invariant normal state $\mu$.

Exercise 19. Show that $\mathcal{E}\left(\mathfrak{C}, \tau^{t}\right)$ is a convex, norm closed subset of $E\left(\mathfrak{C}, \tau^{t}\right)$.

In this subsection we shall study the GNS representation of a quantum dynamical system. We start with the following extension of the GNS construction.

Lemma 12. Let $\left(\mathfrak{C}, \tau^{t}, \mu\right)$ be a quantum dynamical system and denote the GNS representation of $\mathfrak{C}$ associated to $\mu$ by $\left(\mathcal{H}_{\mu}, \pi_{\mu}, \Omega_{\mu}\right)$. Then there exists a unique self-adjoint operator $L_{\mu}$ on $\mathcal{H}_{\mu}$ such that 
(i) $\pi_{\mu}\left(\tau^{t}(A)\right)=\mathrm{e}^{i t L_{\mu}} \pi_{\mu}(A) \mathrm{e}^{-i t L_{\mu}}$ for all $A \in \mathfrak{C}$ and $t \in \mathbb{R}$.

(ii) $L_{\mu} \Omega_{\mu}=0$.

Proof. For fixed $t \in \mathbb{R}$ one easily checks that $\left(\mathcal{H}_{\mu}, \pi_{\mu} \circ \tau^{t}, \Omega_{\mu}\right)$ is a GNS representation of $\mathfrak{C}$ associated to $\mu$. By unicity of the GNS construction there exists a unitary operator $U_{\mu}^{t}$ on $\mathcal{H}_{\mu}$ such that, for any $A \in \mathfrak{C}$, one has

$$
U_{\mu}^{t} \pi_{\mu}(A) \Omega_{\mu}=\pi_{\mu}\left(\tau^{t}(A)\right) \Omega_{\mu}
$$

and in particular

$$
U_{\mu}^{t} \Omega_{\mu}=\Omega_{\mu}
$$

For $t, s \in \mathbb{R}$ we have

$$
U_{\mu}^{t} U_{\mu}^{s} \pi_{\mu}(A) \Omega_{\mu}=U_{\mu}^{t} \pi_{\mu}\left(\tau^{s}(A)\right) \Omega_{\mu}=\pi_{\mu}\left(\tau^{t+s}(A)\right) \Omega_{\mu}=U_{\mu}^{t+s} \pi_{\mu}(A) \Omega_{\mu},
$$

and the cyclic property of $\Omega_{\mu}$ yields that $U_{\mu}^{t}$ is a unitary group on $\mathcal{H}_{\mu}$. Using Equ. (7) it follows from the continuity of the map

$$
t \mapsto\left(\pi_{\mu}(A) \Omega_{\mu}, U_{\mu}^{t} \pi_{\mu}(A) \Omega_{\mu}\right)=\mu\left(A^{*} \tau^{t}(A)\right),
$$

that $U_{\mu}^{t}$ is a strongly continuous. By Stone theorem $U_{\mu}^{t}=\mathrm{e}^{i t L_{\mu}}$ for some self-adjoint operator $L_{\mu}$ and property (ii) follows from Equ. (23).

Finally, for $A, B \in \mathfrak{C}$ we get

$$
U_{\mu}^{t} \pi_{\mu}(A) \pi_{\mu}(B) \Omega_{\mu}=\pi_{\mu}\left(\tau^{t}(A)\right) \pi_{\mu}\left(\tau^{t}(B)\right) \Omega_{\mu}=\pi_{\mu}\left(\tau^{t}(A)\right) U_{\mu}^{t} \pi_{\mu}(B) \Omega_{\mu},
$$

and property (i) follows from the cyclic property of $\Omega_{\mu}$.

To prove the uniqueness of $L_{\mu}$ note that Equ. (22) uniquely determines $U_{\mu}^{t}$ and that conditions (i) and (ii) imply that $\mathrm{e}^{i t L_{\mu}}$ satisfies (22).

Recall from subsection 2.2 that $\hat{\mu}(A)=\left(\Omega_{\mu}, A \Omega_{\mu}\right)$ defines a centrally faithful normal state on the enveloping von Neumann algebra $\mathfrak{C}_{\mu}=\pi_{\mu}(\mathfrak{C})^{\prime \prime}$. Moreover, by property (i) of Lemma 12, the $\sigma$-weakly continuous group of *-automorphisms of $\mathfrak{B}\left(\mathcal{H}_{\mu}\right)$ defined by

$$
\hat{\tau}_{\mu}^{t}(A) \equiv \mathrm{e}^{i t L_{\mu}} A \mathrm{e}^{-i t L_{\mu}}
$$

leaves $\pi_{\mu}(\mathfrak{C})$ and hence its $\sigma$-weak closure $\mathfrak{C}_{\mu}$ invariant. Thus, $\left(\mathfrak{C}_{\mu}, \hat{\tau}_{\mu}^{t}\right)$ is a $W^{*}$-dynamical system. By property (ii) of Lemma $12, \hat{\mu}$ is $\hat{\tau}_{\mu}^{t}$-invariant.

Definition 14. A quantum dynamical system $\left(\mathfrak{C}, \tau^{t}, \mu\right)$ is in normal form if

(i) $\mathfrak{C}$ is a von Neumann algebra on a Hilbert space $\mathcal{H}$.

(ii) $\tau^{t}(A)=\mathrm{e}^{i t L} A \mathrm{e}^{-i t L}$ for some self-adjoint operator $L$ on $\mathcal{H}$.

(iii) $\mu(A)=(\Omega, A \Omega)$ for some unit vector $\Omega \in \mathcal{H}$.

(iv) $\overline{\mathfrak{C} \Omega}=\mathcal{H}$.

(v) $L \Omega=0$. 
We denote by $(\mathfrak{C}, \mathcal{H}, L, \Omega)$ such a system.

The above considerations show that to any quantum dynamical system $\left(\mathfrak{C}, \tau^{t}, \mu\right)$ we can associate a quantum dynamical system in normal form $\left(\mathfrak{C}_{\mu}, \mathcal{H}_{\mu}, L_{\mu}, \Omega_{\mu}\right)$.

Definition 15. $\left(\pi_{\mu}, \mathfrak{C}_{\mu}, \mathcal{H}_{\mu}, L_{\mu}, \Omega_{\mu}\right)$ is the normal form of the quantum dynamical system $\left(\mathfrak{C}, \tau^{t}, \mu\right)$. The operator $L_{\mu}$ is its $\mu$-Liouvillean.

The normal form of a quantum dynamical system is uniquely determined, up to unitary equivalence.

Definition 16. Two quantum dynamical systems $\left(\mathfrak{C}, \tau^{t}, \mu\right),\left(\mathfrak{D}, \sigma^{t}, \nu\right)$ are isomorphic if there exists a $*$-isomorphism $\phi: \mathfrak{C} \rightarrow \mathfrak{D}$ such that $\phi \circ \tau^{t}=\sigma^{t} \circ \phi$ for all $t \in \mathbb{R}$ and $\mu=\nu \circ \phi$.

Exercise 20. Show that two isomorphic quantum dynamical systems share the same normal forms.

Remark 6. If $\omega \in \mathcal{E}\left(\mathfrak{C}, \tau^{t}\right)$ and $\mu \in N(\mathfrak{C}, \omega) \cap E\left(\mathfrak{C}, \tau^{t}\right)$ then $\mu=\tilde{\mu} \circ \pi_{\omega}$ for some $\tilde{\mu} \in N\left(\mathfrak{C}_{\omega}\right)$ and therefore

$$
\mu\left(A^{*} \tau^{t}(A)\right)=\tilde{\mu}\left(\pi_{\omega}(A)^{*} \pi_{\omega}\left(\tau^{t}(A)\right)\right)=\tilde{\mu}\left(\pi_{\omega}(A)^{*} \mathrm{e}^{i t L_{\omega}} \pi_{\omega}(A) \mathrm{e}^{-i t L_{\omega}}\right),
$$

is a continuous function of $t \in \mathbb{R}$. It follows that $\mu \in \mathcal{E}\left(\mathfrak{C}, \tau^{t}\right)$. Denote by $\hat{\tau}_{\omega}^{t}$ the $W^{*}$-dynamics on $\mathfrak{C}_{\omega}$ generated by the $\omega$-Liouvillean $L_{\omega}$. Since $\mu$ is $\tau^{t}$ invariant one has $\tilde{\mu}\left(\hat{\tau}_{\omega}^{t}(A)\right)=\tilde{\mu}(A)$ for all $A \in \pi_{\omega}(\mathfrak{C})$ and by continuity $\tilde{\mu}$ is $\hat{\tau}_{\omega}^{t}$-invariant. Let $\pi_{\mu \mid \omega}: \mathfrak{C}_{\omega} \rightarrow \mathfrak{C}_{\mu}$ be the $*$-morphism of Theorem 4 . From the identity

$$
\begin{aligned}
\hat{\tau}_{\mu}^{t} \circ \pi_{\mu \mid \omega} \circ \pi_{\omega} & =\hat{\tau}_{\mu}^{t} \circ \pi_{\mu} \\
& =\pi_{\mu} \circ \tau^{t}=\pi_{\mu \mid \omega} \circ \pi_{\omega} \circ \tau^{t} \\
& =\pi_{\mu \mid \omega} \circ \hat{\tau}_{\omega}^{t} \circ \pi_{\omega},
\end{aligned}
$$

it follows by $\sigma$-weak continuity that

$$
\hat{\tau}_{\mu}^{t} \circ \pi_{\mu \mid \omega}=\pi_{\mu \mid \omega} \circ \hat{\tau}_{\omega}^{t},
$$

and since $z_{\mu \mid \omega}$ is invariant under $\hat{\tau}_{\omega}^{t}$

$$
\hat{\tau}_{\mu}^{t} \circ \hat{\pi}_{\mu \mid \omega}=\hat{\pi}_{\mu \mid \omega} \circ \hat{\tau}_{\omega}^{t} .
$$

We conclude that $\hat{\pi}_{\mu \mid \omega}$ is an isomorphism between the quantum dynamical systems $\left(z_{\mu \mid \omega} \mathfrak{C}_{\omega}, \hat{\tau}_{\omega}^{t}, \tilde{\mu}\right)$ and $\left(\mathfrak{C}_{\mu}, \hat{\tau}_{\mu}^{t}, \hat{\mu}\right)$.

The normal form turns out to be a very useful tool in the study of quantum dynamics since it provides a unifying framework in which both $C^{*}$ - and $W^{*}$ systems can be handled on an equal footing. 
Example 9. (Finite quantum systems) Let $\left(\mathfrak{M}, \tau^{t}\right)$ be the $C^{*}$ - or $W^{*}$-dynamical system constructed from the Hilbert space $\mathcal{H}$ and the Hamiltonian $H$, i.e., $\mathfrak{M} \equiv \mathfrak{B}(\mathcal{H})$ and $\tau^{t}(A) \equiv \mathrm{e}^{i t H} A \mathrm{e}^{-i t H}$ (Examples 1 and 5$)$. A density matrix $\rho$ on $\mathcal{H}$ such that $\mathrm{e}^{-i t H} \rho \mathrm{e}^{i t H}=\rho$ defines a normal, $\tau^{t}$-invariant state $\mu(A)=\operatorname{Tr}(\rho A)$. If $H$ has non-empty point spectrum, such states are easily obtained as mixtures of eigenstates of $H .\left(\mathfrak{M}, \tau^{t}, \mu\right)$ is a quantum dynamical system. Its normal form can be described in the following way. Set $\mathcal{G} \equiv \overline{\operatorname{Ran} \rho}$, denote by $\iota: \mathcal{G} \hookrightarrow \mathcal{H}$ the canonical injection and by $\mathcal{L}^{2}(\mathcal{G}, \mathcal{H})$ the set of Hilbert-Schmidt operators from $\mathcal{G}$ to $\mathcal{H}$. Then

$$
\mathcal{H}_{\mu} \equiv \mathcal{L}^{2}(\mathcal{G}, \mathcal{H})
$$

is a Hilbert space with inner product $(X, Y) \equiv \operatorname{Tr}\left(X^{*} Y\right)$. Since $P \equiv \iota \iota^{*}$ is the orthogonal projection of $\mathcal{H}$ on $\mathcal{G}$, a simple calculation shows that $\Omega_{\mu} \equiv \rho^{1 / 2} \iota$ is a unit vector in $\mathcal{H}_{\mu}$. Setting

$$
\pi_{\mu}(A) X=A X
$$

for all $X \in \mathcal{H}_{\mu}$, the map $A \mapsto \pi_{\mu}(A)$ defines a faithful representation of $\mathfrak{M}$ on $\mathcal{H}_{\mu}$. Since $\left(X, \pi_{\mu}(A) \Omega_{\mu}\right)=\operatorname{Tr}\left(\left(\rho^{1 / 2} \iota X^{*}\right) A\right)$, one immediately checks that $\pi_{\mu}(\mathfrak{M}) \Omega_{\mu}$ is dense in $\mathcal{H}_{\mu}$. Finally a simple calculation shows that for all $A \in \mathfrak{M}$ one has $\mu(A)=\left(\Omega_{\mu}, \pi_{\mu}(A) \Omega_{\mu}\right)$.

Since $\mu$ is normal and centrally faithful ${ }^{3}$ the enveloping von Neumann algebra is $\pi_{\mu}(\mathfrak{M})$ and $N(\mathfrak{M}, \mu)=N(\mathfrak{M})$. Let - denote an arbitrary complex conjugation on the Hilbert space $\mathcal{G}$. Then $\varphi \otimes \bar{\psi} \mapsto \varphi(\psi, \cdot)$ extends to a unitary map $U: \mathcal{H} \otimes \mathcal{G} \rightarrow \mathcal{H}_{\mu}$ such that $\pi_{\mu}(A)=U(A \otimes I) U^{*}$. Thus the enveloping von Neumann algebra $\pi_{\mu}(\mathfrak{M})$ is unitarily equivalent to $\mathfrak{M} \otimes I$.

Exercise 21. Show that the $\mu$-Liouvillean of the preceding example is given by

$$
\mathrm{e}^{i t L_{\mu}} X=\mathrm{e}^{i t H} X \mathrm{e}^{-i t H^{\prime}}
$$

where $H^{\prime}$ is the restriction of $H$ to $\mathcal{G}$. What is the spectrum of $L_{\mu}$ ?

Exercise 22. Show that if $H$ has purely continuous spectrum, there is no trace class operator commuting with $H$, hence no normal invariant state.

Example 10. (Ideal Bose gas, continuation of Example 6) Let $\mathcal{D} \subset \mathfrak{h}$ be a subspace and denote by $\operatorname{CCR}(\mathcal{D})$ the $C^{*}$-algebra generated by the Weyl system $W(\mathcal{D}) \equiv\{W(f) \mid f \in \mathcal{D}\}$, i.e., the norm closure of the linear span of $W(\mathcal{D})$. Since we can replace $\mathfrak{h}$ by $\overline{\mathcal{D}}$ we may assume, without loss of generality, that $\mathcal{D}$ is dense in $\mathfrak{h}$.

Let $\mathcal{H}$ be a Hilbert space and $\pi: W(\mathcal{D}) \rightarrow \mathfrak{B}(\mathcal{H})$ be such that $\pi(W(f))$ is unitary and

$$
\pi(W(f)) \pi(W(g))=\mathrm{e}^{-i \operatorname{Im}(f, g) / 2} \pi(W(f+g)),
$$

${ }^{3}$ Because $\mathfrak{M}$ is a factor. 
for all $f, g \in \mathcal{D}$. Then one can show (see Theorem 6 in Lecture [36]) that $\pi$ has a unique extension to an injective $*$-morphism from $\operatorname{CCR}(\mathcal{D})$ into $\mathfrak{B}(\mathcal{H})$. Thus, a representation of $\operatorname{CCR}(\mathcal{D})$ is completely determined by its restriction to $W(\mathcal{D})$.

A representation $(\mathcal{H}, \pi)$ of $\operatorname{CCR}(\mathcal{D})$ is regular if the map $\lambda \mapsto \pi(W(\lambda f))$ is strongly continuous for all $f \in \mathcal{D}$. Regular representations are physically appealing since by Stone theorem there exists a self-adjoint operator $\Phi_{\pi}(f)$ on $\mathcal{H}$ such that $\pi(W(f))=\mathrm{e}^{i \Phi_{\pi}(f)}$ for all $f \in \mathcal{D}$. The operator $\Phi_{\pi}(f)$ is the Segal field operator in the representation $\pi$. The corresponding creation and annihilation operators are obtained by linear combination, for example $a_{\pi}(f)=\left(\Phi_{\pi}(f)+i \Phi_{\pi}(i f)\right) / \sqrt{2}$. A state $\omega$ on $\operatorname{CCR}(\mathcal{D})$ is called regular if its GNS representation is regular.

Since the finite linear combinations of elements of $W(\mathcal{D})$ are norm dense in $\operatorname{CCR}(\mathcal{D})$, a state $\omega$ on this $C^{*}$-algebra is completely determined by its characteristic function

$$
\mathcal{D} \ni f \mapsto S_{\omega}(f) \equiv \omega(W(f)) .
$$

Clearly, if the function $\lambda \mapsto S_{\omega}(\lambda f)$ is continuous for all $f \in \mathcal{D}$, the state $\omega$ is regular and we denote by $\Phi_{\omega}(f), a_{\omega}(f)$ and $a_{\omega}^{*}(f)$ the corresponding operators. The state $\omega$ is said to be $C^{n}, C^{\infty}$ respectively analytic if the function $\lambda \mapsto$ $S_{\omega}(\lambda f)$ has this smoothness near $\lambda=0$. If $\omega$ is an analytic state, it is easy to see that $S_{\omega}(\lambda f)$ is actually analytic in an open strip around the real axis and that $\Omega_{\omega}$ is an analytic vector for all field operators $\Phi_{\omega}(f)$. The characteristic function $S_{\omega}(f)$ and therefore the state $\omega$ itself are then completely determined by the derivatives $\left.\partial_{\lambda}^{n} S_{\omega}(\lambda f)\right|_{\lambda=0}$, or equivalently by the family of correlation functions

$$
W_{m, n}\left(g_{1}, \cdots, g_{m} ; f_{1}, \cdots, f_{n}\right)=\left(\Omega_{\omega}, a_{\omega}^{*}\left(g_{m}\right) \cdots a_{\omega}^{*}\left(g_{1}\right) a_{\omega}\left(f_{1}\right) \cdots a_{\omega}\left(f_{n}\right) \Omega_{\omega}\right) .
$$

Characteristic functions of regular states on $\operatorname{CCR}(\mathcal{D})$ are characterized by the the following result (see Lecture [36] for a proof).

Theorem 11. (Araki-Segal) $A$ map $S: \mathcal{D} \rightarrow \mathbb{C}$ is the characteristic function of a regular state $\omega$ on $\operatorname{CCR}(\mathcal{D})$ if and only if

(i) $S(0)=1$.

(ii) The function $\lambda \mapsto S(\lambda f)$ is continuous for all $f \in \mathcal{D}$.

(iii) For all integer $n \geqslant 2$, all $f_{1}, \cdots, f_{n} \in \mathcal{D}$ and all $z_{1}, \cdots, z_{n} \in \mathbb{C}$ one has

$$
\sum_{j, k=1}^{n} S\left(f_{j}-f_{k}\right) \mathrm{e}^{-i \operatorname{Im}\left(f_{j}, f_{k}\right) / 2} \bar{z}_{j} z_{k} \geqslant 0 .
$$

If the subspace $\mathcal{D}$ is invariant under the one-particle dynamics, i.e., if $\mathrm{e}^{-i t h} \mathcal{D} \subset \mathcal{D}$ for all $t \in \mathbb{R}$, then it follows from Equ. (20) that the group $\tau^{t}$ defined by Equ. (21) leaves $W(\mathcal{D})$ and hence its closed linear span $\operatorname{CCR}(\mathcal{D})$ invariant. Thus, $\tau^{t}$ is a group of $*$-automorphisms of $\operatorname{CCR}(\mathcal{D})$. Note that 
$\left(\operatorname{CCR}(\mathcal{D}), \tau^{t}\right)$ is neither a $C^{*}$-dynamical $\left(\tau^{t}\right.$ is not strongly continuous by Example 6), nor a $W^{*}$-dynamical system $(\operatorname{CCR}(\mathcal{D})$ is not a von Neumann algebra).

Suppose that the map $S: \mathcal{D} \rightarrow \mathbb{C}$ satisfies the conditions of Theorem 11 and

(iv) $S\left(\mathrm{e}^{i t h} f\right)=S(f)$ for all $f \in \mathcal{D}$ and $t \in \mathbb{R}$.

(v) $\lim _{t \rightarrow 0} S\left(\mathrm{e}^{i t h} f-f\right)=1$ for all $f \in \mathcal{D}$.

Then the corresponding regular state $\omega$ is $\tau^{t}$-invariant and

$$
\omega\left(W(f)^{*} \tau^{t}(W(f))\right)=S\left(\mathrm{e}^{i t h} f-f\right),
$$

is continuous at $t=0$. By the Cauchy-Schwarz inequality the same is true of $\omega\left(W(g)^{*} \tau^{t}(W(f))\right)$ for any $f, g \in \mathcal{D}$. Since the linear span of $W(\mathcal{D})$ is norm dense in $\operatorname{CCR}(\mathcal{D})$ the group property of $\tau^{t}$ allows to conclude that for all $A \in \operatorname{CCR}(\mathcal{D})$ the function $t \mapsto \omega\left(A^{*} \tau^{t}(A)\right)$ is continuous. Therefore, $\omega \in \mathcal{E}\left(\operatorname{CCR}(\mathcal{D}), \tau^{t}\right)$ and $\left(\operatorname{CCR}(\mathcal{D}), \tau^{t}, \omega\right)$ is a quantum dynamical system.

Definition 17. A state on $\operatorname{CCR}(\mathcal{D})$ is called quasi-free if its characteristic function takes the form

$$
S(f)=\mathrm{e}^{-\frac{1}{4}\|f\|^{2}-\frac{1}{2} \rho[f]},
$$

where $\rho$ is a non-negative quadratic form on $\mathcal{D}$.

We shall denote by $\omega_{\rho}$ the quasi-free state characterized by Equ. (28). We shall also use the symbol $\rho$ to denote the non-negative self-adjoint operator associated with the quadratic form $\rho$. Thus, one has $\mathcal{D} \subset \mathrm{D}\left(\rho^{1 / 2}\right)$ and $\rho[f]=$ $\left\|\rho^{1 / 2} f\right\|^{2}$ for $f \in \mathcal{D}$.

The quasi-free state $\omega_{\rho}$ is clearly regular and analytic. If $\mathrm{e}^{-i t h} \rho \mathrm{e}^{i t h}=\rho$ for all $t \in \mathbb{R}$ then condition (iv) is satisfied and $\omega_{\rho}$ is $\tau^{t}$-invariant. Moreover, since

$$
\rho\left[\mathrm{e}^{i t h} f-f\right]=4\left\|\sin (t h / 2) \rho^{1 / 2} f\right\|^{2}
$$

condition (v) is also satisfied and $\omega_{\rho} \in \mathcal{E}\left(\operatorname{CCR}(\mathcal{D}), \tau^{t}\right)$.

To describe the normal form of $\left(\operatorname{CCR}(\mathcal{D}), \tau^{t}, \omega_{\rho}\right)$ let $\mathfrak{g} \equiv \overline{\operatorname{Ran} \rho} \subset \mathfrak{h}$, denote by $\iota: \mathfrak{g} \hookrightarrow \mathfrak{h}$ the canonical injection and set

$$
\mathcal{H}_{\omega_{\rho}} \equiv \mathcal{L}^{2}\left(\Gamma_{+}(\mathfrak{g}), \Gamma_{+}(\mathfrak{h})\right)
$$

the set of Hilbert-Schmidt operators from $\Gamma_{+}(\mathfrak{g})$ to $\Gamma_{+}(\mathfrak{h})$ with scalar product $(X, Y) \equiv \operatorname{Tr}\left(X^{*} Y\right)$. For $g \in \mathfrak{g}$ denote by $W^{\prime}(g)$ the Weyl operator in $\Gamma_{+}(\mathfrak{g})$.

For $f \in \mathcal{D}$ define

$$
\pi_{\omega_{\rho}}(W(f)): X \mapsto W\left((I+\rho)^{1 / 2} f\right) X W^{\prime}\left(\iota^{*} \rho^{1 / 2} f\right)^{*}
$$

Using the CCR (19), one easily checks that $\pi_{\omega_{\rho}}(W(f))$ is unitary and satisfies Equ. (27). Thus, $\pi_{\omega_{\rho}}$ has a unique extension to a representation of $\operatorname{CCR}(\mathcal{D})$ in $\mathcal{H}_{\omega_{\rho}}$. Denote by $\Omega, \Omega^{\prime}$ the Fock vacua in $\Gamma_{+}(\mathfrak{h}), \Gamma_{+}(\mathfrak{g})$ and set $\Omega_{\omega_{\rho}}=\Omega\left(\Omega^{\prime}, \cdot\right)$. 
Exercise 23. Show that $\Omega_{\omega_{\rho}}$ is cyclic for $\pi_{\omega_{\rho}}(\mathfrak{C})$. (Hint: For $X=\Psi(\Phi, \cdot)$ with $\Psi=a^{*}\left(f_{n}\right) \cdots a^{*}\left(f_{1}\right) \Omega$ and $\Phi=a^{* *}\left(g_{m}\right) \cdots a^{\prime *}\left(g_{1}\right) \Omega^{\prime}$ and $f \in \mathfrak{h}$ compute $\left.\partial_{\lambda} \pi_{\omega_{\rho}}(W(\lambda f)) X\right|_{\lambda=0}$ and $\left.\left.\partial_{\lambda} \pi_{\omega_{\rho}}(W(i \lambda f)) X\right|_{\lambda=0}.\right)$

A simple calculation shows that

$$
\omega_{\rho}(W(f))=\left(\Omega_{\omega_{\rho}}, \pi_{\omega_{\rho}}(W(f)) \Omega_{\omega_{\rho}}\right) .
$$

Finally, the $\omega_{\rho}$-Liouvillean is given by

$$
\mathrm{e}^{i t L_{\omega_{\rho}}} X \equiv \Gamma\left(\mathrm{e}^{i t h}\right) X \Gamma\left(\mathrm{e}^{-i t h^{\prime}}\right),
$$

where $h^{\prime}$ is the restriction of $h$ to $\mathfrak{g}$. The representation of $\operatorname{CCR}(\mathcal{D})$ obtained in this way is called Araki-Woods representation [1]. We refer the reader to [36] for a more detailed discussion and to [18] for a thorough exposition of the representation theory of canonical commutation relations.

\subsection{Standard Forms}

Recall that a subset $\mathcal{C}$ of a Hilbert space $\mathcal{H}$ is a cone if $t \psi \in \mathcal{C}$ for all $t \geq 0$ and $\psi \in \mathcal{C}$. The dual of a cone $\mathcal{C}$ is the closed cone

$$
\widehat{\mathcal{C}} \equiv\{\psi \in \mathcal{H} \mid(\phi, \psi) \geq 0 \text { for any } \phi \in \mathcal{C}\} .
$$

A cone $\mathcal{C}$ is self-dual if $\widehat{\mathcal{C}}=\mathcal{C}$. A self-dual cone is automatically closed.

Definition 18. A von Neumann algebra $\mathfrak{M} \subset \mathfrak{B}(\mathcal{H})$ is said to be in standard form if there exist a anti-unitary involution $J$ on $\mathcal{H}$ and a self-dual cone $\mathcal{C} \subset \mathcal{H}$ such that:

(i) $J \mathfrak{M} J=\mathfrak{M}^{\prime}$.

(ii) $J \Psi=\Psi$ for all $\Psi \in \mathcal{C}$.

(iii) $A J A \mathcal{C} \subset \mathcal{C}$ for all $A \in \mathfrak{M}$.

(iv) $J A J=A^{*}$ for all $A \in \mathfrak{M} \cap \mathfrak{M}^{\prime}$.

We shall denote by $(\mathfrak{M}, \mathcal{H}, J, \mathcal{C})$ a von Neumann algebra in standard form.

Theorem 12. Any von Neumann algebra $\mathfrak{M}$ has a faithful representation $(\mathcal{H}, \pi)$ such that $\pi(\mathfrak{M})$ is in standard form. Moreover, this representation is unique up to unitary equivalence.

Sketch of the proof. If $\mathfrak{M}$ is separable, i.e., if any family of mutually orthogonal projections in $\mathfrak{M}$ is finite or countable then $\mathfrak{M}$ has a normal faithful state $\omega$ (Proposition 2.5.6 in [11]). The associated GNS construction provides a faithful representation $\left(\mathcal{H}_{\omega}, \pi_{\omega}, \Omega_{\omega}\right)$ of $\mathfrak{M}$ and it follows from Tomita-Takesaki theory (Chapter 4 in Lecture [7]) that $\pi_{\omega}(\mathfrak{M})$ is in standard form. The antiunitary involution $J$ is the modular conjugation and the self-dual cone $\mathcal{C}$ is the natural cone $\overline{\left\{A J A \Omega_{\omega} \mid A \in \mathfrak{M}_{\omega}\right\}}$ (see Section 4.3 in Lecture [7]). This 
construction applies in particular to any von Neumann algebra over a separable Hilbert space which is the case most often encountered in physical applications.

In the general case, the construction is similar to the above one, substituting faithful normal states with faithful normal semi-finite weights. The general theory of standard forms was developed by Haagerup [23] following the works of Araki [3] and Connes [14] (see also [45], where standard forms are called hyper-standard).

Von Neumann algebras in standard form have two important properties which are of crucial importance in the study of quantum dynamical systems. The first one concerns normal states.

Theorem 13. Let $(\mathfrak{M}, \mathcal{H}, J, \mathcal{C})$ be a von Neumann algebra in standard form and for any unit vector $\Psi \in \mathcal{H}$ denote by $\omega_{\Psi} \in N(\mathfrak{M})$ the corresponding vector state $\omega_{\Psi}(A)=(\Psi, A \Psi)$. Then the map

$$
\begin{aligned}
\{\Phi \in \mathcal{C} \mid\|\Phi\|=1\} & \rightarrow N(\mathfrak{M}) \\
\Psi & \mapsto \omega_{\Psi}
\end{aligned}
$$

is an homeomorphism (for the norm topologies). In particular, for any normal state $\nu$ on $\mathfrak{M}$, there is a unique unit vector $\Psi_{\nu} \in \mathcal{C}$ such that $\nu=\omega_{\Psi_{\nu}} . W e$ call $\Psi_{\nu}$ the standard vector representative of $\nu$. Moreover, for any unit vectors $\Psi, \Phi \in \mathcal{C}$ one has:

(i) $\|\Psi-\Phi\|^{2} \leqslant\left\|\omega_{\Psi}-\omega_{\Phi}\right\| \leqslant\|\Psi-\Phi\|\|\Psi+\Phi\|$.

(ii) $\Psi$ is cyclic for $\mathfrak{M} \Longleftrightarrow \Psi$ is cyclic for $\mathfrak{M}^{\prime} \Longleftrightarrow \omega_{\Psi}$ is faithful.

(iii) More generally $\overline{\mathfrak{M} \Psi}=J \overline{\mathfrak{M}^{\prime} \Psi}$.

Proof. I shall not prove the fact that $\Psi \mapsto \omega_{\Psi}$ is an homeomorphism and the first inequality in (i) since this requires rather long and involved arguments. A proof can be found for example in [11]. The Second inequality in (i) follows from the polarization identity. The first equivalence in (ii) is a special case of (iii) which is a direct consequence of (i) and (ii) in Definition 18. The second equivalence in (ii) is a special case of the last statement of Lemma 3.

The second important property of von Neumann algebras in standard form has to do with the unitary implementation of $*$-automorphisms. To formulate this property let me introduce the following definition.

Definition 19. Let $(\mathfrak{M}, \mathcal{H}, J, \mathcal{C})$ be a von Neumann algebra in standard form. A unitary operator $U$ on $\mathcal{H}$ is called standard if the following holds:

(i) $U \mathcal{C} \subset \mathcal{C}$.

(ii) $U^{*} \mathfrak{M} U=\mathfrak{M}$. 
Obviously, the set of standard unitaries form a subgroup of the unitary group of $\mathcal{H}$. It is not hard to see that this subgroup is closed in the strong topology of $\mathfrak{B}(\mathcal{H})$. The following is essentially a rewriting of Corollary 4 in Lecture [7].

Theorem 14. Let $(\mathfrak{M}, \mathcal{H}, J, \mathcal{C})$ be a von Neumann algebra in standard form. Denote by $\mathcal{U}_{s}$ the group of standard unitaries of $\mathcal{H}$ equipped with the strong topology and by Aut $(\mathfrak{M})$ the group of *-automorphisms of $\mathfrak{M}$ with the topology of pointwise $\sigma$-weak convergence.

For any $U \in \mathcal{U}_{s}$ denote by $\tau_{U}$ the corresponding spatial $*$-automorphism $\tau_{U}(A)=U A U^{*}$. Then the map

$$
\begin{aligned}
\mathcal{U}_{s} & \rightarrow \operatorname{Aut}(\mathfrak{M}) \\
U & \mapsto \tau_{U}
\end{aligned}
$$

is an homeomorphism. In particular, for any *-automorphism $\sigma$ of $\mathfrak{M}$ there is a unique standard unitary $U$ such that $\sigma=\tau_{U}$. We call $U$ the standard implementation of $\sigma$. Moreover, for any $U \in \mathcal{U}_{s}$ one has:

(i) $[U, J]=0$.

(ii) $U \mathfrak{M}^{\prime} U^{*}=\mathfrak{M}^{\prime}$

(iii) $U^{*} \Psi_{\omega}=\Psi_{\omega \circ \tau_{U}}$ for all $\omega \in N(\mathfrak{M})$.

Definition 20. A quantum dynamical system $\left(\mathfrak{C}, \tau^{t}, \mu\right)$ is in standard form if

(i) $\mathfrak{C}$ is a von Neumann algebra in standard form $(\mathfrak{C}, \mathcal{H}, J, \mathcal{C})$.

(ii) $\left(\mathfrak{C}, \tau^{t}\right)$ is a $W^{*}$-dynamical system.

(iii) $\mu \in N\left(\mathfrak{C}, \tau^{t}\right)$.

Suppose that $\left(\mathfrak{C}, \tau^{t}, \mu\right)$ is in standard form. By Theorem 14, $\tau^{t}$ has a standard implementation $U^{t}$ for each $t \in \mathbb{R}$. Since $t \mapsto \tau^{t}(A)$ is $\sigma$-weakly continuous, $U^{t}$ is strongly continuous. Therefore, there exists a self-adjoint operator $L$ on $\mathcal{H}$ such that $U^{t}=\mathrm{e}^{i t L}$. By Theorem 13, there exists a unique vector $\Phi \in \mathcal{C}$ such that $\mu(A)=(\Phi, A \Phi)$. Moreover, it follows from properties (i)-(iii) of Theorem 14 that

$$
\begin{aligned}
J L+L J & =0 \\
\mathrm{e}^{i t L} \mathfrak{C}^{\prime} \mathrm{e}^{-i t L} & =\mathfrak{C}^{\prime}, \\
L \Psi_{\omega} & =0,
\end{aligned}
$$

for all $t \in \mathbb{R}$ and all $\omega \in N\left(\mathfrak{C}, \tau^{t}\right)$.

Definition 21. We denote by $(\mathfrak{C}, \mathcal{H}, J, \mathcal{C}, L, \Phi)$ a quantum dynamical system in standard form and we call $L$ its standard Liouvillean.

As an immediate consequence of this definition we have the 
Proposition 4. If the quantum dynamical system $\left(\mathfrak{C}, \tau^{t}, \mu\right)$ is in standard form $(\mathfrak{C}, \mathcal{H}, J, \mathcal{C}, L, \Phi)$, its standard Liouvillean is the unique self-adjoint operator $L$ on $\mathcal{H}$ such that, for all $t \in \mathbb{R}$ and all $A \in \mathfrak{C}$ one has

(i) $\mathrm{e}^{-i t L} \mathcal{C} \subset \mathcal{C}$

(ii) $\mathrm{e}^{i t L} A \mathrm{e}^{-i t L}=\tau^{t}(A)$.

Let $\left(\mathfrak{C}, \tau^{t}, \mu\right)$ be a quantum dynamical system, $\left(\pi_{\mu}, \mathfrak{C}_{\mu}, \mathcal{H}_{\mu}, L_{\mu}, \Omega_{\mu}\right)$ its normal form and $(\mathcal{H}, \pi)$ a standard representation of $\mathfrak{C}_{\mu}$. Then $\mathfrak{M} \equiv \pi\left(\mathfrak{C}_{\mu}\right)$ is a von Neumann algebra in standard form $(\mathfrak{M}, \mathcal{H}, J, \mathcal{C})$. Note that since $\pi$ is faithful it is $\sigma$-weakly continuous by Corollary 1 . The same remark apply to its inverse $\pi^{-1}: \mathfrak{M} \rightarrow \mathfrak{C}_{\mu}$.

Let $\eta \equiv \pi \circ \pi_{\mu}$ be the induced representation of $\mathfrak{C}$ in $\mathcal{H}$. Since $\pi_{\mu}(\mathfrak{C})$ is $\sigma$-weakly dense in $\mathfrak{C}_{\mu}$ we have $\mathfrak{M}=\eta(\mathfrak{C})^{\prime \prime}$. For $A \in \mathfrak{M}$ we define

$$
\sigma^{t}(A) \equiv \pi\left(\mathrm{e}^{i t L_{\mu}} \pi^{-1}(A) \mathrm{e}^{-i t L_{\mu}}\right)
$$

It follows that for $A \in \mathfrak{C}$ one has

$$
\begin{aligned}
\sigma^{t}(\eta(A)) & =\pi\left(\mathrm{e}^{i t L_{\mu}} \pi^{-1}(\eta(A)) \mathrm{e}^{-i t L_{\mu}}\right) \\
& =\pi\left(\mathrm{e}^{i t L_{\mu}} \pi_{\mu}(A) \mathrm{e}^{-i t L_{\mu}}\right) \\
& =\pi\left(\pi_{\mu}\left(\tau^{t}(A)\right)\right) \\
& =\eta\left(\tau^{t}(A)\right) .
\end{aligned}
$$

The group $\sigma^{t}$ defines a $W^{*}$-dynamical system on $\mathfrak{M}$. It has a standard implementation with standard Liouvillean $L$. Finally we remark that $\omega \equiv$ $\left(\Omega_{\mu}, \pi^{-1}(\cdot) \Omega_{\mu}\right) \in N\left(\mathfrak{M}, \sigma^{t}\right)$ satisfies $\omega \circ \eta=\mu$. Denote by $\Phi \in \mathcal{C}$ its standard vector representative. It follows that $\left(\mathfrak{M}, \sigma^{t}, \omega\right)$ is in standard form.

Definition 22. We say that $(\eta, \mathfrak{M}, \mathcal{H}, J, \mathcal{C}, L, \Phi)$ is the standard form of the quantum dynamical system $\left(\mathfrak{C}, \tau^{t}, \mu\right)$ and that $L$ is its standard Liouvillean.

If $\left(\pi_{1}, \mathfrak{D}_{1}, \mathcal{K}_{1}, M_{1}, \Omega_{1}\right)$ and $\left(\pi_{2}, \mathfrak{D}_{2}, \mathcal{K}_{2}, M_{2}, \Omega_{2}\right)$ are two normal forms of the quantum dynamical system $\left(\mathfrak{C}, \tau^{t}, \mu\right)$ then, by the unicity of the GNS representation, there exists a unitary $U: \mathcal{K}_{1} \rightarrow \mathcal{K}_{2}$ such that $\pi_{2}=U \pi_{1} U^{*}$, $\Omega_{2}=U \Omega_{1}$ and $M_{2}=U M_{1} U^{*}$. Let $\left(\eta_{1}, \mathcal{H}_{1}\right)$ and $\left(\eta_{2}, \mathcal{H}_{2}\right)$ be standard representations of $\mathfrak{D}_{1}$ and $\mathfrak{D}_{2}$ and denote by $\left(\eta_{1} \circ \pi_{1}, \mathfrak{M}_{1}, \mathcal{H}_{1}, J_{1}, \mathcal{C}_{1}, L_{1}, \Phi_{1}\right)$ and $\left(\eta_{2} \circ \pi_{2}, \mathfrak{M}_{2}, \mathcal{H}_{2}, J_{2}, \mathcal{C}_{2}, L_{2}, \Phi_{2}\right)$ the corresponding standard forms of $\left(\mathfrak{C}, \tau^{t}, \mu\right)$. Since $\left(\eta_{2}\left(U \cdot U^{*}\right), \mathcal{H}_{2}\right)$ is another standard representation of $\mathfrak{D}_{1}$, there exists a unitary $V: \mathcal{H}_{1} \rightarrow \mathcal{H}_{2}$ such that $\eta_{2}\left(U A U^{*}\right)=V \eta_{1}(A) V^{*}$. It follows that $\eta_{2} \circ \pi_{2}=V \eta_{1}\left(U^{*} \pi_{2} U\right) V^{*}=V \eta_{1} \circ \pi_{1} V^{*}$. Thus, the standard form and in particular the standard Liouvillean of a quantum dynamical system are uniquely determined, up to unitary equivalence.

The next proposition elucidate the relation between the $\mu$-Liouvillean and the standard Liouvillean. It also shows that in many applications (see Lemma 12 below) the two coincide. 
Proposition 5. Let $\left(\mathfrak{C}, \tau^{t}, \mu\right)$ be a quantum dynamical system with normal form $\left(\pi_{\mu}, \mathfrak{C}_{\mu}, \mathcal{H}_{\mu}, L_{\mu}, \Omega_{\mu}\right)$. If the state $\hat{\mu} \equiv\left(\Omega_{\mu},(\cdot) \Omega_{\mu}\right)$ is faithful on $\mathfrak{C}_{\mu}$ then this von Neumann algebra is in standard form $\left(\mathfrak{C}_{\mu}, \mathcal{H}_{\mu}, J, \mathcal{C}\right)$ and the standard form of $\left(\mathfrak{C}, \tau^{t}, \mu\right)$ is given by $\left(\pi_{\mu}, \mathfrak{C}_{\mu}, \mathcal{H}_{\mu}, J, \mathcal{C}, L_{\mu}, \Omega_{\mu}\right)$. In particular $L_{\mu}$ is its standard Liouvillean.

Proof. If $\hat{\mu}$ is faithful then, as mentioned in the sketch of the proof of Theorem $12, \mathfrak{C}_{\mu}$ is in standard form. $J$ is the modular conjugation associated to $\Omega_{\mu}$ and $\mathcal{C}$ is the associated natural cone. In particular, $\Omega_{\mu}$ is the standard vector representative of $\hat{\mu}$. If $L$ is the standard Liouvillean then $L \Omega_{\mu}=0$ by Equ. (31) hence $L$ is the $\mu$-Liouvillean.

More generally, the relation between $\mu$-Liouvillean and standard Liouvillean is given by the following result.

Proposition 6. Let $\left(\mathfrak{C}, \tau^{t}, \mu\right)$ be a quantum dynamical system with standard form $(\eta, \mathfrak{M}, \mathcal{H}, J, \mathcal{C}, L, \Phi)$. Then the subspace $\mathcal{K} \equiv \overline{\mathfrak{M} \Phi} \subset \mathcal{H}$ reduces the standard Liouvillean $L$ and the $\mu$-Liouvillean $L_{\mu}$ is (unitarily equivalent to) the restriction of $L$ to $\mathcal{K}$. In particular, one has $\sigma\left(L_{\mu}\right) \subset \sigma(L)$.

Proof. We reconstruct a normal form of $\left(\mathfrak{C}, \tau^{t}, \mu\right)$ out of its standard form. Recall that $\eta: \mathfrak{C} \rightarrow \mathfrak{M}$ is a representation such that $\mathfrak{M}=\eta(\mathfrak{C})^{\prime \prime}, \mu(A)=$ $(\Phi, \eta(A) \Phi)$ and $\eta\left(\tau^{t}(A)\right)=\mathrm{e}^{i t L} \eta(A) \mathrm{e}^{-i t L}$. Denote by $k: \mathcal{K} \hookrightarrow \mathcal{H}$ the canonical injection. Since $\mathcal{K}$ is invariant under $\mathfrak{M}, \phi(A) \equiv k^{*} \eta(A) k$ defines a representation of $\mathfrak{C}$ on $\mathcal{K}$. By the von Neumann density theorem $\eta(\mathfrak{C})$ is $\sigma$ strongly dense in $\mathfrak{M}$ and one has $\overline{\phi(\mathfrak{C}) \Phi}=\overline{k^{*} \eta(\mathfrak{C}) \Phi}=\overline{k^{*} \mathfrak{M} \Phi}=\mathcal{K}$. Finally $\mu(A)=(\Phi, \eta(A) \Phi)=(\Phi, \phi(A) \Phi)$. Thus $(\mathcal{K}, \phi, \Phi)$ is the required GNS representation. Since $\mathrm{e}^{i t L} \eta(A) \Phi=\eta\left(\tau^{t}(A)\right) \Phi$ the subspace $\mathcal{K}$ reduces $L$ and the restriction of $L$ to $\mathcal{K}$ is the $\mu$-Liouvillean.

If $\left(\mathfrak{C}, \tau^{t}, \omega\right)$ is a quantum dynamical system and $\mu$ is a $\tau^{t}$-invariant $\omega$ normal state on $\mathfrak{C}$ then $\left(\mathfrak{C}, \tau^{t}, \mu\right)$ is also a quantum dynamical system. The standard Liouvilleans of these two systems are not independent. Their relation is explicited in the next Theorem.

Theorem 15. Let $\left(\mathfrak{C}, \tau^{t}, \omega\right)$ be a quantum dynamical system with standard form $(\eta, \mathfrak{M}, \mathcal{H}, J, \mathcal{C}, L, \Phi)$ and $\mu$ a $\tau^{t}$-invariant $\omega$-normal state. Denote by $P \in$ $\mathfrak{Z}(\mathfrak{M})$ the central support of the normal extension of $\mu$ to $\mathfrak{M}$. The standard Liouvillean of the quantum dynamical system $\left(\mathfrak{C}, \tau^{t}, \mu\right)$ is (unitarily equivalent to) the restriction of $L$ to the subspace PH. In particular, its spectrum is contained in $\sigma(L)$.

Proof. We construct a standard form $\left(\eta_{\mu}, \mathfrak{M}_{\mu}, \mathcal{H}_{\mu}, J_{\mu}, \mathcal{C}_{\mu}, L_{\mu}, \Phi_{\mu}\right)$ of $\left(\mathfrak{C}, \tau^{t}, \mu\right)$. Since $\mu$ is $\omega$-normal there exists $\tilde{\mu} \in N\left(\mathfrak{C}_{\omega}\right)$ with central support $z_{\mu \mid \omega}$ such that $\mu=\tilde{\mu} \circ \pi_{\omega}$. There is also a $*$-isomorphism $\pi: \mathfrak{C}_{\omega} \rightarrow \mathfrak{M}$ such that $\pi \circ \pi_{\omega}=\eta$. The normal extension of $\mu$ to $\mathfrak{M}$ is $\bar{\mu} \equiv \tilde{\mu} \circ \pi^{-1}$ so that $\mu=\bar{\mu} \circ \eta$. We denote its standard vector representative by $\Phi_{\mu}$. It follows from Lemma 
6 that $P=\left[\mathfrak{M} \vee \mathfrak{M}^{\prime} \Phi_{\mu}\right]=\pi\left(z_{\mu \mid \omega}\right)$ and in particular that $\Phi_{\mu} \in \mathcal{H}_{\mu} \equiv P \mathcal{H}$. By Remark 2 one has $\mathfrak{M}=P \mathfrak{M} \oplus(I-P) \mathfrak{M}$ and we can identify $\mathfrak{M}_{\mu} \equiv P \mathfrak{M}$ with a von Neumann algebra on $\mathcal{H}_{\mu}$. Clearly $\eta_{\mu}(A) \equiv P \eta(A)$ defines a representation of $\mathfrak{C}$ in $\mathcal{H}_{\mu}$. By Property (iv) of Definition 18 one has $J P J=P$ so that the subspace $\mathcal{H}_{\mu}$ reduces $J$. It follows that the restriction $J_{\mu}$ of $J$ to this subspace is an anti-unitary involution. Finally, $\mathcal{C}_{\mu} \equiv P \mathcal{C}$ is a cone in $\mathcal{H}_{\mu}$ and

$$
\begin{aligned}
\hat{\mathcal{C}}_{\mu} & =\left\{\psi \in \mathcal{H}_{\mu} \mid(\psi, P \phi) \geq 0 \text { for all } \phi \in \mathcal{C}\right\} \\
& =\left\{\psi \in \mathcal{H}_{\mu} \mid(P \psi, \phi) \geq 0 \text { for all } \phi \in \mathcal{C}\right\} \\
& =\left\{\psi \in \mathcal{H}_{\mu} \mid(\psi, \phi) \geq 0 \text { for all } \phi \in \mathcal{C}\right\} \\
& =\mathcal{H}_{\mu} \cap \mathcal{C} .
\end{aligned}
$$

Let me show that $\mathcal{H}_{\mu} \cap \mathcal{C}=\mathcal{C}_{\mu}$. On the one hand $P=J P J$ yields $P=P^{2}=$ $P J P J$ and hence $P \mathcal{C}=P J P J \mathcal{C}=P J P \mathcal{C} \subset \mathcal{C}$ by Properties (ii) and (iii) of Definition 18. But since $P \mathcal{C} \subset \mathcal{H}_{\mu}$ one concludes that $P \mathcal{C} \subset \mathcal{H}_{\mu} \cap \mathcal{C}$. On the other hand if $\psi \in \mathcal{H}_{\mu} \cap \mathcal{C}$ then $\psi=P \psi \in \mathcal{C}$ and hence $\psi \in P \mathcal{C}$ proving the claim and hence the fact that $\mathcal{C}_{\mu}$ is self-dual.

We have to show that Properties (i)-(iv) of Definition 18 are satisfied. By Remark 2, $\mathfrak{M}_{\mu}^{\prime}=P \mathfrak{M}^{\prime}$ and Property (i) follows from $J_{\mu} \mathfrak{M}_{\mu} J_{\mu}=J P \mathfrak{M} P J=$ $P J \mathfrak{M} J P=P \mathfrak{M}^{\prime} P=\mathfrak{M}_{\mu}^{\prime}$. To prove Property (ii) note that for $\psi \in \mathcal{C}_{\mu} \subset \mathcal{C}$ we have $J_{\mu} \psi=J \psi=\psi$. For $A \in \mathfrak{M}_{\mu}$ we further have $A=A P=P A=P A P$ and hence $A J_{\mu} A \mathcal{C}_{\mu}=P(A P J A P \mathcal{C}) \subset P \mathcal{C}=\mathcal{C}_{\mu}$ which proves Property (iii). By Remark $2, \mathfrak{Z}\left(\mathfrak{M}_{\mu}\right)=P \mathfrak{Z}(\mathfrak{M})$ so that if $A \in \mathfrak{Z}\left(\mathfrak{M}_{\mu}\right)$ we have $J_{\mu} A J_{\mu}=$ $J P A P J=(P A P)^{*}=P A^{*} P=A^{*}$ and Property (iv) is verified.

By Theorem 4, $\hat{\pi}_{\mu \mid \omega}: z_{\mu \mid \omega} \mathfrak{C}_{\omega} \rightarrow \mathfrak{C}_{\mu}$ is a $*$-isomorphism. Hence $\phi \equiv \pi \circ \hat{\pi}_{\mu \mid \omega}^{-1}$ is a faithful representation of $\mathfrak{C}_{\mu}$ in $\mathcal{H}_{\mu}$ such that $\phi\left(\mathfrak{C}_{\mu}\right)=\pi\left(z_{\mu \mid \omega} \mathfrak{C}_{\omega}\right)=P \mathfrak{M}=$ $\mathfrak{M}_{\mu}$ and $\left(\mathfrak{M}_{\mu}, \mathcal{H}_{\mu}, J_{\mu}, \mathcal{C}_{\mu}\right)$ is indeed a standard form of $\mathfrak{C}_{\mu}$. To determine the standard Liouvillean note that since $\bar{\mu}$ is an invariant state its central support $P$ is also invariant

$$
\mathrm{e}^{i t L} P \mathrm{e}^{-i t L}=P
$$

It follows that $P$ reduces the standard Liouvillean $L$. By Equ. (26), we have $\hat{\pi}_{\mu \mid \omega}^{-1} \circ \hat{\tau}_{\mu}^{t}=\hat{\tau}_{\omega}^{t} \circ \hat{\pi}_{\mu \mid \omega}^{-1}$ from which we derive

$$
\phi \circ \hat{\tau}_{\mu}^{t}(A)=\pi \circ \hat{\tau}_{\omega}^{t} \circ \hat{\pi}_{\mu \mid \omega}^{-1}(A)=\mathrm{e}^{i t L} \phi(A) \mathrm{e}^{-i t L} .
$$

Finally, since $\mathrm{e}^{i t L} \mathcal{C}_{\mu}=\mathrm{e}^{i t L} P \mathcal{C}=P \mathrm{e}^{i t L} \mathcal{C}=P \mathcal{C}=\mathcal{C}_{\mu}$ we conclude that the standard Liouvillean of $\hat{\tau}_{\mu}^{t}$ is the restriction of $L$ to $\mathcal{H}_{\mu}$.

Example 11. (Standard form of a finite quantum system) Consider the quantum dynamical system $\left(\mathfrak{M}, \tau^{t}, \mu\right)$ of Example 9 and suppose that the Hilbert space $\mathcal{H}$ is separable. To construct a standard form let $\rho_{0}$ be a density matrix on $\mathcal{H}$ such that $\operatorname{Ker} \rho_{0}=\{0\}$. It follows that $\overline{\operatorname{Ran} \rho_{0}}=\mathcal{H}$ and Lemma 3 yields that the normal state $\omega_{0}(A) \equiv \operatorname{Tr}\left(\rho_{0} A\right)$ is faithful. The GNS representation of $\mathfrak{M}$ corresponding to $\omega_{0}$ is given, according to Example 9 , by 


$$
\begin{aligned}
\mathcal{H}_{\omega_{0}} & =\mathcal{L}^{2}(\mathcal{H}), \\
\pi_{\omega_{0}}(A) X & =A X, \\
\Omega_{\omega_{0}} & =\rho_{0}^{1 / 2} .
\end{aligned}
$$

By Lemma 6 this representation is faithful and by Lemma 9 the corresponding enveloping von Neumann algebra is $\mathfrak{M}_{\omega_{0}}=\pi_{\omega_{0}}(\mathfrak{M})$. It follows that $\hat{\omega}_{0}$ is faithful on $\mathfrak{M}_{\omega_{0}}$ and hence, by Proposition 5 , that $\mathfrak{M}_{\omega_{0}}$ is in standard form. Indeed, one easily checks that $J: X \mapsto X^{*}$ and $\mathcal{C} \equiv\left\{X \in \mathcal{L}^{2}(\mathcal{H}) \mid X \geq 0\right\}$ satisfy all the conditions of Definition 18.

By Proposition 4, the standard Liouvillean $L$ is given by

$$
\mathrm{e}^{i t L} X=\mathrm{e}^{i t H} X \mathrm{e}^{-i t H},
$$

since this is a unitary implementation of the dynamics

$$
\mathrm{e}^{i t L} \pi_{\omega_{0}}(A) \mathrm{e}^{-i t L} X=\mathrm{e}^{i t H}\left(A \mathrm{e}^{-i t H} X \mathrm{e}^{i t H}\right) \mathrm{e}^{-i t H}=\pi_{\omega_{0}}\left(\tau^{t}(A)\right) X,
$$

which preserves the cone $\mathcal{C}$. The standard vector representative of the invariant state $\mu(A)=\operatorname{Tr}(\rho A)$ is $\rho^{1 / 2} \in \mathcal{C}$. One easily checks that the map $\left.X \mapsto X\right|_{\mathcal{G}}$ is isometric from $\mathfrak{M}_{\omega_{0}} \rho^{1 / 2}$ into $\mathcal{L}^{2}(\mathcal{G}, \mathcal{H})$ and has a dense range. It extends to a unitary map $U$ between $\mathcal{K} \equiv \overline{\mathfrak{M}_{\omega_{0}} \rho^{1 / 2}}$ and $\mathcal{H}_{\mu}=\mathcal{L}^{2}(\mathcal{G}, \mathcal{H})$ such that, according to Proposition 6

$$
\left.U L\right|_{\mathcal{K}} U^{*}=L_{\mu}
$$

\subsection{Ergodic Properties of Quantum Dynamical Systems}

My aim in this subsection is to extend to quantum dynamics the definitions and characterizations of the ergodic properties introduced in Subsection 3.1. Since there is no obvious way to formulate a quantum individual ergodic theorem (Theorem 5), I shall use the characterization (iv) of Proposition 1 to define ergodicity. Mixing will then be defined using Definition 6. To do so I only need to extend the notion of absolute continuity of two measures to two states on a $C^{*}$-algebra.

Example 12. Consider the classical $C^{*}$-dynamical system $\left(C(M), \tau^{t}\right)$ of Example 4 and let $\mu$ be a $\tau^{t}$-invariant Baire probability measure on $M$. To construct the associated GNS representation denote by $\hat{\mu}$ the regular Borel extension of $\mu$ and set $\mathcal{H}_{\mu} \equiv L^{2}(M, \mathrm{~d} \hat{\mu})$. The $C^{*}$-algebra $C(M)$ is represented on this Hilbert space as

$$
\pi_{\mu}(f): \psi \mapsto f \psi .
$$

Since $C(M)$ is dense in $L^{2}(M, \mathrm{~d} \mu)$ the vector $\Omega_{\mu}=1$ is cyclic. Finally, one has

$$
\left(\Omega_{\mu}, \pi_{\mu}(f) \Omega_{\mu}\right)=\int f(x) \mathrm{d} \hat{\mu}(x)=\mu(f) .
$$


We note in passing that the corresponding enveloping von Neumann algebra is $\pi_{\mu}(C(M))^{\prime \prime}=L^{\infty}(M, \mathrm{~d} \hat{\mu})$.

If $\nu$ is a $\mu$-normal state on $C(M)$ then there exists a density matrix $\rho$ on $\mathcal{H}_{\mu}$ such that $\nu(f)=\operatorname{Tr}\left(\rho \pi_{\mu}(f)\right)$. Let

$$
\rho=\sum_{n} p_{n} \psi_{n}\left(\psi_{n}, \cdot\right)
$$

be the spectral representation of $\rho$. It follows that

$$
F(x) \equiv \sum_{n} p_{n}\left|\psi_{n}(x)\right|^{2} \in L^{1}(M, \mathrm{~d} \hat{\mu}),
$$

and hence

$$
\nu(f)=\int F(x) f(x) \mathrm{d} \hat{\mu}(x) .
$$

Thus, if $\nu$ is $\mu$-normal its regular Borel extension $\hat{\nu}$ is absolutely continuous with respect to $\hat{\mu}$.

Reciprocally, if $\hat{\nu} \ll \hat{\mu}$ and $G=\left(\frac{\mathrm{d} \hat{\nu}}{\mathrm{d} \hat{\mu}}\right)^{1 / 2}$ one has

$$
\nu(f)=\int f \frac{\mathrm{d} \hat{\nu}}{\mathrm{d} \hat{\mu}} \mathrm{d} \hat{\mu}=\left(G, \pi_{\mu}(f) G\right),
$$

and we conclude that $\nu$ is $\mu$-normal.

This example shows that for Abelian $C^{*}$ - or $W^{*}$-algebras absolute continuity is equivalent to relative normality. If $A \subset M$ is a measurable set then its characteristic function $\chi_{A}$, viewed as a multiplication operator on $\mathcal{H}_{\mu}$, is an orthogonal projection. In fact all orthogonal projections in $\pi_{\mu}(C(M))^{\prime \prime}$ are easily seen to be of this form. Since the absolute continuity of the Borel measure $\hat{\nu}$ with respect to $\hat{\mu}$ means that $\hat{\mu}\left(\chi_{A}\right)=0$ implies $\hat{\nu}\left(\chi_{A}\right)=0$ we see that if $\nu$ is $\mu$-normal then $s_{\nu} \leq s_{\mu}$. As the following example shows this is not necessarily true in the non-Abelian case.

Example 13. Let $\mathfrak{M} \equiv \mathfrak{B}(\mathcal{H})$ with $\operatorname{dim} \mathcal{H}>1$ and $\mu \equiv(\psi, \cdot \psi)$ for some unit vector $\psi \in \mathcal{H}$. As already remarked in Example 9 , any normal state on $\mathfrak{M}$ is $\mu$-normal. In particular if $\rho$ is a density matrix such that Ker $\rho=\{0\}$ then $\nu \equiv \operatorname{Tr}(\rho \cdot)$ is a faithful $\mu$-normal state and hence $I=s_{\nu}>s_{\mu}=\psi(\psi, \cdot)$.

Considering the special case of a $W^{*}$-dynamical system $\left(\mathfrak{M}, \tau^{t}\right)$ equipped with a normal invariant state $\mu$, the following argument shows that the condition $s_{\nu} \leq s_{\mu}$ is necessary if we wish to define the ergodicity of the quantum dynamical system $\left(\mathfrak{M}, \tau^{t}, \mu\right)$ by the condition (iv) of Proposition 1 , i.e.,

$$
\lim _{T \rightarrow \infty} \frac{1}{T} \int_{0}^{T} \nu \circ \tau^{t}(A) \mathrm{d} t=\mu(A),
$$

for all $A \in \mathfrak{M}$. Indeed, since $\mu$ is $\tau^{t}$-invariant we have $\tau^{t}\left(s_{\mu}\right)=s_{\mu}$ and Equ. (32) leads to 


$$
\nu\left(s_{\mu}\right)=\lim _{T \rightarrow \infty} \frac{1}{T} \int_{0}^{T} \nu \circ \tau^{t}\left(s_{\mu}\right) \mathrm{d} t=\mu\left(s_{\mu}\right)=1,
$$

which means that $s_{\nu} \leq s_{\mu}$.

These considerations motivate the following definition.

Definition 23. If $\nu, \mu \in E(\mathfrak{C})$ are such that $\nu \in N(\mathfrak{C}, \mu)$ and $s_{\nu} \leq s_{\mu}$ then we say that $\nu$ is absolutely continuous with respect to $\mu$ and we write $\nu \ll \mu$. We also set

$$
\mathcal{S}(\mathfrak{C}, \mu) \equiv\{\nu \in E(\mathfrak{C}) \mid \nu \ll \mu\} .
$$

When dealing with the set $\mathcal{S}(\mathfrak{C}, \mu)$, the following lemma is often useful.

Lemma 13. Let $\mathfrak{C}$ be a $C^{*}$-algebra, $\mu \in E(\mathfrak{C})$ and for $\lambda>0$ set

$$
\mathcal{S}^{\lambda}(\mathfrak{C}, \mu) \equiv\{\nu \in E(\mathfrak{C}) \mid \nu \leq \lambda \mu\}
$$

(i) $\mathcal{S}^{\lambda}(\mathfrak{C}, \mu)$ is weak-^ compact.

(ii) The set

$$
S_{0}(\mathfrak{C}, \mu) \equiv \bigcup_{n=1}^{\infty} \mathcal{S}^{n}(\mathfrak{C}, \mu)
$$

is total in $\mathcal{S}(\mathfrak{C}, \mu)$, i.e., the set of finite convex linear combinations of elements of $\mathcal{S}_{0}(\mathfrak{C}, \mu)$ is a norm dense subset of $\mathcal{S}(\mathfrak{C}, \mu)$.

(iii) If $(\pi, \mathcal{H})$ is a representation of $\mathfrak{C}$ such that $\mu(A)=(\Psi, \pi(A) \Psi)$ for some $\Psi \in \mathcal{H}$ then one has

$$
\mathcal{S}_{0}(\mathfrak{C}, \mu)=\left\{(\Phi, \pi(\cdot) \Phi) \mid \Phi \in \pi(\mathfrak{C})^{\prime} \Psi,\|\Phi\|=1\right\} .
$$

Proof. We first prove (iii). Assume that $\nu \in \mathcal{S}_{0}(\mathfrak{C}, \mu)$ as defined in Equ. (33). Then there exists $\lambda>0$ such that $\nu\left(A^{*} A\right) \leq \lambda \mu\left(A^{*} A\right)=\lambda\|\pi(A) \Psi\|^{2}$ for all $A \in \mathfrak{C}$. By the Cauchy-Schwarz inequality we further get

$$
\left|\nu\left(A^{*} B\right)\right| \leq \lambda\|\pi(A) \Psi\|\|\pi(B) \Psi\|
$$

for all $A, B \in \mathfrak{C}$. It follows that the map

$$
\begin{aligned}
\pi(\mathfrak{C}) \Psi \times \pi(\mathfrak{C}) \Psi & \rightarrow \mathbb{C} \\
(\pi(A) \Psi, \pi(B) \Psi) & \mapsto \nu\left(A^{*} B\right),
\end{aligned}
$$

is well defined. As a densely defined, bounded, non-negative sesquilinear form on $\mathcal{K} \equiv \overline{\pi(\mathfrak{C}) \Psi}$ it defines a bounded non-negative self-adjoint operator $M \in \mathfrak{B}(\mathcal{K})$, which we can extend by 0 on $\mathcal{K}^{\perp}$ and such that $\nu\left(A^{*} B\right)=$ $(\pi(A) \Psi, M \pi(B) \Psi)$. Since $\nu\left(A^{*} B C\right)=\nu\left(\left(B^{*} A\right)^{*} C\right)$ we get

$$
(\pi(A) \Psi, M \pi(B) \pi(C) \Psi)=(\pi(A) \Psi, \pi(B) M \pi(C) \Psi),
$$

for all $A, B, C \in \mathfrak{C}$. Since $M$ vanishes on $\mathcal{K}^{\perp}$ we can conclude that $M \in \pi(\mathfrak{C})^{\prime}$. Set $R \equiv M^{1 / 2}$, it follows that $R \in \pi(\mathfrak{C})^{\prime}$, 


$$
\nu(A)=(R \Psi, \pi(A) R \Psi),
$$

and $\|R \Psi\|=1$. Reciprocally, if $\nu$ is given by the above formula one has $\nu\left(A^{*} A\right)=\|R \pi(A) \Psi\| \leq\|R\|\|\pi(A) \Psi\|^{2}=\|R\| \mu\left(A^{*} A\right)$ and thus $\nu \in S_{0}(\mathfrak{C}, \mu)$.

To prove (ii) let $\left(\mathcal{H}_{\mu}, \pi_{\mu}, \Omega_{\mu}\right)$ be the GNS representation of $\mathfrak{C}$ associated to $\mu$. If $\nu \in \mathcal{S}(\mathfrak{C}, \mu)$ then there exists $\tilde{\nu} \in N\left(\mathfrak{C}_{\mu}\right)$ such that $\nu=\tilde{\nu} \circ \pi_{\mu}$ and $\tilde{\nu}\left(s_{\mu}\right)=1$. Let $\rho$ be a density matrix on $\mathcal{H}_{\mu}$ such that $\tilde{\nu}(A)=\operatorname{Tr}(\rho A)$. From the condition $\operatorname{Tr}\left(\rho s_{\mu}\right)=1$ it follows that $\rho=s_{\mu} \rho s_{\mu}$. Thus, $\rho$ is a density matrix in the subspace Ran $s_{\mu}=\overline{\mathfrak{C}_{\mu}^{\prime} \Omega_{\mu}}$ and Exercise 3 shows that $\tilde{\nu}$ can be approximated in norm by finite convex linear combinations of vector states $\tilde{\nu}_{n}(A)=\left(\psi_{n}, A \psi_{n}\right)$ with $\psi_{n} \in \mathfrak{C}_{\mu}^{\prime} \Omega_{\mu}$. (ii) now follows from (iii) and the fact that the map $\pi_{\mu}^{\star}: \tilde{\nu} \mapsto \tilde{\nu} \circ \pi_{\mu}$ is norm continuous.

Since $E(\mathfrak{C})$ is weak- $\star$ compact in $\mathfrak{C}^{\star}$ (i) follows from the obvious fact that $\mathcal{S}^{\lambda}(\mathfrak{C}, \mu)$ is weak- $\star$ closed.

Definition 24. A quantum dynamical system $\left(\mathfrak{C}, \tau^{t}, \mu\right)$ is called:

(i) Ergodic if, for any $\nu \ll \mu$ and any $A \in \mathfrak{C}$, one has

$$
\lim _{T \rightarrow \infty} \frac{1}{T} \int_{0}^{T} \nu \circ \tau^{t}(A) \mathrm{d} t=\mu(A) .
$$

(ii) Mixing if, for any $\nu \ll \mu$ and any $A \in \mathfrak{C}$, one has

$$
\lim _{t \rightarrow \infty} \nu \circ \tau^{t}(A)=\mu(A) .
$$

A state $\mu \in E(\mathfrak{C})$ is called ergodic (resp. mixing) if it belongs to $\mathcal{E}\left(\mathfrak{C}, \tau^{t}\right)$ and if the corresponding quantum dynamical system $\left(\mathfrak{C}, \tau^{t}, \mu\right)$ is ergodic (resp. mixing).

These definitions are consistent with the notion of normal form of a quantum dynamical system.

Proposition 7. The quantum dynamical system $\left(\mathfrak{C}, \tau^{t}, \mu\right)$ is ergodic (resp. mixing) if and only if its normal form $\left(\pi_{\mu}, \mathfrak{C}_{\mu}, \mathcal{H}_{\mu}, L_{\mu}, \Omega_{\mu}\right)$ is ergodic (resp. mixing).

Proof. We denote by $\hat{\mu}$ the vector state associated to $\Omega_{\mu}$ and $\hat{\tau}_{\mu}^{t}$ the $\sigma$-weakly continuous group of $*$-automorphisms of $\mathfrak{C}_{\mu}$ generated by $L_{\mu}$. Assume that $\left(\mathfrak{C}_{\mu}, \hat{\tau}_{\mu}^{t}, \hat{\mu}\right)$ is ergodic (resp. mixing) and let $\nu \in \mathcal{S}(\mathfrak{C}, \mu)$ and $A \in \mathfrak{C}$. Then there exists $\tilde{\nu} \in N\left(\mathfrak{C}_{\mu}\right)$ such that $\tilde{\nu} \ll \hat{\mu}$ and $\nu=\tilde{\nu} \circ \pi_{\mu}$. Since $\nu \circ \tau^{t}(A)=$ $\tilde{\nu} \circ \hat{\tau}_{\mu}^{t}\left(\pi_{\mu}(A)\right)$ and $\mu(A)=\hat{\mu}\left(\pi_{\mu}(A)\right)$ the convergence in Equ. (34) (resp. Equ. (35)) follows directly from the corresponding statement for $\left(\mathfrak{C}_{\mu}, \hat{\tau}_{\mu}^{t}, \hat{\mu}\right)$.

To prove the reverse statement assume that $\left(\mathfrak{C}, \tau^{t}, \mu\right)$ is ergodic (resp. mixing). Since $\hat{\tau}_{\mu}^{t}$ is isometric the map $\nu \mapsto \nu \circ \hat{\tau}_{\mu}^{t}$ is norm continuous uniformly in $t \in \mathbb{R}$. To prove convergence in Equ. (34) (resp. Equ. (35)) for all $\nu \in$ $\mathcal{S}\left(\mathfrak{C}_{\mu}, \hat{\mu}\right)$ it is therefore sufficient to prove it for all $\nu$ in a total subset of 
$\mathcal{S}\left(\mathfrak{C}_{\mu}, \hat{\mu}\right)$. By Lemma $13, \mathcal{S}_{0}\left(\mathfrak{C}_{\mu}, \hat{\mu}\right)$ is total in $\mathcal{S}\left(\mathfrak{C}_{\mu}, \hat{\mu}\right)$ and is the union of the sets $\mathcal{S}^{n}\left(\mathfrak{C}_{\mu}, \hat{\mu}\right)$. Thus, it suffices to consider $\nu \in \mathcal{S}^{n}\left(\mathfrak{C}_{\mu}, \hat{\mu}\right)$ for some $n>0$. We set

$$
\nu_{t}(A) \equiv \frac{1}{t} \int_{0}^{t} \nu \circ \hat{\tau}_{\mu}^{s}(A) \mathrm{d} s,\left(\text { resp. } \nu_{t}(A) \equiv \nu \circ \hat{\tau}_{\mu}^{t}(A)\right) .
$$

It follows from the fact that $\hat{\mu}$ is $\hat{\tau}_{\mu}^{t}$-invariant that $\nu_{t} \in S^{n}\left(\mathfrak{C}_{\mu}, \hat{\mu}\right)$ for all $t \in \mathbb{R}$.

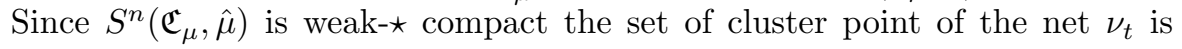
non-empty and contained in $S^{n}\left(\mathfrak{C}_{\mu}, \hat{\mu}\right) \subset N\left(\mathfrak{C}_{\mu}\right)$. If $\bar{\nu}$ is such a cluster point then it follows from $\nu \circ \hat{\tau}_{\mu}^{t}\left(\pi_{\mu}(A)\right)=\left(\nu \circ \pi_{\mu}\right) \circ \tau^{t}(A)$ and $\nu \circ \pi_{\mu} \ll \mu$ that $\bar{\nu}\left(\pi_{\mu}(A)\right)=\hat{\mu}\left(\pi_{\mu}(A)\right)$. Since $\bar{\nu}$ is normal we conclude that $\bar{\nu}=\hat{\mu}$ and hence that $\hat{\mu}$ is the weak- $\star$ limit of the net $\nu_{t}$.

Example 14. (Finite quantum systems, continuation of Example 9) If $\psi$ is a normalized eigenvector of the Hamiltonian $H$ then the state $\mu \equiv(\psi,(\cdot) \psi)$ is ergodic and even mixing. Indeed, since $s_{\mu}=\psi(\psi, \cdot)$, the only state $\nu$ satisfying $\nu \ll \mu$ is $\mu$ itself. Any normal invariant state which is a mixture of several such eigenstates is non-ergodic. In fact, the formula

$$
\rho(A)=\sum_{j} p_{j}\left(\psi_{j}, A \psi_{j}\right)
$$

expresses the decomposition of the state $\rho$ into ergodic components.

Remark 7. Proposition 7 reduces the study of the ergodic properties of a quantum dynamical system to the special case of a $W^{*}$-dynamical systems $\left(\mathfrak{M}, \tau^{t}, \mu\right)$ with $\mu \in N\left(\mathfrak{M}, \tau^{t}\right)$. For such a quantum dynamical system one has

$$
N(\mathfrak{M}, \mu)=\left\{\nu \in N(\mathfrak{M}) \mid s_{\nu} \leq z_{\mu}\right\},
$$

by Lemma 10. Since $s_{\mu} \leq z_{\mu}$ we conclude that $\nu \ll \mu$ if and only if $\nu \in N(\mathfrak{M})$ and $\nu\left(s_{\mu}\right)=1$, i.e., $\nu=\nu\left(s_{\mu}(\cdot) s_{\mu}\right)$. For $\nu \in N(\mathfrak{M})$ one has either $\nu\left(s_{\mu}\right)=0$ and thus $\nu\left(s_{\mu}(\cdot) s_{\mu}\right)=0$ or $\nu\left(s_{\mu}(\cdot) s_{\mu}\right) / \nu\left(s_{\mu}\right) \ll \mu$. We conclude that

$$
\{\nu \in E(\mathfrak{M}) \mid \nu \ll \mu\}=\left\{\nu\left(s_{\mu}(\cdot) s_{\mu}\right) \mid \nu \in \mathfrak{M}_{\star}, \nu \geq 0, \nu\left(s_{\mu}\right)=1\right\} .
$$

In particular, the set of linear combinations of states $\nu \ll \mu$ is the set of linear functionals of the form $\nu\left(s_{\mu}(\cdot) s_{\mu}\right)$ where $\nu \in \mathfrak{M}_{\star}$.

As a quantum analogue of Proposition 1 we have the following characterizations of ergodicity which, using Proposition 7, can be applied to the normal form of a quantum dynamical system.

Proposition 8. Let $\left(\mathfrak{M}, \tau^{t}\right)$ be a $W^{*}$-dynamical system and $\mu \in N\left(\mathfrak{M}, \tau^{t}\right)$. Denote by $\mathfrak{M}_{\tau, \mu} \equiv\left\{A \in s_{\mu} \mathfrak{M} s_{\mu} \mid \tau^{t}(A)=A\right.$ for all $\left.t \in \mathbb{R}\right\}$ the subalgebra of $\tau^{t}$-invariant observables modulo $\mu$. The following propositions are equivalent.

(i) $\left(\mathfrak{M}, \tau^{t}, \mu\right)$ is ergodic.

(ii) If $P \in \mathfrak{M}_{\tau, \mu}$ is an orthogonal projection then $\mu(P) \in\{0,1\}$. 
(iii) If $A \in \mathfrak{M}_{\tau, \mu}$ then $A=\mu(A) s_{\mu}$.

Proof. (i) $\Rightarrow$ (ii). By Remark 7, the system is ergodic if and only if

$$
\lim _{T \rightarrow \infty} \frac{1}{T} \int_{0}^{T} \nu \circ \tau^{t}\left(s_{\mu} A s_{\mu}\right) \mathrm{d} t=\nu\left(s_{\mu}\right) \mu(A),
$$

holds for all $A \in \mathfrak{M}$ and $\nu \in \mathfrak{M}_{\star}$. Let $P \in \mathfrak{M}_{\tau, \mu}$ be an orthogonal projection. Since $P=s_{\mu} P s_{\mu}$ is $\tau^{t}$-invariant Equ. (37) yields $\nu(P)=\nu\left(s_{\mu}\right) \mu(P)$, i.e., $\nu\left(P-\mu(P) s_{\mu}\right)=0$ for any $\nu \in \mathfrak{M}_{\star}$. Thus, $P=\mu(P) s_{\mu}$ and from

$$
0=P(I-P)=\mu(P) s_{\mu}\left(I-\mu(P) s_{\mu}\right)=\mu(P)(1-\mu(P)) s_{\mu},
$$

we conclude that $\mu(P) \in\{0,1\}$.

(ii) $\Rightarrow$ (iii). It clearly suffices to prove that $A=\mu(A) s_{\mu}$ for all self-adjoint elements of $\mathfrak{M}_{\tau, \mu}$. Let $A \in \mathfrak{M}_{\tau, \mu}$ be self-adjoint and denote by $P_{a}$ its spectral projection corresponding to the interval $]-\infty, a]$. Note that if $\mathfrak{M} \subset \mathfrak{B}(\mathcal{H})$ then we can interpret $\mathfrak{M}_{\tau, \mu}$ as a von Neumann algebra on $s_{\mu} \mathcal{H}$ and the functional calculus yields $P_{a} \in \mathfrak{M}_{\tau, \mu}$. It follows from (ii) that for any $a \in \mathbb{R}$ either $\mu\left(P_{a}\right)=0$ or $\mu\left(P_{a}\right)=1$. In the first case we get $P_{a} s_{\mu}=0$ and hence $P_{a}=0$. In the second case we have $P_{a} \geq s_{\mu}$ and hence $P_{a}=s_{\mu}$. By the spectral theorem $A$ is a multiple of $s_{\mu}$ and (iii) follows.

(iii) $\Rightarrow($ i). Let $\nu \ll \mu$ and $\tilde{\nu} \in E(\mathfrak{M})$ be a weak- $\star$ cluster point of the net

$$
\nu_{T} \equiv \frac{1}{T} \int_{0}^{T} \nu \circ \tau^{t} \mathrm{~d} t
$$

Denote by $T_{\alpha}$ a net such that $\lim _{\alpha} \nu_{T_{\alpha}}(A)=\tilde{\nu}(A)$ for all $A \in \mathfrak{M}$. For $A \in \mathfrak{M}$ consider the corresponding net

$$
A_{T_{\alpha}} \equiv \frac{1}{T_{\alpha}} \int_{0}^{T_{\alpha}} \tau^{t}(A) \mathrm{d} t \in \mathfrak{M}_{\|A\|} \equiv\{B \in \mathfrak{M} \mid\|B\| \leq\|A\|\} .
$$

By the Banach-Alaoglu theorem $\mathfrak{M}_{\|A\|}$ is $\sigma$-weakly compact and there exists a subnet $T_{\beta}$ of the net $T_{\alpha}$ such that $A_{T_{\beta}} \rightarrow \tilde{A} \sigma$-weakly. Since $\mu$ is $\sigma$-weakly continuous and $\tau^{t}$-invariant $\mu(\tilde{A})=\mu(A)$. By the usual argument (see the proof of Theorem 10) we have $\tau^{t}(\tilde{A})=\tilde{A}$ for all $t$. Since $\mu$ is $\tau^{t}$-invariant we also have $\tau^{t}\left(s_{\mu}\right)=s_{\mu}$ and hence $s_{\mu} \tilde{A} s_{\mu} \in \mathfrak{M}_{\tau, \mu}$. It follows from (iii) that $s_{\mu} \tilde{A} s_{\mu}=\mu\left(s_{\mu} \tilde{A} s_{\mu}\right) s_{\mu}=\mu(\tilde{A}) s_{\mu}=\mu(A) s_{\mu}$. On the other hand, since $\nu$ is $\sigma$-weakly continuous and $\nu\left(s_{\mu}\right)=1$, we have

$$
\tilde{\nu}(A)=\lim _{\alpha} \nu_{T_{\alpha}}(A)=\lim _{\beta} \nu_{T_{\beta}}(A)=\lim _{\beta} \nu\left(A_{T_{\beta}}\right)=\nu(\tilde{A})=\nu\left(s_{\mu} \tilde{A} s_{\mu}\right),
$$

and hence $\tilde{\nu}(A)=\nu\left(\mu(A) s_{\mu}\right)=\mu(A)$. Since this holds for any $A \in \mathfrak{M}$ and any cluster point of the net $\nu_{T}$ we conclude that $\nu_{T}$ converges to $\mu$ in the weak- $\star$ topology, i.e., that Equ. (34) holds. 
Note that if $\left(\mathfrak{M}, \tau^{t}, \mu\right)$ is ergodic and $\mu$ is faithful $\left(s_{\mu}=I\right)$ it follows immediately that $\mu$ is the only normal invariant state of the $W^{*}$-dynamical system $\left(\mathfrak{M}, \tau^{t}\right)$. More generally, one has

Proposition 9. If the quantum dynamical systems $\left(\mathfrak{C}, \tau^{t}, \mu_{1}\right),\left(\mathfrak{C}, \tau^{t}, \mu_{2}\right)$ are ergodic then either $\mu_{1}=\mu_{2}$ or $\mu_{1} \perp \mu_{2}$.

Proof. Since $\mu_{1}, \mu_{2} \in \mathcal{E}\left(\mathfrak{C}, \tau^{t}\right)$ we have $\omega \equiv\left(\mu_{1}+\mu_{2}\right) / 2 \in \mathcal{E}\left(\mathfrak{C}, \tau^{t}\right)$ and hence $\left(\mathfrak{C}, \tau^{t}, \omega\right)$ is a quantum dynamical system. Let $\left(\mathfrak{C}_{\omega}, \hat{\tau}_{\omega}^{t}, \hat{\omega}\right)$ be its normal form. From the fact that $\mu_{i} \leq 2 \omega$ we conclude by Lemma 13 that $\mu_{1}$ and $\mu_{2}$ are $\omega$-normal. Denote by $\tilde{\mu}_{i}$ the normal extension of $\mu_{i}$ to $\mathfrak{C}_{\omega}, s_{i} \equiv s_{\mu_{i} \mid \omega}$ its support, $z_{i} \equiv z_{\mu_{i} \mid \omega}$ its central support and set $\mathfrak{M}_{i} \equiv z_{i} \mathfrak{C}_{\omega}$. By Remark 6 the quantum dynamical system $\left(\mathfrak{M}_{i}, \hat{\tau}_{\omega}^{t}, \tilde{\mu}_{i}\right)$ is isomorphic to the normal form $\left(\mathfrak{C}_{\mu_{i}}, \hat{\tau}_{\mu_{i}}^{t}, \hat{\mu}_{i}\right)$ of $\left(\mathfrak{C}, \tau^{t}, \mu_{i}\right)$ and is therefore ergodic. Since the supports $s_{1}, s_{2}$ are invariant under $\hat{\tau}_{\omega}^{t}$ so is $P \equiv s_{1} \wedge s_{2}$. From $s_{i} \leq z_{i}$ we conclude that $P \leq z_{1} \wedge z_{2} \leq z_{i}$ and hence $P \in \mathfrak{M}_{1}^{P} \cap \mathfrak{M}_{2}^{P}$. Since $P=s_{i} P s_{i}$, Proposition 8 yields that $\tilde{\mu}_{i}(P) \in\{0,1\}$. As in the proof of Proposition 8 this implies that $P=\tilde{\mu}_{1}(P) s_{1}=\tilde{\mu}_{2}(P) s_{2}$ and hence either $P=0$ or $P=s_{1}=s_{2}$. In the later case one has $s_{1} \leq s_{2} \leq z_{2}$ and Corollary 2 yields $\mu_{1} \ll \mu_{2}$. It immediately follows from Equ. (34) that $\mu_{1}=\mu_{2}$. In the former case assume that for some $\lambda>0$ and some state $\nu$ one has $\lambda \nu \leq \mu_{i}$ for $i=1,2$. Then $\lambda \nu \leq \omega$ and by Lemma $13, \nu$ has a normal extension $\tilde{\nu}$ to $\mathfrak{C}_{\omega}$ which satisfies $\lambda \tilde{\nu} \leq \tilde{\mu}_{i}$. It follows that $\tilde{\nu}\left(I-s_{1}\right)=\tilde{\nu}\left(I-s_{2}\right)=0$ and hence $\tilde{\nu}\left(s_{1}\right)=\tilde{\nu}\left(s_{2}\right)=1$. By Exercise 6 we get $\tilde{\nu}(P)=1$, a contradiction which shows that $\mu_{1} \perp \mu_{2}$.

Proposition 10. A quantum dynamical system $\left(\mathfrak{C}, \tau^{t}, \mu\right)$ is ergodic if and only if the state $\mu$ is an extremal point of the set $\mathcal{E}\left(\mathfrak{C}, \tau^{t}\right)$.

Proof. Assume that $\left(\mathfrak{C}, \tau^{t}, \mu\right)$ is ergodic and that there exists $\mu_{1}, \mu_{2} \in \mathcal{E}\left(\mathfrak{C}, \tau^{t}\right)$ and $\left.\alpha_{1}, \alpha_{2} \in\right] 0,1\left[\right.$ such that $\alpha_{1}+\alpha_{2}=1$ and $\mu=\alpha_{1} \mu_{1}+\alpha_{2} \mu_{2}$. By Lemma 13 it follows from the fact that $\alpha_{i} \mu_{i} \leq \mu$ that $\mu_{i}$ is $\mu$-normal. Denote by $\tilde{\mu}_{i}$ its normal extension to $\mathfrak{C}_{\mu}$. By continuity one has $\alpha_{i} \tilde{\mu}_{i} \leq \hat{\mu}$ and hence $\tilde{\mu}_{i}\left(I-s_{\mu}\right)=0$, i.e., $s_{\mu_{i}} \leq s_{\mu}$. Equ. (34) then yields $\mu_{i}=\mu$ and we conclude that $\mu$ is extremal.

Assume now that $\mu$ is extremal in $\mathcal{E}\left(\mathfrak{C}, \tau^{t}\right)$ and let $\left(\mathfrak{C}_{\mu}, \hat{\tau}_{\mu}^{t}, \hat{\mu}\right)$ be the corresponding normal form. Let $(\mathcal{H}, \pi)$ be a standard representation of $\mathfrak{C}_{\mu}$ and denote by $(\mathcal{H}, \mathfrak{M}, J, \mathcal{C})$ its standard form. Let $L$ be the corresponding standard Liouvillean and $\Psi_{\mu}$ the standard vector representative of $\hat{\mu}$. The dynamics is implemented in $\mathfrak{M}$ by $\sigma^{t}(A)=\mathrm{e}^{i t L} A \mathrm{e}^{-i t L}$ and if we set $\bar{\mu}(A) \equiv\left(\Psi_{\mu}, A \Psi_{\mu}\right)$ then the normal form is isomorphic to the quantum dynamical system $\left(\mathfrak{M}, \sigma^{t}, \bar{\mu}\right)$.

If $\bar{\nu} \in N\left(\mathfrak{M}, \sigma^{t}\right)$ then $\bar{\nu} \circ \pi \circ \pi_{\mu}$ is $\mu$-normal and invariant, hence it belongs to $\mathcal{E}\left(\mathfrak{C}, \tau^{t}\right)$. In fact, since $\pi_{\mu}(\mathfrak{C})$ is $\sigma$-weakly dense in $\mathfrak{C}_{\mu}$ the map $\bar{\nu} \mapsto \bar{\nu} \circ \pi \circ \pi_{\mu}$ is an injection from $N\left(\mathfrak{M}, \sigma^{t}\right)$ into $\mathcal{E}\left(\mathfrak{C}, \tau^{t}\right)$. It follows that $\bar{\mu}$ is an extremal point of $N\left(\mathfrak{M}, \sigma^{t}\right)$. Indeed, let $\bar{\mu}_{1}, \bar{\mu}_{2} \in N\left(\mathfrak{M}, \sigma^{t}\right)$ and $\left.\alpha \in\right] 0,1[$ be such that $\bar{\mu}=\alpha \bar{\mu}_{1}+(1-\alpha) \bar{\mu}_{2}$. Then we have $\mu=\alpha \bar{\mu}_{1} \circ \pi \circ \pi_{\mu}+(1-\alpha) \bar{\mu}_{2} \circ \pi \circ \pi_{\mu}$ and since $\mu$ is extremal this yields $\bar{\mu}_{1}=\bar{\mu}_{2}$. 
Let $P \in \mathfrak{M}_{\sigma, \bar{\mu}}$ and assume that $\left.\alpha \equiv \bar{\mu}(P) \in\right] 0,1\left[\right.$. Set $P^{\prime} \equiv J P J \in \mathfrak{M}^{\prime}$ and define the states

$$
\bar{\mu}_{1}(A) \equiv \frac{\left(P^{\prime} \Psi_{\mu}, A P^{\prime} \Psi_{\mu}\right)}{\left\|P^{\prime} \Psi_{\mu}\right\|^{2}}, \quad \bar{\mu}_{2}(A) \equiv \frac{\left(\left(I-P^{\prime}\right) \Psi_{\mu}, A\left(I-P^{\prime}\right) \Psi_{\mu}\right)}{\left\|\left(I-P^{\prime}\right) \Psi_{\mu}\right\|^{2}},
$$

on $\mathfrak{M}$. Since $\left\|P^{\prime} \Psi_{\mu}\right\|^{2}=\left(\Psi_{\mu}, J P J \Psi_{\mu}\right)=\left(P J \Psi_{\mu}, J \Psi_{\mu}\right)=\alpha$, we have

$$
\begin{aligned}
\alpha \bar{\mu}_{1}(A)+(1-\alpha) \bar{\mu}_{2}(A) & =\left(P^{\prime} \Psi_{\mu}, A \Psi_{\mu}\right)+\left(\left(I-P^{\prime}\right) \Psi_{\mu}, A \Psi_{\mu}\right) \\
& =\left(\Psi_{\mu}, A \Psi_{\mu}\right) \\
& =\bar{\mu}(A)
\end{aligned}
$$

for any $A \in \mathfrak{M}$. Moreover, since

$$
\begin{aligned}
\mathrm{e}^{-i t L} P^{\prime} \mathrm{e}^{i t L} & =\mathrm{e}^{-i t L} J P J \mathrm{e}^{i t L} \\
& =J \mathrm{e}^{-i t L} P \mathrm{e}^{i t L} J \\
& =J \sigma^{-t}(P) J=J P J=P^{\prime}
\end{aligned}
$$

and $\mathrm{e}^{i t L} \Psi_{\mu}=\Psi_{\mu}$, one easily checks that $\bar{\mu}_{1}$ and $\bar{\mu}_{2}$ are invariant and hence belong to $N\left(\mathfrak{M}, \tau^{t}\right)$. Let us assume that $\bar{\mu}_{1}=\bar{\mu}_{2}$. Then $\bar{\mu}=\bar{\mu}_{1}$ and for any $A \in \mathfrak{M}$ we get $\left(\alpha \Psi_{\mu}, A \Psi_{\mu}\right)=\left(P^{\prime} \Psi_{\mu}, A \Psi_{\mu}\right)$. It follows that

$$
0=\left[\mathfrak{M} \Psi_{\mu}\right]\left(P^{\prime}-\alpha\right) \Psi_{\mu}=\left[\mathfrak{M} \Psi_{\mu}\right] J(P-\alpha) J \Psi_{\mu}=J\left[\mathfrak{M}^{\prime} \Psi_{\mu}\right](P-\alpha) \Psi_{\mu}
$$

from which we conclude that $s_{\mu}(P-\alpha) \Psi_{\mu}=0$. Since $P \in \mathfrak{M}_{\sigma, \bar{\mu}}$ it satisfies $P=s_{\mu} P$ which yields $P \Psi_{\mu}=\alpha \Psi_{\mu}$. This is impossible since $P$ is a projection and $\alpha \in] 0,1\left[\right.$. This contradiction shows that the states $\bar{\mu}_{1} \neq \bar{\mu}_{2}$ provide a nontrivial decomposition of $\bar{\mu}$, a contradiction to its extremality. We conclude that $\bar{\mu}(P) \in\{0,1\}$ for all $P \in \mathfrak{M}_{\sigma, \bar{\mu}}$ and hence, by Proposition 8 , that $\left(\mathfrak{M}, \sigma^{t}, \bar{\mu}\right)$ is ergodic. Since this system is isomorphic to a normal form of $\left(\mathfrak{C}, \tau^{t}, \mu\right)$, the later is also ergodic by Proposition 7.

The last result in this subsection is the following characterization of the mixing property.

Proposition 11. Let $\left(\mathfrak{M}, \tau^{t}\right)$ be a $W^{*}$-dynamical system and $\mu \in N\left(\mathfrak{M}, \tau^{t}\right)$. The quantum dynamical system $\left(\mathfrak{M}, \tau^{t}, \mu\right)$ is mixing if and only if, for all $A, B \in \mathfrak{M}$, one has

$$
\lim _{t \rightarrow \infty} \mu\left(A s_{\mu} \tau^{t}(B)\right)=\mu(A) \mu(B) .
$$

Proof. By Remark 7 the system is mixing if and only if

$$
\lim _{t \rightarrow \infty} \nu \circ \tau^{t}\left(s_{\mu} B s_{\mu}\right)=\nu\left(s_{\mu}\right) \mu(B),
$$

holds for any $\nu \in \mathfrak{M}_{\star}$ and $B \in \mathfrak{M}$. If the system is mixing then Equ. (38) follows from Equ. (39) with $\nu \equiv \mu(A(\cdot)) \in \mathfrak{M}_{\star}$. 
To prove the reverse statement suppose that Equ. (38) holds. By Proposition 7 we may also assume that the system is in normal form, i.e., that $\mathfrak{M} \subset \mathfrak{B}(\mathcal{H}), \mu(B)=\left(\Omega_{\mu}, B \Omega_{\mu}\right)$ for some unit vector $\Omega_{\mu} \in \mathcal{H}, \overline{\mathfrak{M} \Omega_{\mu}}=\mathcal{H}$ and $s_{\mu}=\left[\mathfrak{M}^{\prime} \Omega_{\mu}\right]$.

Since $\tau^{t}$ is isometric it suffices to show Equ. (39) for a norm total set of $\nu$ in $\mathcal{S}(\mathfrak{M}, \mu)$. By Lemma $13, \mathcal{S}_{0}(\mathfrak{M}, \mu)$ is total in $\mathcal{S}(\mathfrak{M}, \mu)$ and its elements are of the form $\nu(B)=\left(R^{*} R \Omega_{\mu}, B \Omega_{\mu}\right)$ with $R \in \mathfrak{M}^{\prime}$. But $R^{*} R \Omega_{\mu}$ can be approximated in norm by vectors of the type $s_{\mu} A^{*} \Omega_{\mu}$ with $A \in \mathfrak{M}$ and hence the set of linear functionals of the form $\nu(B)=\left(s_{\mu} A^{*} \Omega_{\mu}, B \Omega_{\mu}\right)$ is also total in $\mathcal{S}(\mathfrak{M}, \mu)$. For such $\nu$, it does indeed follow from Equ. (38) that

$$
\nu \circ \tau^{t}\left(s_{\mu} B s_{\mu}\right)=\mu\left(A s_{\mu} \tau^{t}(B)\right) \rightarrow \mu(A) \mu(B)=\nu\left(s_{\mu}\right) \mu(B)
$$

as $t \rightarrow \infty$.

\subsection{Quantum Koopmanism}

To develop the spectral theory of quantum dynamical systems along the lines of Theorem 8 and 9 one may be tempted to use the $\mu$-Liouvillean $L_{\mu}$ or the standard Liouvillean $L$. The next exercise shows that this is not possible.

Exercise 24. In Examples 9, 14, assume that $E$ is a degenerate eigenvalue of $H$ and $\psi$ a corresponding normalized eigenvector. Show that the $\mu$-Liouvillean $L_{\mu}$ corresponding to the state $\mu(A)=(\psi, A \psi)$ is unitarily equivalent to $H-E$. Thus, even though $\mu$ is ergodic, 0 is not a simple eigenvalue of $L_{\mu}$. Show also that if $H$ has $N$ eigenvalues (counting multiplicities) then 0 is an $N$-fold degenerate eigenvalue of the standard Liouvillean $L$.

In fact a further reduction is necessary to obtain the analogue of the classical Liouvillean.

Theorem 16. Let $\left(\mathfrak{C}, \tau^{t}, \mu\right)$ be a quantum dynamical system with normal form $\left(\pi_{\mu}, \mathfrak{C}_{\mu}, \mathcal{H}_{\mu}, L_{\mu}, \Omega_{\mu}\right)$. Then the support $s_{\mu}=\left[\mathfrak{C}_{\mu}^{\prime} \Omega_{\mu}\right]$ reduces the $\mu$-Liouvillean $L_{\mu}$. We call reduced Liouvillean or simply "The Liouvillean" and denote by $\mathfrak{L}_{\mu}$ the restriction of $L_{\mu}$ to $\operatorname{Ran} s_{\mu}$.

(i) $\left(\mathfrak{C}, \tau^{t}, \mu\right)$ is ergodic if and only if Ker $\mathfrak{L}_{\mu}$ is one-dimensional.

(ii) $\left(\mathfrak{C}, \tau^{t}, \mu\right)$ is mixing if and only if

$$
\mathrm{w}-\lim _{t \rightarrow \infty} \mathrm{e}^{i t \mathfrak{L}_{\mu}}=\Omega_{\mu}\left(\Omega_{\mu}, \cdot\right) .
$$

(iii) If the spectrum of $\mathfrak{L}_{\mu}$ on $\left\{\Omega_{\mu}\right\}^{\perp}$ is purely absolutely continuous then $\left(\mathfrak{C}, \tau^{t}, \mu\right)$ is mixing.

Proof. Let $\hat{\tau}^{t}(A) \equiv \mathrm{e}^{i t L_{\mu}} A \mathrm{e}^{-i t L_{\mu}}$. Since the vector state $\hat{\mu}(A) \equiv\left(\Omega_{\mu}, A \Omega_{\mu}\right)$ is $\hat{\tau}^{t}$-invariant its support $s_{\mu}$ satisfies 


$$
\mathrm{e}^{i t L_{\mu}} s_{\mu} \mathrm{e}^{-i t L_{\mu}}=s_{\mu}
$$

and hence $L$ is reduced by $\operatorname{Ran} s_{\mu}$. As in the proof of Proposition 7 we can replace $\mathcal{S}(\mathfrak{C}, \mu)$ by $\mathcal{S}_{0}(\mathfrak{C}, \mu)$ in Definition 24 . For $\nu \in \mathcal{S}_{0}(\mathfrak{C}, \mu)$ we have

$$
\nu \circ \hat{\tau}^{t}(A)=\left(R \Omega_{\mu}, \mathrm{e}^{i t L_{\mu}} A \mathrm{e}^{-i t L_{\mu}} R \Omega_{\mu}\right)=\left(R^{*} R \Omega_{\mu}, \mathrm{e}^{i t L_{\mu}} A \Omega_{\mu}\right) .
$$

Since $R^{*} R \Omega_{\mu}=s_{\mu} R^{*} R \Omega_{\mu}$ we can rewrite

$$
\nu \circ \hat{\tau}^{t}(A)=\left(s_{\mu} R^{*} R \Omega_{\mu}, \mathrm{e}^{i t L_{\mu}} s_{\mu} A \Omega_{\mu}\right)=\left(s_{\mu} R^{*} R \Omega_{\mu}, \mathrm{e}^{i t \mathfrak{R}_{\mu}} s_{\mu} A \Omega_{\mu}\right) .
$$

From the mean ergodic theorem (Theorem 7) we get

$$
\lim _{T \rightarrow \infty} \frac{1}{T} \int_{0}^{T} \nu \circ \hat{\tau}^{t}(A) \mathrm{d} t=\left(s_{\mu} R^{*} R \Omega_{\mu}, P s_{\mu} A \Omega_{\mu}\right),
$$

where $P$ is the orthogonal projection on $\operatorname{Ker} \mathfrak{L}_{\mu}$. Note that if $P=\Omega_{\mu}\left(\Omega_{\mu}, \cdot\right)$ then the right hand side of the last formula reduces to

$$
\left(s_{\mu} R^{*} R \Omega_{\mu}, \Omega_{\mu}\right)\left(\Omega_{\mu}, s_{\mu} A \Omega_{\mu}\right)=\left(R \Omega_{\mu}, R \Omega_{\mu}\right)\left(\Omega_{\mu}, A \Omega_{\mu}\right)=\hat{\mu}(A) .
$$

Since the systems $\left\{s_{\mu} R^{*} R \Omega_{\mu} \mid R \in \mathfrak{C}_{\mu}^{\prime}\right\}$ and $\left\{s_{\mu} A \Omega_{\mu} \mid A \in \mathfrak{C}_{\mu}\right\}$ are both total in Ran $s_{\mu}$, we conclude that the system is ergodic if and only if $P=\Omega_{\mu}\left(\Omega_{\mu}, \cdot\right)$. Since $\Omega_{\mu}$ always belongs to the kernel of $\mathfrak{L}_{\mu}$, this is equivalent to the condition (i). The proof of (ii) is completely similar. Finally (iii) is proved as in the classical case (Theorem 9).

In many applications the invariant state $\hat{\mu}$ is faithful on $\mathfrak{C}_{\mu}$. In this case the $\mu$-Liouvillean and the standard Liouvillean coincide, $s_{\mu}=I$ and the previous result reduces to the simpler

Corollary 3. Let $\left(\mathfrak{C}, \tau^{t}, \mu\right)$ be a quantum dynamical system with normal form $\left(\pi_{\mu}, \mathfrak{C}_{\mu}, \mathcal{H}_{\mu}, L_{\mu}, \Omega_{\mu}\right)$ and assume that $s_{\mu}=\left[\mathfrak{C}_{\mu}^{\prime} \Omega_{\mu}\right]=I$.

(i) $\left(\mathfrak{C}, \tau^{t}, \mu\right)$ is ergodic if and only if $\operatorname{Ker} L_{\mu}$ is one-dimensional.

(ii) $\left(\mathfrak{C}, \tau^{t}, \mu\right)$ is mixing if and only if

$$
\mathrm{w}-\lim _{t \rightarrow \infty} \mathrm{e}^{i t L_{\mu}}=\Omega_{\mu}\left(\Omega_{\mu}, \cdot\right) .
$$

(iii) If the spectrum of $L_{\mu}$ on $\left\{\Omega_{\mu}\right\}^{\perp}$ is purely absolutely continuous then $\left(\mathfrak{C}, \tau^{t}, \mu\right)$ is mixing.

As in the classical case (see for example Theorem 9.12 in [5]), the spectrum of the Liouvillean of an ergodic quantum dynamical system is not arbitrary (see [24] and [31] for related results).

Theorem 17. Let $\mathfrak{L}_{\mu}$ be the Liouvillean of the ergodic quantum dynamical system $\left(\mathfrak{C}, \tau^{t}, \mu\right)$. 
(i) The set of eigenvalues of $\mathfrak{L}_{\mu}$ is a subgroup $\Sigma$ of the additive group $\mathbb{R}$.

(ii) The eigenvalues of $\mathfrak{L}_{\mu}$ are simple.

(iii) The spectrum of $\mathfrak{L}_{\mu}$ is invariant under translations in $\Sigma$, that is

$$
\sigma\left(\mathfrak{L}_{\mu}\right)+\Sigma=\sigma\left(\mathfrak{L}_{\mu}\right) .
$$

(iv) If $\Phi$ is a normalized eigenvector of $\mathfrak{L}_{\mu}$ then the corresponding vector state is the normal extension of $\mu$ to $\mathfrak{C}_{\mu}$.

(v) If $\left(\mathfrak{C}, \tau^{t}, \mu\right)$ is mixing then 0 is the only eigenvalue of $\mathfrak{L}_{\mu}$.

Proof. Let $\left(\pi_{\mu}, \mathfrak{C}_{\mu}, \mathcal{H}_{\mu}, L_{\mu}, \Omega_{\mu}\right)$ be the normal form of $\left(\mathfrak{C}, \tau^{t}, \mu\right)$ and set $\hat{\mu}(A)=\left(\Omega_{\mu}, A \Omega_{\mu}\right), \hat{\tau}^{t}(A)=\mathrm{e}^{i t L_{\mu}} A \mathrm{e}^{-i t L_{\mu}}, s_{\mu}=\left[\mathfrak{C}_{\mu}^{\prime} \Omega_{\mu}\right]$ and $\mathcal{K}_{\mu}=s_{\mu} \mathcal{H}_{\mu}$. Note that $\hat{\tau}^{t}$ extends to $\mathfrak{B}\left(\mathcal{H}_{\mu}\right)$ and that

$$
\hat{\tau}^{t}\left(\mathfrak{C}_{\mu}^{\prime}\right) \mathfrak{C}_{\mu}=\hat{\tau}^{t}\left(\mathfrak{C}_{\mu}^{\prime} \hat{\tau}^{-t}\left(\mathfrak{C}_{\mu}\right)\right)=\hat{\tau}^{t}\left(\hat{\tau}^{-t}\left(\mathfrak{C}_{\mu}\right) \mathfrak{C}_{\mu}^{\prime}\right)=\mathfrak{C}_{\mu} \hat{\tau}^{t}\left(\mathfrak{C}_{\mu}^{\prime}\right),
$$

shows that $\hat{\tau}^{t}\left(\mathfrak{C}_{\mu}^{\prime}\right)=\mathfrak{C}_{\mu}^{\prime}$. By Proposition $7,\left(\mathfrak{C}_{\mu}, \hat{\tau}^{t}, \hat{\mu}\right)$ is ergodic. Denote by $\Sigma$ the set of eigenvalues of $\mathfrak{L}_{\mu}$. If $\Sigma=\{0\}$ there is nothing to prove. Suppose that $\lambda \in \Sigma \backslash\{0\}$ and let $\Phi \in \mathcal{K}_{\mu}$ be a corresponding normalized eigenvector. Since $\mathrm{e}^{-i t L_{\mu} \Phi}=\mathrm{e}^{-i t \mathfrak{L}_{\mu}} \Phi=\mathrm{e}^{-i t \lambda} \Phi$ it follows that the state $\nu(A) \equiv(\Phi, A \Phi)$ is invariant. Moreover since

$$
\mathfrak{C}_{\mu}^{\prime} \Phi \subset \mathfrak{C}_{\mu}^{\prime} \mathcal{K}_{\mu}=\mathcal{K}_{\mu}
$$

one has $s_{\nu} \leq s_{\mu}$ and hence $\nu \ll \hat{\mu}$. Ergodicity yields that $\nu=\hat{\mu}$ and in particular $s_{\nu}=s_{\mu}$, i.e., $\overline{\mathfrak{C}_{\mu}^{\prime} \Phi}=\mathcal{K}_{\mu}$. This proves (iv).

For all $A \in \mathfrak{C}_{\mu}$ we have $\|A \Phi\|=\nu\left(A^{*} A\right)=\hat{\mu}\left(A^{*} A\right)=\left\|A \Omega_{\mu}\right\|$ which shows that the linear map

$$
\begin{aligned}
T_{\lambda}: \mathfrak{C}_{\mu} \Omega_{\mu} & \rightarrow \mathfrak{C}_{\mu} \Phi \\
A \Omega_{\mu} & \mapsto A \Phi,
\end{aligned}
$$

is well defined, densely defined and isometric on $\mathcal{H}_{\mu}$. We also denote by $T_{\lambda}$ its unique isometric extension to $\mathcal{H}_{\mu}$. For $A, B \in \mathfrak{C}_{\mu}$ we obtain

$$
\left(T_{\lambda}^{*} B \Phi, A \Omega_{\mu}\right)=\left(B \Phi, T_{\lambda} A \Omega_{\mu}\right)=(B \Phi, A \Phi)=\hat{\mu}\left(B^{*} A\right)=\left(B \Omega_{\mu}, A \Omega_{\mu}\right),
$$

from which we conclude that

$$
T_{\lambda}^{*} B \Phi=B \Omega_{\mu} .
$$

It follows that $\operatorname{Ran}\left(T_{\lambda}^{*}\right)^{\perp}=\operatorname{Ker} T_{\lambda}=\{0\}$, i.e., that $T_{\lambda}$ is unitary. Since $T_{\lambda} B A \Omega_{\mu}=B A \Phi=B T_{\lambda} A \Omega_{\mu}$ for all $A, B \in \mathfrak{C}_{\mu}$ we further get

$$
T_{\lambda} \in \mathfrak{C}_{\mu}^{\prime} .
$$

In particular, we have $s_{\mu} T_{\lambda}=T_{\lambda} s_{\mu}$, so that $T_{\lambda}$ and $T_{\lambda}^{*}$ map $\mathcal{K}_{\mu}$ into itself. Finally, for $A \in \mathfrak{C}_{\mu}$, we have 


$$
\begin{aligned}
\hat{\tau}^{t}\left(T_{\lambda}\right) A \Omega_{\mu} & =A \hat{\tau}^{t}\left(T_{\lambda}\right) \Omega_{\mu}=A \mathrm{e}^{i t L_{\mu}} T_{\lambda} \mathrm{e}^{-i t L_{\mu}} \Omega_{\mu} \\
& =A \mathrm{e}^{i t L_{\mu}} T_{\lambda} \Omega_{\mu}=A \mathrm{e}^{i t L_{\mu}} \Phi \\
& =\mathrm{e}^{i t \lambda} A \Phi=\mathrm{e}^{i t \lambda} T_{\lambda} A \Omega_{\mu},
\end{aligned}
$$

which yields

$$
\hat{\tau}^{t}\left(T_{\lambda}\right)=\mathrm{e}^{i t \lambda} T_{\lambda} .
$$

The last relation can be rewritten as $\mathrm{e}^{i t L_{\mu}}=T_{\lambda} \mathrm{e}^{i t\left(L_{\mu}+\lambda\right)} T_{\lambda}^{*}$ which means that $L_{\mu}+\lambda$ is unitarily equivalent to $L_{\mu}$. Property (iii) follows immediately.

To prove (ii) suppose that $\lambda$ is not a simple eigenvalue of $\mathfrak{L}_{\mu}$. Then there exists a unit vector $\Psi \in \mathcal{K}_{\mu}$ such that $\Psi \perp \Phi$ and $\mathfrak{L}_{\mu} \Psi=\lambda \Psi$. Since (41) implies that $T_{\lambda}^{*} \Phi=\Omega_{\mu}$ it follows that $T_{\lambda}^{*} \Psi \in \mathcal{K}_{\mu}$ is a unit vector orthogonal to $\Omega_{\mu}$. Moreover, Equ. (43) yields

$$
\mathrm{e}^{i t \mathfrak{L}_{\mu}} T_{\lambda}^{*} \Psi=\mathrm{e}^{i t L_{\mu}} T_{\lambda}^{*} \Psi=\hat{\tau}^{t}\left(T_{\lambda}\right)^{*} e^{i t L_{\mu}} \Psi=\left(e^{i t \lambda} T_{\lambda}\right)^{*} e^{i t \lambda} \Psi=T_{\lambda}^{*} \Psi,
$$

from which we conclude that $T_{\lambda}^{*} \Psi$ is an eigenvector of $\mathfrak{L}_{\mu}$ to the eigenvalue 0 , a contradiction to the simplicity of this eigenvalue. Thus, $\lambda$ is simple.

To prove (i) we first note that Equ. (43) yields that $\Phi^{\prime} \equiv T_{\lambda}^{*} \Omega_{\mu} \in$ $\mathcal{K}_{\mu}$ is an eigenvector of $\mathfrak{L}_{\mu}$ to the eigenvalue $-\lambda$. Second, suppose that $\lambda^{\prime} \in \Sigma \backslash\{0\}$ and let $T_{\lambda^{\prime}}$ be the corresponding unitary. We will then have $\hat{\tau}^{t}\left(T_{\lambda} T_{\lambda^{\prime}}\right)=\hat{\tau}^{t}\left(T_{\lambda}\right) \hat{\tau}^{t}\left(T_{\lambda^{\prime}}\right)=\mathrm{e}^{i t\left(\lambda+\lambda^{\prime}\right)} T_{\lambda} T_{\lambda^{\prime}}$ from which we can conclude again that $T_{\lambda} T_{\lambda^{\prime}} \Omega_{\mu}$ is an eigenvector of $\mathfrak{L}_{\mu}$ to the eigenvalue $\lambda+\lambda^{\prime}$.

To prove $(\mathrm{v})$ suppose on the contrary that the system is mixing and that $\mathfrak{L}_{\mu}$ has a non-zero eigenvalue $\lambda$. Let $\Phi \in \mathcal{K}_{\mu}$ be a corresponding normalized eigenvector. It follows that $\Phi \perp \Omega_{\mu}$ and

$$
\left(\Phi, \mathrm{e}^{i t \mathfrak{L}_{\mu}} \Phi\right)=\mathrm{e}^{i t \lambda},
$$

does not converge to zero as $t \rightarrow \infty$, a contradiction to the mixing criterion (ii) of Theorem 16 .

Using the relation between $\mu$-Liouvillean and standard Liouvillean described in Proposition 6 it is easy to rephrase Theorem 16 in terms of the standard Liouvillean.

Exercise 25. Let $\left(\mathfrak{C}, \tau^{t}, \mu\right)$ be a quantum dynamical system with standard form $(\eta, \mathfrak{M}, \mathcal{H}, J, \mathcal{C}, L, \Phi)$. Let $s_{\mu} \equiv\left[\mathfrak{M}^{\prime} \Phi\right] \in \mathfrak{M}$ be the support of the vector state $\bar{\mu}(A) \equiv(\Phi, A \Phi)$ and set $s_{\mu}^{\prime} \equiv J s_{\mu} J=[\mathfrak{M} \Phi] \in \mathfrak{M}^{\prime}$.

(i) Show that $S_{\mu} \equiv s_{\mu} s_{\mu}^{\prime}$ is an orthogonal projection which reduces the standard Liouvillean i.e., $\mathrm{e}^{i t L} S_{\mu} \mathrm{e}^{-i t L}=S_{\mu}$.

(ii) Show that the Liouvillean $\mathfrak{L}_{\mu}$ of Theorem 6 is unitarily equivalent to the restriction of the standard Liouvillean $L$ to $\operatorname{Ran} S_{\mu}$.

As we have seen, the $\mu$-Liouvillean is good enough to study the basic ergodic properties of a quantum dynamical system. However, to get deeper results on the structure of the state space and in particular on the manifold of invariant states it is necessary to use the standard Liouvillean. 
Theorem 18. Let $L$ be the standard Liouvillean of the $W^{*}$-dynamical system $\left(\mathfrak{M}, \tau^{t}\right)$. Then

$$
N\left(\mathfrak{M}, \tau^{t}\right)=\left\{\omega_{\Phi} \mid \Phi \in \operatorname{Ker} L,\|\Phi\|=1\right\},
$$

and in particular

(i) $\operatorname{Ker} L=\{0\}$ if and only if $N\left(\mathfrak{M}, \tau^{t}\right)=\varnothing$.

(ii) $\operatorname{Ker} L$ is one-dimensional if and only if $\left(\mathfrak{M}, \tau^{t}\right)$ has a unique normal invariant state. In this case, the corresponding quantum dynamical system is ergodic.

Proof. If $\mu \in N\left(\mathfrak{M}, \tau^{t}\right)$ then, by Proposition 13, $\mu$ has a standard vector representative $\Psi_{\mu}$ such that $\mathrm{e}^{-i t L} \Psi_{\mu}=\Psi_{\mu \circ \tau^{t}}=\Psi_{\mu}$ and hence $\Psi_{\mu} \in \operatorname{Ker} L$. Reciprocally, any unit vector $\Phi \in \operatorname{Ker} L$ defines a normal $\tau^{t}$-invariant state $\omega_{\Phi}$.

\subsection{Perturbation Theory}

Let $\left(\mathfrak{A}, \tau_{0}^{t}\right)$ be a $C^{*}$-dynamical system and denote by $\delta_{0}$ its generator. A local perturbation of the system is obtained by perturbing its generator with the bounded $*$-derivation associated with a self-adjoint element $V$ of $\mathfrak{A}$ :

$$
\delta_{V}=\delta+i[V, \cdot],
$$

with $\mathrm{D}\left(\delta_{V}\right)=\mathrm{D}(\delta)$. Using Theorem 2 it is easy to show that $\delta_{V}$ generates a strongly continuous group of $*$-automorphisms $\tau_{V}^{t}$ on $\mathfrak{A}$. In fact $\tau_{\lambda V}^{t}$ is an entire analytic function of $\lambda$. An expansion in powers of $\lambda$ is obtained by solving iteratively the integral equation (Duhamel formula)

$$
\tau_{\lambda V}^{t}(X)=\tau_{0}^{t}(X)+\lambda \int_{0}^{t} i\left[\tau_{0}^{t-s}(V), \tau_{\lambda V}^{s}(X)\right] \mathrm{d} s .
$$

The result is the Dyson-Robinson expansion

$$
\begin{aligned}
& \tau_{\lambda V}^{t}(X)=\tau_{0}^{t}(X)+ \\
& \quad+\sum_{N=1}^{\infty} \lambda^{N} \int_{0 \leqslant t_{1} \leqslant \cdots \leqslant t_{N} \leqslant t} i\left[\tau_{0}^{t_{1}}(V), i\left[\cdots, i\left[\tau_{0}^{t_{n}}(V), \tau_{0}^{t}(X)\right] \cdots\right]\right] \mathrm{d} t_{1} \cdots \mathrm{d} t_{N}
\end{aligned}
$$

which is norm convergent for any $\lambda \in \mathbb{C}, t \in \mathbb{R}$ and $X \in \mathfrak{A}$. Another useful representation of the locally perturbed dynamics is the interaction picture obtained through the Ansatz

$$
\tau_{V}^{t}(X)=\Gamma_{V}^{t} \tau_{0}^{t}(X) \Gamma_{V}^{t *} .
$$

It leads to the differential equation

$$
\partial_{t} \Gamma_{V}^{t}=i \Gamma_{V}^{t} \tau_{0}^{t}(V),
$$


with the initial condition $\Gamma_{V}^{0}=I$. It follows that $\Gamma_{V}^{t}$ is a unitary element of $\mathfrak{A}$ which has the norm convergent Araki-Dyson expansion

$$
\Gamma_{V}^{t} \equiv I+\sum_{N=1}^{\infty} i^{N} \int_{0 \leqslant t_{1} \leqslant \cdots \leqslant t_{N} \leqslant t} \tau_{0}^{t_{1}}(V) \cdots \tau_{0}^{t_{N}}(V) \mathrm{d} t_{1} \cdots \mathrm{d} t_{N}
$$

Moreover, $\Gamma_{V}^{t}$ satisfies the cocycle relations

$$
\Gamma_{V}^{t+s}=\Gamma_{V}^{t} \tau_{0}^{t}\left(\Gamma_{V}^{s}\right)=\tau_{V}^{t}\left(\Gamma_{V}^{s}\right) \Gamma_{V}^{t} .
$$

Note that the integrals in Equ. (44) and (46) are Riemann integrals of norm continuous $\mathfrak{A}$-valued functions.

The interaction picture allows to obtain unitary implementations of $\tau_{V}^{t}$ in an arbitrary representation $(\mathcal{H}, \pi)$ of $\mathfrak{A}$ carrying a unitary implementation $U_{0}^{t}$ of the unperturbed dynamics $\tau_{0}^{t}$. Indeed, one has

$$
\begin{aligned}
\pi\left(\tau_{V}^{t}(X)\right) & =\pi\left(\Gamma_{V}^{t} \tau_{0}^{t}(X) \Gamma_{V}^{t *}\right) \\
& =\pi\left(\Gamma_{V}^{t}\right) \pi\left(\tau_{0}^{t}(X)\right) \pi\left(\Gamma_{V}^{t}\right)^{*} \\
& =\pi\left(\Gamma_{V}^{t}\right) U_{0}^{t} \pi(X) U_{0}^{t *} \pi\left(\Gamma_{V}^{t}\right)^{*}
\end{aligned}
$$

from which we conclude that the unitary $U_{V}^{t}=\pi\left(\Gamma_{V}^{t}\right) U_{0}^{t}$ implements $\tau_{V}^{t}$ in $\mathcal{H}$. The cocycle property (47) shows that $U_{V}^{t}$ has the group property. From the expansion (46) we get norm convergent expansion (the integral are in the strong Riemann sense)

$$
\begin{aligned}
& U_{V}^{t}=U_{0}^{t}+ \\
& +\sum_{N=1}^{\infty} i^{N} \int_{0 \leqslant t_{1} \leqslant \cdots \leqslant t_{N} \leqslant t} U_{0}^{t_{1}} \pi(V) U_{0}^{t_{2}-t_{1}} \cdots U_{0}^{t_{N}-t_{N-1}} \pi(V) U_{0}^{t-t_{N}} \mathrm{~d} t_{1} \cdots \mathrm{d} t_{N} .
\end{aligned}
$$

Let $G_{V}$ be the self-adjoint generator of $U_{V}^{t}$. Applying the last formula to a vector $\Phi \in \mathrm{D}\left(G_{0}\right)$ and differentiating at $t=0$ we obtain $\Phi \in \mathrm{D}\left(G_{V}\right)$ and

$$
G_{V}=G_{0}+\pi(V)
$$

Note however that the unitary implementation of $\tau_{V}^{t}$ in $\mathcal{H}$ is by no means unique. Indeed, $\mathrm{e}^{i t K}$ is another implementation if and only if

$$
\mathrm{e}^{i t G_{V}} \pi(X) \mathrm{e}^{-i t G_{V}}=\mathrm{e}^{i t K} \pi(X) \mathrm{e}^{-i t K},
$$

for all $X \in \mathfrak{A}$ and all $t$. Thus $\mathrm{e}^{-i t K} \mathrm{e}^{i t G_{V}}$ must be a unitary element of $\pi(\mathfrak{A})^{\prime}$ for all $t$. Assuming that $\mathrm{D}(K)=\mathrm{D}\left(G_{V}\right)$, differentiation at $t=0$ yields

$$
K=G_{V}-W=G_{0}+\pi(V)-W,
$$

for some self-adjoint element $W \in \pi(\mathfrak{A})^{\prime}$. Then $\Gamma_{W}^{\prime t}=\mathrm{e}^{i t K} \mathrm{e}^{-i t G_{V}}$ satisfies the differential equation 


$$
\partial_{t} \Gamma_{W}^{\prime t}=-i \Gamma_{W}^{\prime t} W^{t}
$$

with $W^{t}=\mathrm{e}^{i t G_{V}} W \mathrm{e}^{-i t G_{V}}$ and initial value $\Gamma_{W}^{\prime 0}=I$. Since $\mathrm{e}^{i t G_{V}}=\pi\left(\Gamma_{V}^{t}\right) \mathrm{e}^{i t G_{0}}$ and $\mathrm{e}^{i t G_{0}} W \mathrm{e}^{-i t G_{0}} \in \pi(\mathfrak{A})^{\prime}$ for all $t$, we have $W^{t}=\mathrm{e}^{i t G_{0}} W \mathrm{e}^{-i t G_{0}}$ and $\Gamma_{W}^{\prime t}$ is given by the norm convergent expansion

$$
\Gamma_{W}^{\prime t}=I+\sum_{N=1}^{\infty}(-i)^{N} \int_{0 \leqslant t_{1} \leqslant \cdots \leqslant t_{N} \leqslant t} W^{t_{1}} \cdots W^{t_{N}} \mathrm{~d} t_{1} \cdots \mathrm{d} t_{N} .
$$

Local perturbations of $W^{*}$-dynamical systems can be treated in a completely similar way, the only change is that the norm topology should be replaced with the $\sigma$-weak topology, i.e. the integrals in Equ. (44) and (46) have to be understood in the weak- $\star$ sense.

Unbounded perturbations of a $W^{*}$-dynamical systems $\left(\mathfrak{M}, \tau_{0}^{t}\right)$ are common in applications. They require a slightly more sophisticated treatment. I shall only consider the case of a unitarily implemented unperturbed dynamics ${ }^{4}$

$$
\tau_{0}^{t}(X)=\mathrm{e}^{i t G_{0}} X \mathrm{e}^{-i t G_{0}} .
$$

Let $V$ be a self-adjoint operator affiliated to $\mathfrak{M}$, i.e., $\mathrm{e}^{i t V} \in \mathfrak{M}$ for all $t \in \mathbb{R}$, and such that $G_{0}+V$ is essentially self-adjoint on $\mathrm{D}\left(G_{0}\right) \cap \mathrm{D}(V)$. Denote by $G_{V}$ its self-adjoint extension and set

$$
\tau_{V}^{t}(X)=\mathrm{e}^{i t G_{V}} X \mathrm{e}^{-i t G_{V}} .
$$

Defining the unitary

$$
\Gamma_{V}^{t}=\mathrm{e}^{i t G_{V}} \mathrm{e}^{-i t G_{0}},
$$

the Trotter product formula (Theorem 15 in Lecture [32]) shows that

$$
\Gamma_{V}^{t}=\mathrm{s}-\lim _{n \rightarrow \infty} \mathrm{e}^{i t V / n} \tau_{0}^{t / n}\left(\mathrm{e}^{i t V / n}\right) \cdots \tau_{0}^{(n-1) t / n}\left(\mathrm{e}^{i t V / n}\right),
$$

and since $\mathrm{e}^{i t V / n} \in \mathfrak{M}$ we get $\Gamma_{V}^{t} \in \mathfrak{M}$. By construction Equ. (45) and (47) remain valid. In particular $\tau_{V}^{t}$ leaves $\mathfrak{M}$ invariant and $\left(\mathfrak{M}, \tau_{V}^{t}\right)$ is a $W^{*}$-dynamical system. Another application of the Trotter formula further gives, for $X^{\prime} \in \mathfrak{M}^{\prime}$,

$$
\mathrm{e}^{i t G_{V}} X^{\prime} \mathrm{e}^{-i t G_{V}}=\mathrm{s}-\lim _{n \rightarrow \infty}\left(\mathrm{e}^{i t G_{0} / n} \mathrm{e}^{i t V / n}\right)^{n} X^{\prime}\left(\mathrm{e}^{-i t V / n} \mathrm{e}^{-i t G_{0} / n}\right)^{n},
$$

which allows to conclude that

$$
\mathrm{e}^{i t G_{V}} X^{\prime} \mathrm{e}^{-i t G_{V}}=\mathrm{e}^{i t G_{0}} X^{\prime} \mathrm{e}^{-i t G_{0}},
$$

for all $X^{\prime} \in \mathfrak{M}^{\prime}$.

\footnotetext{
${ }^{4}$ This is not a real restriction since it is always possible to go to a standard representation where such an implementation always exists.
} 
As in the $C^{*}$-case, other implementations of $\tau_{V}^{t}$ can be obtained by choosing a self-adjoint $W$ affiliated to $\mathfrak{M}^{\prime}$ and such that $G-W$ is essentially selfadjoint on $\mathrm{D}(G) \cap \mathrm{D}(W)$. Denoting by $G_{W}^{\prime}$ its self-adjoint extension we set

$$
\Gamma_{W}^{\prime t}=\mathrm{e}^{i t G_{W}^{\prime}} \mathrm{e}^{-i t G}
$$

By the Trotter formula argument we get $\Gamma_{W}^{\prime t} \in \mathfrak{M}^{\prime}$ and Equ. (49) gives

$$
\begin{aligned}
\left(\Gamma_{W}^{\prime t} \Gamma_{V}^{t} \mathrm{e}^{i t G}\right)\left(\Gamma_{W}^{\prime s} \Gamma_{V}^{s} \mathrm{e}^{i s G}\right) & =\Gamma_{W}^{\prime t} \mathrm{e}^{i t G_{V}} \Gamma_{W}^{\prime s} \mathrm{e}^{-i t G_{V}} \mathrm{e}^{i(t+s) G_{V}} \\
& =\Gamma_{W}^{\prime t} \mathrm{e}^{i t G} \Gamma_{W}^{\prime s} \mathrm{e}^{-i t G} \mathrm{e}^{i(t+s) G_{V}} \\
& =\Gamma_{W}^{\prime t+s} \mathrm{e}^{i(t+s) G_{V}} \\
& =\Gamma_{W}^{\prime t+s} \Gamma_{V}^{t+s} \mathrm{e}^{i(t+s) G}
\end{aligned}
$$

Thus $\Gamma_{W}^{\prime t} \Gamma_{V}^{t} \mathrm{e}^{i t G}=\mathrm{e}^{i t G_{W}^{\prime}} \mathrm{e}^{-i t G} \mathrm{e}^{i t G_{V}}$ is a unitary group, and differentiation shows that its generator is given on $\mathrm{D}(G) \cap \mathrm{D}(V) \cap \mathrm{D}(W)$ by $G+V-W$.

As an application let me now derive the perturbation formula for the standard Liouvillean.

Theorem 19. Let $L_{0}$ be the standard Liouvillean of the $W^{*}$-dynamical system $\left(\mathfrak{M}, \tau^{t}\right)$ which we suppose to be in standard form $(\mathfrak{M}, \mathcal{H}, J, \mathcal{C})$. Let $V$ be a selfadjoint operator affiliated to $\mathfrak{M}$ and such that

(i) $L_{0}+V$ is essentially self-adjoint on $\mathrm{D}\left(L_{0}\right) \cap \mathrm{D}(V)$.

(ii) $L_{0}+V-J V J$ essentially self-adjoint on $\mathrm{D}\left(L_{0}\right) \cap \mathrm{D}(V) \cap \mathrm{D}(J V J)$.

Denote by $L_{V}$ the self-adjoint extension of $L_{0}+V-J V J$. Then $L_{V}$ is the standard Liouvillean of the perturbed dynamical system $\left(\mathfrak{M}, \tau_{V}^{t}\right)$.

Proof. $W=J V J$ with $\mathrm{D}(W)=J \mathrm{D}(V)$ is affiliated to $\mathfrak{M}^{\prime}$ since

$$
\mathrm{e}^{i t W}=J \mathrm{e}^{-i t V} J \in \mathfrak{M}^{\prime} .
$$

Furthermore from Equ. (30) we get $\mathrm{D}\left(L_{0}\right)=J \mathrm{D}\left(L_{0}\right)$ and

$$
L_{0}-W=-J\left(L_{0}+V\right) J,
$$

which is essentially self-adjoint on $\mathrm{D}\left(L_{0}\right) \cap \mathrm{D}(W)=J\left(\mathrm{D}\left(L_{0}\right) \cap \mathrm{D}(V)\right)$. Thus

$$
\Gamma_{W}^{\prime t}=J \Gamma_{V}^{t} J
$$

and since $L_{V}$ is essentially self-adjoint on $\mathrm{D}\left(L_{0}\right) \cap \mathrm{D}(V) \cap \mathrm{D}(W)$ we get from the above discussion

$$
\mathrm{e}^{i t L_{V}}=\Gamma_{V}^{t} J \Gamma_{V}^{t} J \mathrm{e}^{i t L_{0}} .
$$

The unitary group $\mathrm{e}^{i t L_{V}}$ implements $\tau_{V}^{t}$ and leaves the cone $\mathcal{C}$ invariant by property (iii) of Definition 18. This show that $L_{V}$ is the standard Liouvillean of the perturbed system. 


\section{KMS States}

\subsection{Definition and Basic Properties}

For a quantum system with an Hamiltonian $H$ such that $\operatorname{Tr} \mathrm{e}^{-\beta H}<\infty$, the Gibbs-Boltzmann prescription for the canonical thermal equilibrium ensemble at inverse temperature $\beta=1 / k_{B} T$ is the density matrix

$$
\rho_{\beta}=Z_{\beta}^{-1} \mathrm{e}^{-\beta H},
$$

where the normalization factor $Z_{\beta}=\operatorname{Tr} \mathrm{e}^{-\beta H}$ is the canonical partition function (see Section 3 in [33]). On the other hand the dynamics is given by the spatial automorphisms

$$
\tau^{t}(A)=\mathrm{e}^{i t H} A \mathrm{e}^{-i t H} .
$$

To avoid unnecessary technical problems, think of $H$ as being bounded. The fact that the semi-group entering Equ. (50) reappears, after analytic continuation to the imaginary axis, in Equ. (51) expresses the very strong coupling that exists in quantum mechanics between dynamics and thermal equilibrium. To formalize this remark let us consider, for two observables $A, B$, the equilibrium correlation function

$$
\omega\left(A \tau^{t}(B)\right)=Z_{\beta}^{-1} \operatorname{Tr}\left(\mathrm{e}^{-\beta H} A \mathrm{e}^{i t H} B \mathrm{e}^{-i t H}\right)=Z_{\beta}^{-1} \operatorname{Tr}\left(\mathrm{e}^{-i(t-i \beta) H} A \mathrm{e}^{i t H} B\right) .
$$

Using the cyclicity of the trace, analytic continuation of this function to $t+i \beta$ gives

$$
\omega\left(A \tau^{t+i \beta}(B)\right)=Z_{\beta}^{-1} \operatorname{Tr}\left(\mathrm{e}^{-i t H} A \mathrm{e}^{i(t+i \beta) H} B\right)=\omega\left(\tau^{t}(B) A\right) .
$$

Thus there is a function $F_{\beta}(A, B ; z)$, analytic in the strip

$$
S_{\beta} \equiv\{z \in \mathbb{C} \mid 0<\operatorname{Im} z<\beta\}
$$

and taking boundary values

$$
\begin{aligned}
F_{\beta}(A, B ; t) & =\omega\left(A \tau^{t}(B)\right), \\
F_{\beta}(A, B ; t+i \beta) & =\omega\left(\tau^{t}(B) A\right),
\end{aligned}
$$

on $\partial S_{\beta}$. These are the so called KMS boundary conditions. In some sense, the analytic function $F_{\beta}(A, B ; z)$ encodes the non-commutativity of the product $A B$ in the state $\rho_{\beta}$. A KMS state is a state for which such a function, satisfying the KMS boundary condition, exists for all observables $A, B$.

Definition 25. Let $\left(\mathfrak{C}, \tau^{t}\right)$ be a $C^{*}$ - or $W^{*}$-dynamical system. A state $\omega$ on $\mathfrak{C}$, assumed to be normal in the $W^{*}$-case, is said to be a $\left(\tau^{t}, \beta\right)$-KMS state for $\beta>0$ if for any $A, B \in \mathfrak{C}$ there exists a function $F_{\beta}(A, B ; z)$ analytic in the strip $S_{\beta}$, continuous on its closure and satisfying the KMS conditions (52) and (53) on its boundary. 
Remark 8. $i$. KMS states for negative values of $\beta$ have no physical meaning (except for very special systems). However, for historical reasons, they are widely used in the mathematical literature. These states have the same definition, the strip $S_{\beta}$ being replaced by $S_{\beta}=\{z \in \mathbb{C} \mid \beta<\operatorname{Im} z<0\}$.

ii. Our definition excludes the degenerate case $\beta=0$. The KMS boundary condition then becomes $\omega(A B)=\omega(B A)$ for all $A, B \in \mathfrak{C}$. Such a state is called a trace.

iii. If $\omega$ is a $\left(\tau^{t}, \beta\right)$-KMS state then it is also $\left(\tau^{\gamma t}, \beta / \gamma\right)$-KMS. Note however that there is no simple connection between KMS states at different temperatures for the same group $\tau^{t}$.

If $\left(\mathfrak{C}, \tau^{t}\right)$ is a $C^{*}$ - or $W^{*}$-dynamical system, an element $A \in \mathfrak{C}$ is analytic for $\tau^{t}$ if the function $t \mapsto \tau^{t}(A)$, defined for $t \in \mathbb{R}$, extends to an entire analytic function $z \mapsto \tau^{z}(A)$ of $z \in \mathbb{C}$. Let us denote by $\mathfrak{C}_{\tau}$ the set of analytic elements for $\tau^{t}$. It is easy to see that $\mathfrak{C}_{\tau}$ is a $*$-subalgebra of $\mathfrak{C}$. Indeed, $\tau^{z}(A+B)=\tau^{z}(A)+\tau^{z}(B)$ and $\tau^{z}(A B)=\tau^{z}(A) \tau^{z}(B)$ hold for $z \in \mathbb{R}$ and hence extend to all $z \in \mathbb{C}$ by analytic continuation. Moreover, if $\tau^{z}(A)$ is entire analytic then so is $\tau^{z}\left(A^{*}\right) \equiv \tau^{\bar{z}}(A)^{*}$. For $A \in \mathfrak{C}$, set

$$
A_{n} \equiv \sqrt{\frac{n}{\pi}} \int_{-\infty}^{\infty} \tau^{t}(A) \mathrm{e}^{-n t^{2}} \mathrm{~d} t,
$$

where the integral is understood in the Riemann sense in the $C^{*}$-case and in the weak- $\star$ sense in the $W^{*}$-case. One has $A_{n} \in \mathfrak{C}$ with $\left\|A_{n}\right\| \leq\|A\|$ and the formula

$$
\tau^{z}\left(A_{n}\right)=\sqrt{\frac{n}{\pi}} \int_{-\infty}^{\infty} \tau^{t}(A) \mathrm{e}^{-n(t-z)^{2}} \mathrm{~d} t,
$$

clearly shows that $\tau^{z}\left(A_{n}\right)$ is an entire analytic function of $z$ such that

$$
\left\|\tau^{z}\left(A_{n}\right)\right\| \leq \mathrm{e}^{n \operatorname{Im}(z)^{2}}\|A\|,
$$

and hence $A_{n} \in \mathfrak{C}_{\tau}$. Finally, since

$$
A_{n}-A=\int_{-\infty}^{\infty}\left(\tau^{t / \sqrt{n}}(A)-A\right) \mathrm{e}^{-t^{2}} \frac{\mathrm{d} t}{\sqrt{\pi}},
$$

it follows from Lebesgue dominated convergence theorem that the sequence $A_{n}$ converges towards $A$ in norm in the $C^{*}$-case and $\sigma$-weakly in the $W^{*}$-case. This shows that the $*$-subalgebra $\mathfrak{C}_{\tau}$ is dense in $\mathfrak{C}$ in the appropriate topology.

Let $\omega$ be a $\left(\tau^{t}, \beta\right)$-KMS state on $\mathfrak{C}, A \in \mathfrak{C}$ and $B \in \mathfrak{C}_{\tau}$. Then the function $G(z) \equiv \omega\left(A \tau^{z}(B)\right)$ is entire analytic and for $t \in \mathbb{R}$ one has $G(t)=F_{\beta}(A, B ; t)$. Thus, the function $G(z)-F_{\beta}(A, B ; z)$ is analytic on $S_{\beta}$, continuous on $S_{\beta} \cup \mathbb{R}$ and vanishes on $\mathbb{R}$. By the Schwarz reflection principle it extends to an analytic function on the strip $\{z \mid-\beta<\operatorname{Im} z<\beta\}$ which vanishes on $\mathbb{R}$ and therefore on the entire strip. By continuity

$$
F_{\beta}(A, B ; z)=\omega\left(A \tau^{z}(B)\right),
$$


holds for $z \in \overline{S_{\beta}}$ and in particular one has

$$
\omega\left(A \tau^{i \beta}(B)\right)=\omega(B A) .
$$

As a first consequence of this fact, let us prove the most important property of KMS states.

Theorem 20. If $\omega$ is a $\left(\tau^{t}, \beta\right)$-KMS state then it is $\tau^{t}$-invariant.

Proof. For $A \in \mathfrak{C}_{\tau}$, the function $f(z)=\omega\left(\tau^{z}(A)\right)$ is entire analytic. Moreover, Equ. (54) shows that it is $i \beta$-periodic

$$
f(z+i \beta)=\omega\left(I \tau^{i \beta}\left(\tau^{z}(A)\right)\right)=\omega\left(\tau^{z}(A) I\right)=f(z) .
$$

On the closed strip $\overline{S_{\beta}}$ the estimate

$$
|f(t+i \alpha)| \leqslant\left\|\tau^{t}\left(\tau^{i \alpha}(A)\right)\right\|=\left\|\tau^{i \alpha}(A)\right\| \leqslant \sup _{0 \leqslant \gamma \leqslant \beta}\left\|\tau^{i \gamma}(A)\right\|<\infty
$$

holds and therefore $f$ is bounded on whole complex plane. By Liouville Theorem, $f$ is constant. By continuity, this property extends to all observables.

As shown by the next result, Property (54) characterizes KMS states in a way that is often more convenient than Definition 25 (See Proposition 5.3.7 in $[12])$.

Theorem 21. A state $\omega$ on a $C^{*}$ - (resp. $\left.W^{*}-\right)$ algebra $\mathfrak{C}^{*}$ is $\left(\tau^{t}, \beta\right)-K M S$ if and only it is normal in the $W^{*}$-case and there exists a norm dense (resp. $\sigma$-weakly dense), $\tau^{t}$-invariant $*$-subalgebra $\mathfrak{D}$ of analytic elements for $\tau^{t}$ such that

$$
\omega\left(A \tau^{i \beta}(B)\right)=\omega(B A)
$$

holds for all $A, B \in \mathfrak{D}$.

Proof. It remains to prove sufficiency. We will only consider the $C^{*}$-case and refer the reader to the proof of Proposition 5.3.7 in [12] for the $W^{*}$-case. For $A, B \in \mathfrak{D}$, the function defined by

$$
F_{\beta}(A, B ; z) \equiv \omega\left(A \tau^{z}(B)\right)
$$

is analytic on the strip $S_{\beta}$ and continuous on its closure. Since $\mathfrak{D}$ is invariant under $\tau^{t}$ one has $\tau^{t}(B) \in \mathfrak{D}$ and hence

$$
\begin{aligned}
F_{\beta}(A, B ; t) & =\omega\left(A \tau^{t}(B)\right), \\
F_{\beta}(A, B ; t+i \beta) & =\omega\left(A \tau^{i \beta}\left(\tau^{t}(B)\right)\right)=\omega\left(\tau^{t}(B) A\right) .
\end{aligned}
$$

From the bound $\left|\omega\left(A \tau^{z}(B)\right)\right| \leq\|A\|\left\|\tau^{i \operatorname{Im} z}(B)\right\|$ we deduce that $F_{\beta}(A, B ; z)$ is bounded on $\overline{S_{\beta}}$ and Hadamard tree line theorem yields 


$$
\sup _{z \in \overline{S_{\beta}}}\left|F_{\beta}(A, B ; z)\right| \leq\|A\|\|B\| .
$$

Since $\mathfrak{D} \subset \mathfrak{C}_{\tau}$ is norm dense, any $A, B \in \mathfrak{C}$ can be approximated by sequences $A_{n}, B_{n} \in \mathfrak{D}$. From the identity

$F_{\beta}\left(A_{n}, B_{n} ; z\right)-F_{\beta}\left(A_{m}, B_{m} ; z\right)=F_{\beta}\left(A_{n}-A_{m}, B_{n} ; z\right)+F_{\beta}\left(A_{m}, B_{n}-B_{m} ; z\right)$,

and the bound (55) we conclude that the sequence $F_{\beta}\left(A_{n}, B_{n} ; z\right)$ is uniformly Cauchy in $\overline{S_{\beta}}$. Its limit, which we denote by $F_{\beta}(A, B ; z)$, is therefore analytic on $S_{\beta}$ and continuous on its closure where it satisfies (55). Finally, for $t \in \mathbb{R}$ one has

$$
\begin{aligned}
F_{\beta}(A, B ; t) & =\lim _{n} \omega\left(A_{n} \tau^{t}\left(B_{n}\right)\right)=\omega\left(A \tau^{t}(B)\right), \\
F_{\beta}(A, B ; t+i \beta) & =\lim _{n} \omega\left(\tau^{t}\left(B_{n}\right) A_{n}\right)=\omega\left(\tau^{t}(B) A\right),
\end{aligned}
$$

which concludes the proof.

Example 15. (Finite quantum systems, continuation of Example 5) Let $H$ be a self-adjoint operator on the Hilbert space $\mathcal{H}$ and consider the induced $W^{*}$ dynamical system $\left(\mathfrak{B}(\mathcal{H}), \tau^{t}\right)$. If $\omega$ is a $(\tau, \beta)$-KMS state then there exists a density matrix $\rho$ on $\mathcal{H}$ such that $\omega(A)=\operatorname{Tr}(\rho A)$ for all $A \in \mathfrak{B}(\mathcal{H})$. Relation (54) with $A \equiv \phi(\psi, \cdot)$ yields

$$
\left(\psi, \tau^{i \beta}(B) \rho \phi\right)=(\psi, \rho B \phi),
$$

and hence $\tau^{i \beta}(B) \rho=\rho B$ for all $\tau^{t}$-analytic elements $B$. If $\phi$ and $\psi$ belong to the dense subspace of entire analytic vectors for $\mathrm{e}^{i t H}$ then $B \equiv \phi(\psi, \cdot)$ is analytic for $\tau^{t}$ and $\tau^{i \beta}(B)=\mathrm{e}^{-\beta H} \phi\left(\mathrm{e}^{\beta H} \psi, \cdot\right)$. For $\psi \neq 0$ we can rewrite the relation $\tau^{i \beta}(B) \rho \psi=\rho B \psi$ as

$$
\rho \phi=\frac{\left(\mathrm{e}^{\beta H} \psi, \rho \psi\right)}{(\psi, \psi)} \mathrm{e}^{-\beta H} \phi,
$$

from which we can conclude that

$$
\rho=\frac{\mathrm{e}^{-\beta H}}{\operatorname{Tr} \mathrm{e}^{-\beta H}} .
$$

Thus, the $W^{*}$-dynamical system $\left(\mathfrak{B}(\mathcal{H}), \tau^{t}\right)$ admits a $\left(\tau^{t}, \beta\right)$-KMS state if and only if $\mathrm{e}^{-\beta H}$ is trace class. Moreover, if such a state exists then it is unique.

Example 16. (Ideal Fermi gas) Let $\omega_{\beta}$ be a $\left(\tau^{t}, \beta\right)$-KMS state for the $C^{*}$ dynamical system of Example 2. Then $\omega_{\beta}\left(a^{*}(g) a(f)\right)$ is a sesquilinear form on $\mathfrak{h}$. Since

$$
\mid \omega_{\beta}\left(a^{*}(g) a(f) \mid \leqslant\left\|a^{*}(g)\right\|\|a(f)\| \leqslant\|g\|\|f\|,\right.
$$

there exists a bounded self-adjoint $T$ on $\mathfrak{h}$ such that 


$$
\omega_{\beta}\left(a^{*}(g) a(f)\right)=(f, T g) .
$$

Moreover, since $\omega_{\beta}\left(a^{*}(f) a(f)\right) \geqslant 0, T$ satisfies the inequalities $0 \leqslant T \leqslant I$. For $t \in \mathbb{R}$ one has $\omega_{\beta}\left(a^{*}(g) \tau^{t}(a(f))\right)=\left(\mathrm{e}^{i t h} f, T g\right)$ and for $f$ in the dense subspace of entire analytic vectors for $h$ the analytic continuation of this function to $t+i \beta$ is given by $\left(\mathrm{e}^{\beta h} \mathrm{e}^{i t h} f, T g\right)=\left(T \mathrm{e}^{\beta h} \mathrm{e}^{i t h} f, g\right)$. The CAR gives $\tau^{t}(a(f)) a^{*}(g)=-a^{*}(g) \tau^{t}(a(f))+\left(\mathrm{e}^{i t h} f, g\right)$ and hence

$$
\omega_{\beta}\left(\tau^{t}(a(f)) a^{*}(g)\right)=\left(\mathrm{e}^{i t h} f, g\right)-\omega_{\beta}\left(a^{*}(g) a\left(\mathrm{e}^{i t h} f\right)\right)=\left((I-T) \mathrm{e}^{i t h} f, g\right),
$$

and the KMS boundary condition implies $T \mathrm{e}^{\beta h}=I-T$ from which we conclude that

$$
T=\frac{1}{1+\mathrm{e}^{\beta h}} .
$$

Consider now the expectation of an arbitrary even monomial $(m+n$ even)

$$
W_{m, n}\left(g_{1}, \ldots g_{m} ; f_{1}, \ldots f_{n}\right)=\omega_{\beta}\left(a^{*}\left(g_{m}\right) \cdots a^{*}\left(g_{1}\right) a\left(f_{1}\right) \cdots a\left(f_{n}\right)\right) .
$$

We first show that $W_{m, 0}=W_{0, n}=0$. For any $f$ in the dense subspace of entire analytic vectors for $h$, the KMS condition and the CAR lead to

$$
W_{0, n}\left(f, f_{2}, \ldots f_{n}\right)=W_{0, n}\left(f_{2}, \ldots f_{n}, \mathrm{e}^{\beta h} f\right)=(-1)^{n-1} W_{0, n}\left(\mathrm{e}^{\beta h} f, f_{2}, \ldots f_{n}\right),
$$

from which we get $W_{0, n}\left(\left(1+\mathrm{e}^{\beta h}\right) f, f_{2}, \ldots f_{n}\right)=0$. If $g$ is an entire analytic vector for $h$, so is $f=\left(1+\mathrm{e}^{\beta h}\right)^{-1} g$ and we can conclude that $W_{0, n}\left(g, f_{2}, \cdots, f_{n}\right)=0$ for all $g$ in a dense subspace and all $f_{2}, \ldots f_{n} \in \mathfrak{h}$ and hence for all $g, f_{2}, \ldots f_{n} \in \mathfrak{h}$. By conjugation, this implies that for all $g_{1}, \ldots g_{m} \in \mathfrak{h}$ one also has $W_{m, 0}\left(g_{1}, \ldots g_{m}\right)=0$.

We consider now the general case. Using the KMS boundary condition, we can write

$$
W_{m, n}\left(g_{1}, \ldots ; f_{1}, \ldots\right)=\omega_{\beta}\left(a\left(\mathrm{e}^{-\beta h} f_{n}\right) a^{*}\left(g_{m}\right) \cdots a^{*}\left(g_{1}\right) a\left(f_{1}\right) \cdots a\left(f_{n-1}\right)\right) .
$$

Using the CAR we can commute back the first factor through the others to bring it again in the last position. This leads to the formula

$$
\begin{gathered}
W_{m, n}\left(g_{1}, \ldots g_{m} ; f_{1}, \ldots f_{n}\right)=-W_{m, n}\left(g_{1}, \ldots g_{m} ; f_{1}, \ldots \mathrm{e}^{-\beta h} f_{n}\right)+ \\
+\sum_{j=1}^{m}(-1)^{m-j}\left(g_{j}, \mathrm{e}^{-\beta h} f_{n}\right) W_{m-1, n-1}\left(g_{1}, \ldots g_{j-1}, g_{j+1}, \ldots g_{m} ; f_{1}, \ldots f_{n-1}\right) .
\end{gathered}
$$

Replacing $f_{n}$ by $\left(1+\mathrm{e}^{-\beta h}\right)^{-1} f_{n}$ and using Equ. (56), this can be rewritten as

$$
\begin{aligned}
& W_{m, n}\left(g_{1}, \ldots g_{m} ; f_{1}, \ldots f_{n}\right)= \\
& \quad \sum_{j=1}^{m}(-1)^{m-j} W_{1,1}\left(g_{j} ; f_{n}\right) W_{m-1, n-1}\left(g_{1}, \ldots g_{j-1}, g_{j+1}, \ldots g_{m} ; f_{1}, \ldots f_{n-1}\right) .
\end{aligned}
$$


Iteration of this formula shows that $W_{m, n}=0$ if $m \neq n$, and $W_{n, n}$ can be expressed as sum of products of $W_{1,1}\left(g_{j} ; f_{k}\right)=\left(g_{j}, T f_{k}\right)$. In fact, a closer look at this formula shows that it is nothing but the usual formula for the expansion of the determinant of the $n \times n$ matrix $\left(W_{1,1}\left(g_{j} ; f_{k}\right)\right)_{j k}$, i.e.,

$$
\omega_{\beta}\left(a^{*}\left(g_{m}\right) \cdots a^{*}\left(g_{1}\right) a\left(f_{1}\right) \cdots a\left(f_{n}\right)\right)=\delta_{n m} \operatorname{det}\left\{\left(f_{j}, T g_{k}\right)\right\} .
$$

Definition 26. A state $\omega$ on $\mathrm{CAR}(\mathfrak{h})$ or $\mathrm{CAR}^{+}(\mathfrak{h})$ is called gauge-invariant if it is invariant under the gauge group $\vartheta^{t}$ (recall Equ. (13)). It is called gauge-invariant quasi-free if it satisfies

$$
\omega\left(a^{*}\left(g_{m}\right) \cdots a^{*}\left(g_{1}\right) a\left(f_{1}\right) \cdots a\left(f_{n}\right)\right)=\delta_{n m} \operatorname{det}\left\{\left(f_{j}, T g_{k}\right)\right\}
$$

for some self-adjoint operator $T$ on $\mathfrak{h}$ such that $0 \leq T \leq I$, all integers $n, m$ and all $f_{1}, \ldots, f_{n}, g_{1}, \ldots, g_{m} \in \mathfrak{h}$.

Remark 9. It is easy to see that a gauge-invariant quasi-free state is indeed gauge-invariant. One can show that, given an operator $T$ on $\mathfrak{h}$ such that $0 \leq T \leq I$, there exists a unique gauge-invariant quasi-free state such that Equ. (57) holds (see the Notes to Section 2.5.3 in [12] for references).

Thus, we have shown:

Theorem 22. Let $\tau^{t}(a(f))=a\left(\mathrm{e}^{i t h} f\right)$, then the unique $\left(\tau^{t}, \beta\right)$-KMS state of the $C^{*}$-dynamical system $\left(\mathrm{CAR}^{+}(\mathfrak{h}), \tau^{t}\right)$ is the quasi-free gauge invariant state generated by $T=\left(1+\mathrm{e}^{\beta h}\right)^{-1}$.

Example 17. (Quantum spin system, continuation of Example 3) The reader should consult Chapter 6.2 in [12] as well as [38] for more complete discussions.

For any finite subset $\Lambda \subset \Gamma$ and any local observable $A \in \mathfrak{A}_{\text {loc }}$ we set

$$
\omega_{\Lambda}(A) \equiv \frac{\operatorname{Tr}_{\mathfrak{h}_{\Lambda \cup X}}\left(\mathrm{e}^{-\beta H_{\Lambda}} A\right)}{\operatorname{Tr}_{\mathfrak{h}_{\Lambda \cup X}}\left(\mathrm{e}^{-\beta H_{\Lambda}}\right)},
$$

whenever $A \in \mathfrak{A}_{X}$. It is easy to see that $\omega_{\Lambda}(A)$ does not depend on the choice of the finite subset $X$. By continuity, $\omega_{\Lambda}$ extends to a state on $\mathfrak{A}$. We say that a state $\omega$ on $\mathfrak{A}$ is a thermodynamic limit of the net $\omega_{\Lambda}, \Lambda \uparrow \Gamma$, if there exists a subnet $\Lambda_{\alpha}$ such that

$$
\omega(A)=\lim _{\alpha} \omega_{\Lambda_{\alpha}}(A)
$$

for all $A \in \mathfrak{A}$. Since $E(\mathfrak{A})$ is weak- $\star$ compact the set of thermodynamic limits of the net $\omega_{\Lambda}$, is not empty. Let us now prove that any thermodynamic limit of $\omega_{\Lambda}$ is a $(\tau, \beta)$-KMS state.

Following the discussion at the beginning of this subsection we remark that for any finite subset $\Lambda \subset \Gamma$ and any $A, B \in \mathfrak{A}_{\text {loc }}$ the function

$$
F_{\beta, \Lambda}(A, B ; z) \equiv \omega_{\Lambda}\left(A \tau_{\Lambda}^{z}(B)\right)
$$


is entire analytic and satisfies the $\left(\tau_{\Lambda}, \beta\right)$-KMS conditions (52), (53). Since for $t, \eta \in \mathbb{R}$ one has $\left|\omega_{\Lambda}\left(A \tau_{A}^{t+i \eta}(B)\right)\right| \leq\|A\|\left\|\tau^{i \eta}(B)\right\|$, it is also bounded on any horizontal strip $\{t+i \eta \mid t \in \mathbb{R}, a \leq \eta \leq b\}$. The boundary conditions (52), (53) and Hadamard three line theorem further yields the bound

$$
\sup _{z \in \overline{S_{\beta}}}\left|F_{\beta, \Lambda}(A, B ; z)\right| \leq\|A\|\|B\| .
$$

Assume now that $\omega$ is the weak- $\star$ limit of the net $\omega_{\Lambda_{\alpha}}$ and let $A, B \in \mathfrak{A}_{\mathrm{loc}}$. Since for $t \in \mathbb{R}$ one has

$$
\lim _{\alpha}\left\|\tau_{\Lambda_{\alpha}}^{t}(B)-\tau^{t}(B)\right\|=0
$$

it follows that

$$
\begin{aligned}
\lim _{\alpha} F_{\beta, \Lambda_{\alpha}}(A, B ; t) & =\omega\left(A \tau^{t}(B)\right), \\
\lim _{\alpha} F_{\beta, \Lambda_{\alpha}}(A, B ; t+i \beta) & =\omega\left(\tau^{t}(B) A\right) .
\end{aligned}
$$

From the bound (58) and Montel theorem we conclude that some subsequence $F_{\beta, \Lambda_{\alpha_{n}}}(A, B ; z)$ is locally uniformly convergent in the open strip $S_{\beta}$. Let us denote its limit, which is analytic in $S_{\beta}$, by $F_{\beta}(A, B ; z)$.

The estimate (16) and the expansion (18) show that, for $B \in \mathfrak{A}_{\mathrm{loc}}$,

$$
\lim _{\epsilon \rightarrow 0}\left\|\tau_{\Lambda}^{i \epsilon}(B)-B\right\|=0
$$

holds uniformly in $\Lambda$. From the identity

$$
F_{\beta}(A, B ; t+i \epsilon)-\omega\left(A \tau^{t}(B)\right)=\lim _{n} \omega_{\Lambda_{\alpha_{n}}}\left(A\left(\tau_{\Lambda_{\alpha_{n}}}^{t+i \epsilon}(B)-\tau_{\Lambda_{\alpha_{n}}}^{t}(B)\right)\right),
$$

and the bound

$$
\begin{aligned}
\left|\omega_{\Lambda_{\alpha_{n}}}\left(A\left(\tau_{\Lambda_{\alpha_{n}}}^{t+i \epsilon}(B)-\tau_{\Lambda_{\alpha_{n}}}^{t}(B)\right)\right)\right| & =\left|\omega_{\Lambda_{\alpha_{n}}}\left(\tau_{\Lambda_{\alpha_{n}}}^{-t}(A)\left(\tau_{\Lambda_{\alpha_{n}}}^{i \epsilon}(B)-B\right)\right)\right| \\
& \leq\|A\|\left\|\tau_{\Lambda_{\alpha_{n}}}^{i \epsilon}(B)-B\right\|,
\end{aligned}
$$

we thus conclude that

$$
\lim _{\epsilon \downarrow 0} F_{\beta}(A, B ; t+i \epsilon)=\omega\left(A \tau^{t}(B)\right) .
$$

Using the $\left(\tau_{\Lambda}, \beta\right)$-KMS conditions (53) we prove in a completely similar way that

$$
\lim _{\epsilon \downarrow 0} F_{\beta}(A, B ; t+i \beta-i \epsilon)=\omega\left(\tau^{t}(B) A\right),
$$

and we conclude that $F_{\beta}(A, B ; z)$ extends to a continuous function on the closed strip $\overline{S_{\beta}}$. Moreover, this function clearly satisfies the bound

$$
\sup _{z \in \overline{S_{\beta}}}\left|F_{\beta}(A, B ; z)\right| \leq\|A\|\|B\|,
$$


as well as the $(\tau, \beta)$-KMS conditions (52) and (53).

Finally, we note that any $A, B \in \mathfrak{A}$ can be approximated by sequences $A_{n}, B_{n} \in \mathfrak{A}_{\mathrm{loc}}$. As in the proof of Theorem 21 the bound (59) shows that the sequence $F_{\beta}\left(A_{n}, B_{n} ; z\right)$ converges uniformly on $\overline{S_{\beta}}$. Its limit, which we denote by $F_{\beta}(A, B ; z)$ then satisfies all the requirements of Definition 25 .

I conclude this brief introduction to KMS states with the following complement to Proposition 5 which is very useful in many applications to open quantum systems.

Proposition 12. Let $\left(\mathfrak{C}, \tau^{t}, \omega\right)$ be a quantum dynamical system with normal form $\left(\pi_{\omega}, \mathfrak{C}_{\omega}, \mathcal{H}_{\omega}, L_{\omega}, \Omega_{\omega}\right)$. Assume that there exists a *-subalgebra $\mathfrak{D} \subset \mathfrak{C}$ with the following properties.

(i) $\pi_{\omega}(\mathfrak{D})$ is $\sigma$-weakly dense in $\mathfrak{C}_{\omega}$.

(ii) For each $A, B \in \mathfrak{D}$ there exists a function $F_{\beta}(A, B ; z)$ analytic in the strip $S_{\beta}$, continuous and bounded on its closure and satisfying the KMS boundary conditions (52), (53).

Denote by $\hat{\omega}=\left(\Omega_{\omega},(\cdot) \Omega_{\omega}\right)$ the normal extension of $\omega$ to the enveloping von Neumann algebra $\mathfrak{C}_{\omega}$ and by $\hat{\tau}^{t}(A)=\mathrm{e}^{i t L_{\omega}} A \mathrm{e}^{-i t L_{\omega}}$ the $W^{*}$-dynamics induced by $\tau^{t}$ on this algebra. Then the following hold.

(i) $\hat{\omega}$ is $\left(\hat{\tau}^{t}, \beta\right)-K M S$ and faithful.

(ii) $\left(\mathfrak{C}_{\omega}, \hat{\tau}^{t}, \hat{\omega}\right)$ is in standard form. In particular the $\omega$-Liouvillean $L_{\omega}$ coincide with the standard Liouvillean $L$.

(iii) The modular operator of the state $\hat{\omega}$ is given by $\Delta_{\hat{\omega}}=\mathrm{e}^{-\beta L}$.

Proof. Let $A, B \in \mathfrak{C}_{\omega}$ be such that $\|A\| \leq 1$ and $\|B\| \leq 1$. By the Kaplansky density theorem there exists nets $A_{\alpha}, B_{\alpha}$ in $\pi_{\omega}(\mathfrak{D})$ such that $\left\|A_{\alpha}\right\| \leq 1$, $\left\|B_{\alpha}\right\| \leq 1$ and $A_{\alpha} \rightarrow A, B_{\alpha} \rightarrow B$ in the $\sigma$-strong* topology. In particular, if we set

$d_{\alpha} \equiv \max \left(\left\|\left(A_{\alpha}-A\right) \Omega_{\omega}\right\|,\left\|\left(A_{\alpha}^{*}-A^{*}\right) \Omega_{\omega}\right\|,\left\|\left(B_{\alpha}-B\right) \Omega_{\omega}\right\|,\left\|\left(B_{\alpha}^{*}-B^{*}\right) \Omega_{\omega}\right\|\right)$,

then $\lim _{\alpha} d_{\alpha}=0$. By Hypothesis (ii) for each $\alpha$ there exists a function $F_{\alpha}(z)$ analytic in $S_{\beta}$, continuous and bounded on its closure and such that $F_{\alpha}(t)=$ $\hat{\omega}\left(A_{\alpha} \hat{\tau}^{t}\left(B_{\alpha}\right)\right)$ and $F_{\alpha}(t+i \beta)=\hat{\omega}\left(\hat{\tau}^{t}\left(B_{\alpha}\right) A_{\alpha}\right)$ for $t \in \mathbb{R}$. Hence, $F_{\alpha}(z)-F_{\alpha^{\prime}}(z)$ is also analytic in $S_{\beta}$, continuous and bounded on its closure. By the CauchySchwarz inequality we have the bounds

$$
\begin{aligned}
\left|F_{\alpha}(t)-F_{\alpha^{\prime}}(t)\right| & \leq 2\left(d_{\alpha}+d_{\alpha^{\prime}}\right), \\
\left|F_{\alpha}(t+i \beta)-F_{\alpha^{\prime}}(t+i \beta)\right| & \leq 2\left(d_{\alpha}+d_{\alpha^{\prime}}\right),
\end{aligned}
$$

and the Hadamard three line theorem yields

$$
\sup _{z \in \overline{S_{\beta}}}\left|F_{\alpha}(z)-F_{\alpha^{\prime}}(z)\right| \leq 2\left(d_{\alpha}+d_{\alpha^{\prime}}\right) .
$$


Thus, $F_{\alpha}$ is a Cauchy net for the uniform topology. It follows that $\lim _{\alpha} F_{\alpha}=F$ exists, is analytic in $S_{\beta}$ and continuous on its closure. Moreover, we have

$$
\begin{aligned}
F(t) & =\lim _{\alpha} \hat{\omega}\left(A_{\alpha} \hat{\tau}^{t}\left(B_{\alpha}\right)\right)=\lim _{\alpha}\left(A_{\alpha}^{*} \Omega_{\alpha}, \mathrm{e}^{i t L_{\omega}} B_{\alpha} \Omega_{\omega}\right) \\
& =\left(A^{*} \Omega_{\omega}, \mathrm{e}^{i t L_{\omega}} B \Omega_{\omega}\right)=\hat{\omega}\left(A \hat{\tau}^{t}(B)\right)
\end{aligned}
$$

and similarly $F(t+i \beta)=\hat{\omega}\left(\hat{\tau}^{t}(B) A\right)$. This shows that $\hat{\omega}$ is $\left(\hat{\tau}^{t}, \beta\right)$-KMS.

Let now $A \in \mathfrak{C}_{\omega}$ be such that $A \Omega_{\omega}=0$. Then the function $F_{\beta}\left(A^{*}, A ; z\right)$ of Definition 25 satisfies

$$
F_{\beta}\left(A^{*}, A ; t\right)=\hat{\omega}\left(A^{*} \hat{\tau}^{t}(A)\right)=\left(A \Omega_{\omega}, \mathrm{e}^{i t L_{\omega}} A \Omega_{\omega}\right)=0 .
$$

By the Schwarz reflection principle, this function extends to an analytic function on the strip $\{z \in \mathbb{C} \mid-\beta<\operatorname{Im} z<\beta\}$ which vanishes on $\mathbb{R}$. It is therefore identically zero. In particular $F_{\beta}\left(A^{*}, A ; i \beta\right)=\hat{\omega}\left(A A^{*}\right)=\left\|A^{*} \Omega_{\omega}\right\|^{2}=0$. For any $B \in \mathfrak{C}_{\omega}$, the same argument shows that $(B A)^{*} \Omega_{\omega}=A^{*} B^{*} \Omega_{\omega}=0$ and since $\Omega_{\omega}$ is cyclic we can conclude that $A^{*}=0$ and $A=0$. Thus, $\hat{\omega}$ is faithful and (ii) follows from Proposition 5.

Note that by Remark 8 iii the state $\hat{\omega}$ is $\left(\hat{\tau}^{-\beta t},-1\right)$-KMS. (iii) follows from the fact that the modular group $\sigma_{\hat{\omega}}^{t}(A)=\Delta_{\hat{\omega}}^{i t} A \Delta_{\hat{\omega}}^{-i t}$ of a faithful normal state is the unique $W^{*}$-dynamics for which $\omega$ is a $\beta=-1$ KMS state (Takesaki's theorem, see Lecture [7], Theorem 18).

Example 18. (Ideal Bose gas, continuation of Example 10) The characteristic function of the thermal equilibrium state of an ideal Bose gas can be obtained from the explicit calculation of the thermodynamic limit of the unique Gibbs state of a finite Bose gas (see Chapter 1 in [12], see also Subsection 4.4 in $[36])$. We shall get it by assuming that, as in the Fermionic case, the KMS state is quasi-free. Thus, we are looking for a non-negative operator $\rho$ on $\mathfrak{h}$ such that

$$
\omega(W(f))=\mathrm{e}^{-(f,(I+2 \rho) f) / 4},
$$

satisfies the $(\tau, \beta)$-KMS condition. Using the CCR relation (19) we can compute

$$
\omega\left(W(f) \tau^{t}(W(f))\right)=\omega\left(W\left(f+\mathrm{e}^{i t h} f\right)\right) \mathrm{e}^{-i \operatorname{Im}\left(f, \mathrm{e}^{i t h} f\right)}=\mathrm{e}^{-(f, \gamma(t) f) / 4},
$$

where

$$
\gamma(t)=\left(I+\mathrm{e}^{-i t h}\right)(I+2 \rho)\left(I+\mathrm{e}^{i t h}\right)+2 i \sin t h .
$$

Assuming that $f$ is an entire analytic vector for the group $\mathrm{e}^{i t h}$, analytic continuation to $t=i \beta$ yields

$$
\gamma(i \beta)=\left(I+\mathrm{e}^{\beta h}\right)(I+2 \rho)\left(I+\mathrm{e}^{-\beta h}\right)-2 \operatorname{sh} \beta h,
$$

and the KMS condition (54) requires that $\gamma(i \beta)=\gamma(0)$. This equation is easily solved for $\rho$ and its solution is given by Planck's black-body radiation law 


$$
\rho=\frac{1}{\mathrm{e}^{\beta h}-1}
$$

It is clear from the singularity at $h=0$ in Equ. (61) that if $0 \in \sigma(h)$ one can not hope to get a state on $\operatorname{CCR}(\mathcal{D})$ without further assumption on $\mathcal{D}$.

Assume that $h>0$, i.e., that 0 is not an eigenvalue of $h$ and that $\mathcal{D}=\mathrm{D}\left(h^{-1 / 2}\right)$. Then according to Definition 17, Equ. (60), (61) define a quasi-free, $\tau^{t}$-invariant state $\omega$ on $\operatorname{CCR}(\mathcal{D})$. Let $\left(\operatorname{CCR}(\mathcal{D}), \tau^{t}, \omega\right)$ be the associated quantum dynamical system and $\left(\pi_{\omega}, \mathfrak{M}_{\omega}, \mathcal{H}_{\omega}, L_{\omega}, \Omega_{\omega}\right)$ its normal form as described in Example 10. Note that since finite linear combinations of elements of $W(\mathcal{D})$ are dense in $\operatorname{CCR}(\mathcal{D})$ one has $\mathfrak{M}_{\omega}^{\prime}=\pi_{\omega}(W(\mathcal{D}))^{\prime}$ and $\mathfrak{M}_{\omega}=\pi_{\omega}(W(\mathcal{D}))^{\prime \prime}$.

$\mathcal{D}$ equipped with the scalar product $(f, g)_{\mathcal{D}} \equiv(f,(I+2 \rho) g)$ is a Hilbert space. Since $\mathrm{e}^{i t h}$ is a strongly continuous unitary group on $\mathcal{D}$ the subspace $\mathcal{A} \subset \mathcal{D}$ of entire analytic vectors for $\mathrm{e}^{i t h}$ is dense. It follows that any $f \in \mathcal{D}$ can be approximated in the norm of $\mathcal{D}$ by a sequence $f_{n} \in \mathcal{A}$.

A simple calculation using the CCR shows that for $f, f^{\prime}, g \in \mathcal{D}$,

$$
\left\|\left(\pi_{\omega}(W(f))-\pi_{\omega}\left(W\left(f^{\prime}\right)\right)\right) \pi_{\omega}(W(g)) \Omega_{\omega}\right\|^{2}=2\left(1-\mathrm{e}^{-\left\|f-f^{\prime}\right\|_{\mathcal{D}}^{2} / 4} \cos \theta\right),
$$

where $\theta \equiv \operatorname{Im}\left(f-f^{\prime}, g\right)+\operatorname{Im}\left(f, f^{\prime}\right) / 2$. Since the elements of $\pi_{\omega}(W(\mathcal{D}))$ are unitary and $\Omega_{\omega}$ is cyclic it follows that

$$
\mathrm{s}-\lim _{n} \pi_{\omega}\left(W\left(f_{n}\right)\right)=\pi_{\omega}(W(f)),
$$

whenever $f_{n}$ converges to $f$ in $\mathcal{D}$. We conclude that

$$
\pi_{\omega}(W(\mathcal{A}))^{\prime}=\pi_{\omega}(W(\mathcal{D}))^{\prime}=\mathfrak{M}_{\omega}^{\prime},
$$

and hence $\pi_{\omega}(W(\mathcal{A}))^{\prime \prime}=\mathfrak{M}_{\omega}$.

Denote by $\mathfrak{D} \subset \operatorname{CCR}(\mathcal{D})$ the linear span of $W(\mathcal{A})$. By the von Neumann density theorem $\pi_{\omega}(\mathfrak{D})$ is $\sigma$-weakly dense in $\mathfrak{M}_{\omega}$. Moreover, an explicit calculation shows that $\omega\left(W(f) \tau^{t}(W(g))\right)=\mathrm{e}^{-\phi_{t}(f, g) / 4}$ where

$$
\phi_{t}(f, g)=\|f\|_{\mathcal{D}}^{2}+\|g\|_{\mathcal{D}}^{2}+2\left(g, \frac{\mathrm{e}^{-i t h}}{I+\mathrm{e}^{\beta h}} f\right)_{\mathcal{D}}+2\left(f, \frac{\mathrm{e}^{i t h}}{I+\mathrm{e}^{-\beta h}} g\right)_{\mathcal{D}} .
$$

If $f, g \in \mathcal{A}$ then $\phi_{t}(f, g)$ is an entire analytic function of $t$ and $\phi_{t+i \beta}(f, g)=$ $\phi_{t}(g, f)$. Thus, $\mathfrak{D}$ fulfills the requirements of Proposition 12. It follows that $\hat{\omega}(A)=\left(\Omega_{\omega}, A \Omega_{\omega}\right)$ is a $\beta$-KMS state for the $W^{*}$-dynamics generated by the Liouvillean $L=L_{\omega}$. Note that since $\operatorname{Ker} \rho=\{0\}$ the GNS representation associated to $\omega$ is given by

$$
\begin{aligned}
\mathcal{H}_{\omega} & =\mathcal{L}^{2}\left(\Gamma_{+}(\mathfrak{h})\right), \\
\pi_{\omega}(W(f)) X & =W\left((I+\rho)^{1 / 2} f\right) X W\left(\rho^{1 / 2} f\right)^{*}, \\
\Omega_{\omega} & =\Omega(\Omega, \cdot),
\end{aligned}
$$


where $\Omega$ is the Fock vacuum in $\Gamma_{+}(\mathfrak{h})$. The Liouvillean $L$ is given by

$$
\mathrm{e}^{i t L} X=\Gamma\left(\mathrm{e}^{i t h}\right) X \Gamma\left(\mathrm{e}^{-i t h}\right),
$$

Moreover, since $\hat{\omega}$ is faithful we have $\left[\mathfrak{M}_{\omega}^{\prime} \Omega_{\omega}\right]=I$ and Corollary 3 applies.

Exercise 26. Show that the standard form of $\mathfrak{M}_{\omega}$ on $\mathcal{H}_{\omega}$ is specified by conjugation $J: X \mapsto X^{*}$ and the cone $\mathcal{C}=\left\{X \in \mathcal{L}^{2}\left(\Gamma_{+}(\mathfrak{h})\right) \mid X \geq 0\right\}$.

Exercise 27. Show that if $h$ has purely absolutely continuous spectrum then 0 is a simple eigenvalue of $L$ with eigenvector $\Omega_{\omega}$. Show that the spectrum of $L$ on $\left\{\Omega_{\omega}\right\}^{\perp}$ is purely absolutely continuous. Conclude that the quantum dynamical system $\left(\operatorname{CCR}(\mathcal{D}), \tau^{t}, \omega\right)$ is mixing. What happens if $h$ has nonempty singular spectrum?

The thermodynamics of the ideal Bose gas is more complex than the above picture. In fact, due to the well known phenomenon of Bose-Einstein condensation, non-unique KMS states are possible. I refer to Lecture [36] and [12] for a detailed discussion.

\subsection{Perturbation Theory of KMS States}

Consider the finite dimensional $C^{*}$-dynamical system defined by $\mathfrak{A}=\mathfrak{B}\left(\mathbb{C}^{n}\right)$ and $\tau^{t}(X)=\mathrm{e}^{i t H} X \mathrm{e}^{-i t H}$ for some self-adjoint matrix $H$. Then

$$
\omega(X)=\frac{\operatorname{Tr}\left(\mathrm{e}^{-\beta H} X\right)}{\operatorname{Tr}\left(\mathrm{e}^{-\beta H}\right)}=\frac{\operatorname{Tr}\left(\mathrm{e}^{-\beta H / 2} X \mathrm{e}^{-\beta H / 2}\right)}{\operatorname{Tr}\left(\mathrm{e}^{-\beta H}\right)},
$$

is the unique $\left(\tau^{t}, \beta\right)$-KMS state. If $V$ is another self-adjoint matrix then the perturbed dynamics $\tau_{V}^{t}$ as well as the perturbed KMS state $\omega_{V}$ are obtained by replacing $H$ by $H+V$. Note that, in the present situation, the definition (45) of the unitary cocycle $\Gamma_{V}^{t}$ reads

$$
\Gamma_{V}^{t}=\mathrm{e}^{i t(H+V)} \mathrm{e}^{-i t H},
$$

which is obviously an entire function of $t$. Thus, we can express $\omega_{V}$ in terms of $\omega$ as

$$
\omega_{V}(X)=\frac{\omega\left(X \Gamma_{V}^{i \beta}\right)}{\omega\left(\Gamma_{V}^{i \beta}\right)}=\frac{\omega\left(\Gamma_{V}^{i \beta / 2 *} X \Gamma_{V}^{i \beta / 2}\right)}{\omega\left(\Gamma_{V}^{i \beta / 2 *} \Gamma_{V}^{i \beta / 2}\right)} .
$$

Let $\left(\mathcal{H}_{\omega}, \pi_{\omega}, \Omega_{\omega}\right)$ be the cyclic representation of $\omega$ and $L$ the Liouvillean ( $\omega$ Liouvillean and standard Liouvillean coincide since $\omega$ is faithful). Then on has

$$
\pi_{\omega}\left(\Gamma_{V}^{i \beta / 2}\right) \Omega_{\omega}=\pi_{\omega}\left(\Gamma_{V}^{i \beta / 2}\right) \mathrm{e}^{-\beta L / 2} \Omega_{\omega}=\mathrm{e}^{-\beta\left(L+\pi_{\omega}(V)\right) / 2} \Omega_{\omega},
$$

by Equ. (48). Thus we can write Equ. (62) as

$$
\omega_{V}(X)=\frac{\left(\Omega_{\omega_{V}}, \pi_{\omega}(X) \Omega_{\omega_{V}}\right)}{\left(\Omega_{\omega_{V}}, \Omega_{\omega_{V}}\right)},
$$


where $\Omega_{\omega_{V}}=\mathrm{e}^{-\beta\left(L+\pi_{\omega}(V)\right) / 2} \Omega_{\omega}$. The cocycle property (47) further gives

$$
\Gamma_{V}^{i \beta / 2}=\Gamma_{V}^{i \beta / 4} \tau^{i \beta / 4}\left(\Gamma_{V}^{i \beta / 4}\right)=\Gamma_{V}^{i \beta / 4} \tau^{i \beta / 2}\left(\tau^{i \beta / 4}\left(\Gamma_{V}^{i \beta / 4}\right)\right),
$$

and $\tau^{-i \beta / 4}\left(\Gamma_{V}^{i \beta / 4}\right)=\left(\Gamma_{V}^{-i \beta / 4}\right)^{-1}$. Since $\Gamma_{V}^{\bar{z} *}$ is analytic and equals $\left(\Gamma_{V}^{z}\right)^{-1}$ for real $z$, they are equal for all $z$ and

$$
\Gamma_{V}^{i \beta / 2}=\Gamma_{V}^{i \beta / 4} \tau^{i \beta / 2}\left(\Gamma_{V}^{i \beta / 4 *}\right) .
$$

We can rewrite the perturbed vector $\Omega_{\omega_{V}}$ as

$\Omega_{\omega_{V}}=\pi_{\omega}\left(\Gamma_{V}^{i \beta / 4}\right) \mathrm{e}^{-\beta L / 2} \pi_{\omega}\left(\Gamma_{V}^{i \beta / 4}\right)^{*} \Omega_{\omega}=\pi_{\omega}\left(\Gamma_{V}^{i \beta / 4}\right) \mathrm{e}^{-\beta L / 2} J \Delta_{\omega}^{1 / 2} \pi_{\omega}\left(\Gamma_{V}^{i \beta / 4}\right) \Omega_{\omega}$,

and since $J \Delta_{\omega}^{1 / 2}=J \mathrm{e}^{-\beta L / 2}=\mathrm{e}^{\beta L / 2} J$ we conclude that

$$
\Omega_{\omega_{V}}=\pi_{\omega}\left(\Gamma_{V}^{i \beta / 4}\right) J \pi_{\omega}\left(\Gamma_{V}^{i \beta / 4}\right) \Omega_{\omega} \in \mathcal{C} .
$$

Thus $\Omega_{\omega_{V}}$ is, up to normalization, the unique standard vector representative of the perturbed KMS-state $\omega_{V}$.

The main difficulty in extending this formula to more general situations is to show that $\Omega_{\omega} \in \mathrm{D}\left(\mathrm{e}^{-\beta\left(L+\pi_{\omega}(V)\right) / 2}\right)$. Indeed, even if $V$ is bounded, the Liouvillean $L$ is usually unbounded below and ordinary perturbation theory of quasi-bounded semi-groups fails. If $V$ is such that $\tau^{t}(V)$ is entire analytic, this can be done using (63) since the cocycle $\Gamma_{V}^{t}$ is then analytic, as the solution of a linear differential equation with analytic coefficients. It is possible to extend the result to general bounded perturbations using an approximation argument.

Theorem 23. Let $\left(\mathfrak{C}, \tau^{t}\right)$ be a $C^{*}$ - or $W^{*}$-dynamical system and $V \in \mathfrak{C}$ a local perturbation. There exists a bijective map $\omega \mapsto \omega_{V}$ between the set of $\left(\tau^{t}, \beta\right)$ $K M S$ states and the set of $\left(\tau_{V}^{t}, \beta\right)-K M S$ states on $\mathfrak{C}$ such that $\omega_{V} \in N(\mathfrak{C}, \omega)$ and $\left(\omega_{V_{1}}\right)_{V_{2}}=\omega_{V_{1}+V_{2}}$.

Let $\omega$ be a $\left(\tau^{t}, \beta\right)$-KMS state on $\mathfrak{C}$. Denote by $L$ the (standard) Liouvillean of the quantum dynamical system $\left(\mathfrak{C}_{\omega}, \hat{\tau}^{t}, \hat{\omega}\right)$ and by $\Omega \in \mathcal{H}_{\omega}$ the standard vector representative of $\hat{\omega}$. For any local perturbation $V \in \mathfrak{C}_{\omega}$ one has:

(i) $\Omega \in \mathrm{D}\left(\mathrm{e}^{-\beta(L+V) / 2}\right)$.

(ii) Up to normalization, $\Omega_{V}=\mathrm{e}^{-\beta(L+V) / 2} \Omega$ is the standard vector representative of $\hat{\omega}_{V}$.

(iii) $\Omega_{V}$ is cyclic and separating for $\mathfrak{C}_{\omega}$.

(iv) For any $V_{1}, V_{2} \in \mathfrak{C}_{\omega}$ one has $\left(\Omega_{V_{1}}\right)_{V_{2}}=\Omega_{V_{1}+V_{2}}$.

(v) The Peierls-Bogoliubov inequality holds: $\mathrm{e}^{-\beta \hat{\omega}(V)} \leqslant\left\|\Omega_{V}\right\|^{2}$.

(vi) The Golden-Thompson inequality holds: $\left\|\Omega_{V}\right\|^{2} \leqslant \hat{\omega}\left(\mathrm{e}^{-\beta V}\right)$.

(vii) If $V_{n} \in \mathfrak{C}_{\omega}$ strongly converges to $V \in \mathfrak{C}_{\omega}$ then $\Omega_{V_{n}}$ converges in norm to $\Omega_{V}$ and $\omega_{V_{n}}$ converges in norm to $\omega_{V}$.

In the case of unbounded perturbations, one can use an approximation by bounded perturbations to obtain 
Theorem 24. Let $\left(\mathfrak{M}, \tau^{t}, \omega\right)$ be a quantum dynamical system and suppose that $\omega$ is a $\left(\tau^{t}, \beta\right)-K M S$ state. Assume that the system is in standard form $(\mathfrak{M}, \mathcal{H}, J, \mathcal{C})$. Denote by $L$ its standard Liouvillean and by $\Omega$ the standard vector representative of $\omega$. Let $V$ be a self-adjoint operator affiliated to $\mathfrak{M}$ and such that the following conditions are satisfied:

(i) $L+V$ is essentially self-adjoint on $\mathrm{D}(L) \cap \mathrm{D}(V)$.

(ii) $L+V-J V J$ is essentially self-adjoint on $\mathrm{D}(L) \cap \mathrm{D}(V) \cap \mathrm{D}(J V J)$.

(iii) $\Omega \in \mathrm{D}\left(\mathrm{e}^{-\beta V / 2}\right)$.

Then the following conclusions hold:

(i) $\Omega \in \mathrm{D}\left(\mathrm{e}^{-\beta(L+V) / 2}\right)$.

(ii) Up to normalization, $\Omega_{V}=\mathrm{e}^{-\beta(L+V) / 2} \Omega$ is the standard vector representative of a $\left(\tau_{V}^{t}, \beta\right)-K M S$ state $\omega_{V}$.

(iii) $\Omega_{V}$ is cyclic and separating for $\mathfrak{M}$.

(iv) The Peierls-Bogoliubov inequality holds: $\mathrm{e}^{-\beta \hat{\omega}(V)} \leqslant\left\|\Omega_{V}\right\|^{2}$.

(v) The Golden-Thompson inequality holds: $\left\|\Omega_{V}\right\|^{2} \leqslant \hat{\omega}\left(\mathrm{e}^{-\beta V}\right)$.

(vi) For any $\lambda \in[0,1]$ the operator $\lambda V$ satisfies the hypotheses (i), (ii) and (iii) and one has $\lim _{\lambda \downarrow 0}\left\|\Omega_{\lambda V}-\Omega\right\|=0$ and $\lim _{\lambda \downarrow 0}\left\|\omega_{\lambda V}-\omega\right\|=0$.

\section{References}

1. Araki, H., Woods, E.J.: Representations of the canonical commutation relations describing a nonrelativistic infinite free Bose gas. J. Math. Phys. 4 (1963), 637.

2. Araki, H.: Relative entropy of states on von Neumann algebras. Publ. Res. Inst. Math. Sci. Kyoto Univ. 11 (1975), 809.

3. Araki, H.: Positive cone, Radon-Nikodym theorems, relative Hamiltonian and the Gibbs condition in statistical mechanics. In $C^{*}$-Algebras and their Applications to Statistical Mechanics and Quantum Field Theory. D. Kastler editor. North-Holand, Amsterdam, 1976.

4. Araki, H.: Relative entropy of states on von Neumann algebras II. Publ. Res. Inst. Math. Sci. Kyoto Univ. 13 (1977), 173.

5. Arnold, V.I., Avez, A.: Ergodic Problems of Classical Mechanics. Benjamin, New York, 1968.

6. Aschbacher, W., Jakšić, V., Pautrat, Y., Pillet, C.-A.: Topics in nonequilibrium quantum statistical mechanics. Volume 3 in this series.

7. Attal, S.: Elements of operator algebras. Part 3 in this volume.

8. Bach, V., Fröhlich, J., Sigal, I.M.: Return to equilibrium. J. Math. Phys. 41 (2000), 3985.

9. Benatti, F.: Deterministic Chaos in Infinite Quantum Systems. Springer, Berlin, 1993.

10. Birkhoff, G.D.: Proof of the ergodic theorem. Proc. Nat. Acad. Sci. (U.S.A.) 17 (1931), 656.

11. Bratteli, O., Robinson, D.W.: Operator Algebras and Quantum Statistical Mechanics I. Springer, Berlin, 1979. 
12. Bratteli, O., Robinson, D.W.: Operator Algebras and Quantum Statistical Mechanics II. Springer, Berlin, 1996.

13. Cohen-Tanoudji, C., Diu, B., Laloe, F.: Quantum Mechanics. Wiley, New York, 1977.

14. Connes, A.: Une classification des facteurs de type III. Ann. Sci. Ecole Norm. Sup. 6 (1973), 133.

15. Cornfeld, I.P., Fomin, S.V., Sinai, Ya G.: Ergodic Theory. Springer, Berlin, 1982.

16. Dereziński, J., Jakšić, V.: Return to equilibrium for Pauli-Fierz systems. Ann. H. Poincaré 4 (2003), 739.

17. Dereziński, J., Jakšić, V., Pillet, C.-A.: Perturbation theory of $W^{*}$ dynamics, Liouvilleans and KMS-states. Rev. Math. Phys. 15 (2003), 447.

18. Dereziński, J.: Introduction to representations of canonical commutation and anticommutation relations. In the lecture notes of the summer school "Large Coulomb Systems-QED", held in Nordfjordeid, August 11-18 2004. To be published in Lecture Notes in Mathematics.

19. Donald, M.J.: Relative Hamiltonians which are not bounded from above. J. Funct. Anal. 91 (1990), 143.

20. Ford, G.W., Kac, M., Mazur, P.: Statistical mechanics of assemblies of coupled oscillators. J. Math. Phys. 6 (1965), 504.

21. Fröhlich, J., Merkli, M.: Thermal ionization. Math. Phys., Analysis and Geometry 7 (2004), 239.

22. Fröhlich, J., Merkli, M.: Another return of "Return to Equilibrium". Commun. Math. Phys. 251 (2004), 235.

23. Haagerup, U.: The standard form of von Neumann algebras. Math. Scand. 37 (1975), 271.

24. Jadczyk, A.Z.: On some groups of automorphisms of von Neumann algebras with cyclic and separating vector. Commun. Math. Phys. 13 (1969), 142.

25. Jakšić, V.: Topics in spectral theory. Part 6 in this volume.

26. Jakšić, V., Kritchevski, E., Pillet, C.-A.: Mathematical theory of the Wigner-Weisskopf atom. In the lecture notes of the summer school "Large Coulomb Systems-QED", held in Nordfjordeid, August 11-18 2004. To be published in Lecture Notes in Mathematics.

27. Jakšić, V., Pillet, C.-A.: On a Model for Quantum Friction III. Ergodic Properties of the Spin-Boson System. Commun. Math. Phys. 178 (1996), 627.

28. Jakšić, V., Pillet, C.-A.: Ergodic properties of classical dissipative systems I. Acta Math. 181 (1998), 245.

29. Jakšić, V., Pillet, C.-A.: Non-equilibrium steady states of finite quantum systems coupled to thermal reservoirs. Commun. Math. Phys. 226 (2002), 131.

30. Jakšić, V., Pillet, C.-A.: Mathematical theory of non-equilibrium quantum statistical mechanics. J. Stat. Phys. 108 (2002), 787.

31. Jakšić, V., Pillet, C.-A.: A note on eigenvalues of Liouvilleans. J. Stat. Phys. 105 (2001), 937.

32. Joye, A.: Introduction to the theory of linear operators. Part 1 in this volume.

33. Joye, A.: Introduction to quantum statistical mechanics. Part 2 in this volume. 
34. Koopman, B.O.: Hamiltonian systems and transformations in Hilbert spaces. Proc. Nat. Acad. Sci. (U.S.A.) 17 (1931), 315.

35. Mané, R.: Ergodic Theory and Differentiable Dynamics. Springer, Berlin, 1987.

36. Merkli, M.: The ideal quantum gas. Part 5 in this volume.

37. Rey-Bellet, L.: Classical open systems. Volume 2 in this series.

38. Ruelle, D.:Statistical Mechanics. Rigorous Results. Benjamin, New York, 1969.

39. Ruelle, D.: Smooth dynamics and new theoretical ideas in nonequilibrium statistical mechanics. J. Stat. Phys. 95 (1999), 393.

40. Ruelle, D.: Natural nonequilibrium states in quantum statistical mechanics. J. Stat. Phys. 98 (2000), 57.

41. Reed, M., Simon, B.: Methods of Modern Mathematical Physics I: Functional Analysis. Academic Press, New York, 1972.

42. Sakai, S.: $C^{*}$-Algebras and $W^{*}$-Algebras. Springer, Berlin, 1971.

43. Sakai, S.: Perturbations of KMS states in $C^{*}$-dynamical systems. Contemp. Math. 62 (1987), 187.

44. Sakai, S.: Operator Algebras in Dynamical Systems. Cambridge University Press, Cambridge, 1991.

45. Stratila, S., Zsido, L.: Lectures on von Neumann Algebras. Abacus Press, Tunbridge Wells, 1979.

46. Takesaki, M.: Theory of Operator Algebras II. Springer, Berlin, 2003.

47. Thirring, W.: Quantum Mechanics of Large Systems. Springer, Berlin, 1980.

48. von Neumann, J: Proof of the quasiergodic hypothesis. Proc. Nat. Acad. Sci. (U.S.A.) 17 (1932), 70.

49. Walters, P.: An Introduction to Ergodic Theory. Springer, Berlin, 1981. 